\title{
Online-Befragung „Offenes öffentliches Beschaffungswesen“
}

Ziel der empirischen Studie im Rahmen dieser Dissertationsschrift ist die Bewertung der Möglichkeiten und Grenzen einer Öffnung von öffentlichen Einkaufsdaten. Die Sichtweisen aus der Verwaltungs- und Wirtschaftsinformatik, den Wirtschafts-, Rechts-, Sozial- und Politikwissenschaften sollen interdisziplinär berücksichtigt werden. Im Ergebnis soll ein Leitbild zur Öffnung der öffentlichen Einkaufsdaten durch die Verwaltung unter Berücksichtigung organisatorischer, rechtlicher, (gesellschafts-)politischer und technischer Rahmenbedingungen bereitgestellt werden.

\subsection{Design der Befragung}

\subsubsection{Methodik}

Zur Datenerhebung wurde auf eine Online-Befragung zurückgegriffen, weil sie die Möglichkeit bietet, einen Großteil der Antworten in quantitativ verarbeitbarer Form bei einem relativ überschaubaren Zeiteinsatz zu erhalten. Als vorteilhaft können zudem die geringeren Kosten genannt werden, da die Daten digital erhoben und gespeichert werden. Für die Befragten ergibt sich ein geringerer Zeitaufwand, da sie den Zeitpunkt für das Ausfüllen selbst flexibel bestimmen können. Für den Erhebenden sind die Daten sofort digital abruf- und auswertbar. Das bedeutet, dass Zwischenergebnisse zeitnah bereitstehen und während der Erhebung Korrekturen möglich sind. Mit der Online-Erhebung ist eine hohe Reichweite möglich, da die Befragten unabhängig von Zeit und Aufenthaltsort teilnehmen können. Weiters ist die Beeinflussung durch einen Interviewer nicht möglich.

Allerdings gibt es auch Nachteile dieser Methode. Durch die anonyme Befragungssituation besteht die Gefahr, dass die Teilnehmer nicht seriös 
antworten und Ergebnisse verzerrt werden könnten. Weiters besteht das Risiko, dass der Fragebogen nicht vollständig ausgefüllt wird. Dies erschwert die spätere Auswertung beziehungsweise kann die Anzahl auswertbarer Fragebögen reduzieren. Es besteht die Möglichkeit der Verzerrung der Ergebnisse. Es kann nicht abgesichert werden, wer in Person letztlich den Fragebogen ausgefüllt hat; obwohl individualisierte Links verschickt werden, kann dieser weitergeleitet und von einer anderen Person als der eingeladenen einmalig genutzt werden. Gelegenheit zu Rückfragen gibt es in beiden Richtungen nicht, es besteht ein gewisser Interpretationsspielraum. Den Risiken wurde entgegengewirkt, indem die Teilnehmer selektiert wurden (siehe Abschnitt 8.1.4) und die Antwortmöglichkeiten den Fragen so zugeordnet wurden, dass nur dort ein Interpretationsspielraum möglich wurde, wo dieser gewünscht oder unkritisch für das Gesamtergebnis war, wie das folgende Kapitel erläutert.

\subsubsection{Aufbau und Strukturierung des Fragebogens}

Wichtige Vorarbeiten für die Gestaltung des Fragebogens waren die eigene Literaturrecherche und die in den Kapiteln 4 bis 7 angestellten Überlegungen, Recherchen im Umfeld relevanter Konferenzen und Veranstaltungen in den Jahren 2014 bis 2018, im Vorfeld durchgeführte Hintergrundgespräche mit Wissenschaftlern und Praktikern und Feedback aus dem Pretest. Aus den Vorarbeiten entstanden letztlich 40 Fragen, die sich gemäß Tabelle 8.1 auf acht Themenkomplexe verteilten.

Tabelle 8.1 Aufteilung der Fragen auf Themenkomplexe der Studie

\begin{tabular}{l|c|c}
\hline Themenkomplex & Anzahl der Fragen & Davon ,Muss“-Fragen \\
\hline Allgemeine Fragen (AG) & 8 & 5 \\
\hline Organisation (OG) & 3 & 3 \\
\hline Technik (TE) & 11 & 11 \\
\hline Transparenz (TR) & 4 & 4 \\
\hline Partizipation (PA) & 1 & 1 \\
\hline Kollaboration (KO) & 1 & 1 \\
\hline Rechtsprechung (RE) & 2 & 2 \\
\hline Strategie (ST) & 10 & 10 \\
\hline Summe & $\mathbf{4 0}$ & $\mathbf{3 7}$ \\
\hline
\end{tabular}


Von den insgesamt 40 Fragen waren alle bis auf drei allgemeine Fragen verpflichtend zu beantworten. Um einen Ausfüllzwang zu vermeiden, war allerdings die Antwortoption ,keine Antwort“ bei allen Fragen als Standardantwortmöglichkeit enthalten. Die meisten Fragen stammen aus dem Bereich der Technik (11 Fragen) und Strategie (10 Fragen), gefolgt von allgemeinen Fragen (8 Fragen). Der vollständige Fragebogen findet sich im Anhang (siehe Fragebogen A.13). Als Antworttypen wurden die Auswahl, Mehrfachauswahl, der Freitext, die Rangordnung, der Schieberegler und die Skala verwendet. Der Fragebogen orientierte sich in seiner Abfolge an folgenden Abschnitten:

1) Ausgangssituation (Ist-Situation der Öffnung öffentlicher Einkaufsdaten)

2) Zukunft (Soll-Situation der Öffnung öffentlicher Einkaufsdaten)

3) Rand- und Rahmenbedingungen

4) Feedback zum Fragebogen

Die Fragen der Themenkomplexe wurden wie in folgenden Tabellen (Tabelle 8.2, Tabelle 8.3, Tabelle 8.4, Tabelle 8.5) dargestellt auf die jeweiligen Abschnitte des Fragebogens aufgeteilt.

1. Ausgangssituation

Tabelle 8.2 Zuordnung der Fragen auf den Abschnitt Ausgangssituation

\begin{tabular}{l|c|l|l}
\hline Code & Nr. & Frage & Antworttyp \\
\hline AG01 & 1 & $\begin{array}{l}\text { Wie umfangreich ist Ihre Erfahrung im öffentlichen } \\
\text { Einkauf? }\end{array}$ & Auswahl \\
\hline AG02 & 2 & Zu welcher Gruppe zählen Sie sich? & Auswahl \\
\hline AG03 & 3 & $\begin{array}{l}\text { Inwieweit beschäftigen Sie sich bereits mit } \\
\text { „Offenen Verwaltungsdaten“ beziehungsweise } \\
\text { „Open Government Data“? }\end{array}$ & Auswahl \\
\hline ST02 & 4 & $\begin{array}{l}\text { Gibt es bei Ihnen in Bezug auf die Öffnung } \\
\text { der öffentlichen Einkaufsdaten bereits Ansätze, } \\
\text { Initiativen oder Programme, um dies zu fördern/zu } \\
\text { nutzen? }\end{array}$ & Auswahl \\
\hline ST01 & 5 & $\begin{array}{l}\text { Können Sie sich vorstellen, dass die Öffnung } \\
\text { offentlicher Einkaufsdaten als Teil „,Offener Ver- } \\
\text { waltungsdaten“ Vorteile für Ihre Organisation bieten } \\
\text { kann? }\end{array}$ & Mehrfachauswahl \\
\hline
\end{tabular}


Tabelle 8.2 (Fortsetzung)

\begin{tabular}{l|c|l|l}
\hline Code & Nr. & Frage & Antworttyp \\
\hline TE09 & 6 & $\begin{array}{l}\text { Welche öffentlichen Einkaufsdaten kennen Sie und } \\
\text { wie werden diese Ihrer Erfahrung nach heute ver- } \\
\text { offentlicht? }\end{array}$ & Skala mit 3 Stufen \\
\hline TE04 & 7 & $\begin{array}{l}\text { Wie werden öffentliche Einkaufsdaten Ihrer } \\
\text { Erfahrung nach heute überwiegend in den Ver- } \\
\text { waltungen gespeichert? }\end{array}$ & Mehrfachauswahl \\
\hline TE03 & 8 & $\begin{array}{l}\text { Sofern eine elektronische Speicherung der Daten } \\
\text { bereits erfolgt: In welchen Formaten werden } \\
\text { offentliche Einkaufsdaten überwiegend } \\
\text { gespeichert? }\end{array}$ & Mehrfachauswahl \\
\hline TE10 & 9 & $\begin{array}{l}\text { Wo werden die von Ihnen benannten Einkaufsdaten } \\
\text { heute veröffentlicht? }\end{array}$ & Offene Nennungen \\
\hline TE07 & 10 & $\begin{array}{l}\text { Welche Anwendungen (unter anderem } \\
\text { Datenbanken, Software, Apps), Berichte und } \\
\text { Visualisierungen, die auf öffentlichen } \\
\text { Einkaufsdaten beruhen, kennen Sie bereits? } \\
\text { Was behindert aktuell die Freigabe öffentlicher } \\
\text { Einkaufsdaten am meisten? }\end{array}$ & $\begin{array}{l}\text { Rangordnung mit 3 } \\
\text { Rangplätzen }\end{array}$ \\
\hline ST08 & 11 & $\begin{array}{l}\text { Welche wesentlichen Voraussetzungen müssten } \\
\text { noch geschaffen werden, um die Öffnung } \\
\text { offentlicher Einkaufsdaten voranzutreiben? }\end{array}$ & $\begin{array}{l}\text { Rangordnung mit 3 } \\
\text { Rangplätzen }\end{array}$ \\
\hline ST07 & 12 & $\begin{array}{l}\text { Offenen } \\
\text { Wangen }\end{array}$ \\
\hline
\end{tabular}

2. Zukunft

Tabelle 8.3 Zuordnung der Fragen auf den Abschnitt Zukunft

\begin{tabular}{l|l|l|l}
\hline Code & Nr. & Frage & Antworttyp \\
\hline TE12 & 13 & $\begin{array}{l}\text { Inwieweit stimmen Sie einer Veröffentlichung } \\
\text { folgender öffentlicher Einkaufsdaten zu? }\end{array}$ & $\begin{array}{l}\text { Schieberegler mit } \\
11 \text { Zwischenwerten } \\
(0 \%-100 \%)\end{array}$ \\
\hline TE13 & 14 & $\begin{array}{l}\text { Wer sollte aus Ihrer Sicht zukünftig Zugriff auf } \\
\text { welche veröffentlichten öffentlichen Einkaufsdaten } \\
\text { haben? }\end{array}$ & Mehrfachauswahl \\
\hline TR07 & 15 & $\begin{array}{l}\text { Für welche Art der Projekte sollte die Öffnung } \\
\text { der öffentlichen Einkaufsdaten mehr oder weniger } \\
\text { ermöglicht beziehungsweise eingeschränkt werden? }\end{array}$ & $\begin{array}{l}\text { Schieberegler mit } \\
11 \text { Zwischenwerten } \\
(0 \%-100 \%)\end{array}$ \\
\hline TE06 & 16 & $\begin{array}{l}\text { Gibt es aus Ihrer Sicht Warengruppen oder Sektoren, } \\
\text { deren Daten nicht veröffentlicht werden sollten? } \\
\text { Wenn ja, welche und warum? }\end{array}$ & Auswahl \\
\hline
\end{tabular}


Tabelle 8.3 (Fortsetzung)

\begin{tabular}{|c|c|c|c|}
\hline Code & Nr. & Frage & Antworttyp \\
\hline TE11 & 17 & $\begin{array}{l}\text { Wo sollten die von Ihnen benannten öffentlichen } \\
\text { Einkaufsdaten künftig veröffentlicht werden? }\end{array}$ & Offene Nennungen \\
\hline TE20 & 18 & $\begin{array}{l}\text { Welche wirtschaftlichen Konsequenzen hätte die } \\
\text { Bereitstellung offener öffentlicher Einkaufsdaten } \\
\text { über den govdata.de-Verbund? }\end{array}$ & Mehrfachauswahl \\
\hline TE08 & 19 & $\begin{array}{l}\text { Welche Anwendungen, Berichte oder } \\
\text { Visualisierungen wären zukünftig aus Ihrer Sicht } \\
\text { sinnvoll? }\end{array}$ & Offene Nennungen \\
\hline ST10 & 20 & $\begin{array}{l}\text { Welche Lösungen könnten mithilfe offener } \\
\text { öffentlicher Einkaufsdaten realisiert - und welche } \\
\text { Märkte beziehungsweise Geschäftsmodelle } \\
\text { erschlossen werden? }\end{array}$ & Offene Nennungen \\
\hline ST09 & 21 & $\begin{array}{l}\text { Welche wirtschaftlichen, sozialen oder politischen } \\
\text { Impulse können Sie sich durch eine Öffnung } \\
\text { öffentlicher Einkaufsdaten vorstellen? }\end{array}$ & $\begin{array}{l}\text { Rangordnung mit } 3 \\
\text { Rangplätzen }\end{array}$ \\
\hline OG01 & 22 & $\begin{array}{l}\text { Wer könnte welchen Nutzen aus offenen } \\
\text { öffentlichen Einkaufsdaten ziehen? }\end{array}$ & Skala mit 3 Werten \\
\hline ST05 & 23 & $\begin{array}{l}\text { Welche Stärken sehen Sie in der (weiteren) Öffnung } \\
\text { öffentlicher Einkaufsdaten? }\end{array}$ & Mehrfachauswahl \\
\hline ST06 & 24 & $\begin{array}{l}\text { Welche Schwächen sehen Sie in der (weiteren) } \\
\text { Öffnung öffentlicher Einkaufsdaten? }\end{array}$ & Mehrfachauswahl \\
\hline ST03 & 25 & $\begin{array}{l}\text { Wo sehen Sie die größten Chancen in der (weiteren) } \\
\text { Öffnung öffentlicher Einkaufsdaten? }\end{array}$ & Mehrfachauswahl \\
\hline ST04 & 26 & $\begin{array}{l}\text { Welche Risiken sehen Sie in der (weiteren) Öffnung } \\
\text { öffentlicher Einkaufsdaten? }\end{array}$ & Mehrfachauswahl \\
\hline
\end{tabular}

3. Rand- und Rahmenbedingungen

Tabelle 8.4 Zuordnung der Fragen auf den Abschnitt Rand- und Rahmenbedingungen

\begin{tabular}{l|l|l|l}
\hline Code & Nr. & Frage & Antworttyp \\
\hline TR01 & 27 & $\begin{array}{l}\text { Inwieweit kann die Öffnung der Einkaufsdaten zu } \\
\text { mehr Transparenz führen? }\end{array}$ & Mehrfachauswahl \\
\hline PA01 & 28 & $\begin{array}{l}\text { Durch welche Maßnahmen in der Beteiligung Dritter } \\
\text { kann die Legitimation von öffentlichen Einkaufsent- } \\
\text { scheidungen in der Zukunft gestützt beziehungsweise } \\
\text { verbessert werden? }\end{array}$ & Mehrfachauswahl \\
\hline
\end{tabular}


Tabelle 8.4 (Fortsetzung)

\begin{tabular}{|c|c|c|c|}
\hline Code & Nr. & Frage & Antworttyp \\
\hline TR05 & 29 & $\begin{array}{l}\text { In welchem Teil des Gesamtprozesses der } \\
\text { Beschaffung kann Ihrer Meinung nach durch die } \\
\text { Öffnung von öffentlichen Einkaufsdaten Korruption } \\
\text { am ehesten reduziert werden? }\end{array}$ & $\begin{array}{l}\text { Schieberegler mit } \\
11 \text { Zwischenwerten } \\
(0 \%-100 \%)\end{array}$ \\
\hline TR06 & 30 & $\begin{array}{l}\text { Welche weiteren, flankierenden Maßnahmen sind } \\
\text { über die Öffnung des öffentlichen Einkaufswesens } \\
\text { hinweg aus Ihrer Sicht notwendig, um Transparenz } \\
\text { nachhaltig abzusichern beziehungsweise Korruption } \\
\text { einzudämmen? }\end{array}$ & Mehrfachauswahl \\
\hline KO01 & 31 & $\begin{array}{l}\text { Welche Mechanismen eignen sich gut, um das } \\
\text { Engagement verschiedener Beteiligter rund um } \\
\text { offene öffentliche Einkaufsdaten zu fördern? }\end{array}$ & Mehrfachauswahl \\
\hline OG03 & 32 & $\begin{array}{l}\text { Sind aus Ihrer Sicht zusätzliche Ressourcen (zum } \\
\text { Beispiel Mitarbeiter, Budget) zur Öffnung des } \\
\text { öffentlichen Einkaufswesens über die vorhandenen } \\
\text { Bordmittel hinaus erforderlich? }\end{array}$ & Auswahl \\
\hline RE01 & 33 & $\begin{array}{l}\text { Was hat sich aus Ihrer Sicht mit der Umsetzung der } \\
\text { EU-Vergaberichtlinien (2014/23/EU, 2014/24/EU } \\
\text { und 2014/25/EU) in nationales Recht in 2016/2017 } \\
\text { bezüglich der Offenlegung von öffentlichen } \\
\text { Einkaufsdaten verändert - was wird sich noch ver- } \\
\text { ändern? }\end{array}$ & Offene Texteingabe \\
\hline RE02 & 34 & $\begin{array}{l}\text { Was müssten weitere europäische und nationale } \\
\text { Gesetzgebungen, Richtlinien und Verordnungen } \\
\text { noch beinhalten, um die Vision eines offenen } \\
\text { öffentlichen Einkaufs Realität werden zu lassen? }\end{array}$ & Offene Texteingabe \\
\hline AG09 & 35 & $\begin{array}{l}\text { Ist das Transparenzgesetz in Hamburg als Vorreiter } \\
\text { oder Blaupause aus Ihrer Sicht ein guter Vorreiter } \\
\text { und als vorbildhafte Umsetzung geeignet? Warum } \\
\text { beziehungsweise warum nicht? }\end{array}$ & Auswahl \\
\hline AG04 & 36 & $\begin{array}{l}\text { Welche Projekte beziehungsweise vorbildhaften } \\
\text { Umsetzungen (Best Practices) bezogen auf die } \\
\text { Öffnung öffentlicher Einkaufsdaten kennen Sie oder } \\
\text { sollten wir noch ansprechen? }\end{array}$ & Offene Texteingabe \\
\hline OG05 & 37 & $\begin{array}{l}\text { Welchen Zeithorizont halten Sie für die Öffnung } \\
\text { des öffentlichen Einkaufswesens in Deutschland für } \\
\text { realistisch? }\end{array}$ & Auswahl \\
\hline
\end{tabular}


4. Feedback

Tabelle 8.5 Zuordnung der Fragen auf den Abschnitt Feedback

\begin{tabular}{l|l|l|l}
\hline Code & Nr. & Frage & Antworttyp \\
\hline AG06 & 38 & $\begin{array}{l}\text { Können Sie uns weitere Experten rund um die } \\
\text { Öffnung öffentlicher Einkaufsdaten nennen? } \\
\text { Wen sollten wir in diesem Kontext noch } \\
\text { befragen? } \\
\text { [Antworten nicht in der Auswertung dargestellt!] }\end{array}$ & Offene Texteingabe \\
\hline AG05 & 39 & $\begin{array}{l}\text { Haben Sie sonstige Kommentare, Feedback oder } \\
\text { Anregungen für uns? } \\
\text { [Antworten nicht in der Auswertung dargestellt! }\end{array}$ & Offene Texteingabe \\
\hline AG10 & 40 & $\begin{array}{l}\text { Und zu guter Letzt: Haben Sie Interesse am } \\
\text { Ergebnis dieser Studie? } \\
\text { [Antworten nicht in der Auswertung dargestellt!] }\end{array}$ & $\begin{array}{l}\text { Getrennte Erhebung von } \\
\text { Kontaktdaten }\end{array}$ \\
\hline
\end{tabular}

Im Fokus der Umfrage stand die Untersuchung der Bereitstellung von Einkaufsdaten vor und nach der Vergabeentscheidung (ex-ante und ex-post) für Deutschland. Relevant waren nationale Ausschreibungen und Vergaben, also der Unterschwellenbereich. Dabei wurde der gesamte Prozess des öffentlichen Einkaufs betrachtet (über die Teilprozesse Planung, Ausschreibung, Bewertung, Vergabe und Ausführung). Im Vordergrund stand die Nachfragesicht, das heißt das aktuelle und künftige Handeln auf Verwaltungsebene mit seinem Einfluss auf die verschiedenen Beteiligten. In den Fragen mit vorgegebenen Antwortmöglichkeiten wurde nicht nach Bund, Ländern und Kommunen unterschieden.

\subsubsection{Auswahl der Stichprobe}

Hinsichtlich der Populationsgröße wurde die Annahme getroffen, dass man im Umfeld der öffentlichen Beschaffung bei circa 11000 Kommunen (Rudnicka, 2019) von mindestens 1000 Personen ausgehen kann, die mit diesem Thema befasst sind. Es handelt sich um eine offene Grundgesamtheit, da die Zahl nicht abschließend und eindeutig bestimmt werden kann (Kuckartz et al., 2012, S. 52). Als Konfidenzniveau wurde $90 \%$ gewählt, als Fehlerspanne $15 \%$. Das Konfidenzniveau gibt an, inwieweit die Antworten der Stichprobe die Ansichten der Population präzise reflektieren. Die Fehlerspanne benennt das Ausmaß, in welchem die Antworten der Stichprobe von jenen der Population abweichen dürfen. Das Konfidenzniveau wurde etwas niedriger angesetzt und die Fehlerspanne etwas 
höher als üblich, da es sich bei dem untersuchten Thema um ein Nischenthema handelt, welches noch stark diskutiert wird, auch in Abhängigkeit der Betroffenheit einzelner Gruppen. Das heißt, hier sind mit hoher Wahrscheinlichkeit Ausreißer in den Ansichten möglich. Mit diesen Angaben ergibt sich eine Stichprobengröße von 30 Personen. Diese ändert sich auch nicht bei Vergrößerung der Population über die 1000 Personen hinaus, das bedeutet, selbst mit Bezug auf eine Populationsgröße von 1 Million Personen bleibt die benötigte Stichprobengröße konstant bei 30 benötigten Stichproben. Angeschrieben wurden 161 Führungskräfte und Experten mit einer Verteilung gemäß Tabelle 8.6 (eigene Gruppenzuordnung).

Tabelle 8.6 Anzahl Eingeladene nach Gruppen

\begin{tabular}{l|c|c}
\hline Gruppe & Einladung (Anzahl) & Einladung (\%) \\
\hline Verwaltung & 75 & $47 \%$ \\
\hline Wirtschaft & 38 & $24 \%$ \\
\hline NGO & 29 & $18 \%$ \\
\hline Wissenschaft & 12 & $7 \%$ \\
\hline Journalismus/Medien & 7 & $4 \%$ \\
\hline Sonstige & 0 & $0 \%$ \\
\hline Summe & $\mathbf{1 6 1}$ & $\mathbf{1 0 0} \%$ \\
\hline
\end{tabular}

Voraussetzung für eine gute Analyse ist es, die Stichprobe in strukturellen und für die Grundgesamtheit relevanten Merkmalen möglichst real abzubilden (Friedrichs, 1990, S. 243 ff.).

Bei den Einladungen sind Wissenschaft und Journalismus/Medien anteilig schwächer vertreten - diese Verteilung entspricht einem zufriedenstellenden Abbild der Grundgesamtheit. Es liegt nahe, dass in dieser Grundgesamtheit Verwaltungen, Wirtschaft und NGOs den größeren Anteil stellen: Verwaltungen versuchen regelmäßig, ihre Bedarfe über Ausschreibungen am Markt zu decken, NGOs als Vertreter der Zivilgesellschaft treten in diesem Umfeld wiederholt für Transparenz ein.

\subsubsection{Bereitstellung des Fragebogens}

Die Auswahl der Teilnehmer erfolgte mit Blick auf folgenden Erfahrungshintergrund (Kombinationen möglich):

- Öffentlicher Einkauf/öffentliche Beschaffung

- Offene Regierungsdaten (Open Government Data) 
- Digitalisierung/E-Government/Big Data im öffentlichen Sektor

- Verwaltung/Regierung; Wirtschaft; Medien/Journalismus; Wissenschaft; NGO

Es handelt sich folglich um ein nicht-zufallsgesteuertes Verfahren mit einer bewusst gewählten Stichprobe, da die Auswahlkriterien den Teilnehmerkreis einschränkten und nicht dem Zufall überließen (Kuckartz et al., 2012, S. 52).

Tabelle 8.7 sind die eingeladenen Teilnehmer nach Organisationszuordnung zu entnehmen. Im Verwaltungsbereich wurden sowohl Vertreter von Bund, Ländern und Kommunen angefragt; im Wirtschaftsbereich Verbände sowie Industrie- und Handelskammern, Beratungsgesellschaften, Anwaltskanzleien sowie Anbieter von Vergabelösungen. Bei den NGOs erstrecken sich die Eingeladenen von Webdesignern über IT-Entwickler, Journalisten, Hackervereinigungen, Projekte, Vertreter offenen und transparenten Regierungshandelns bis hin zu Organisationen, die eine größere Informations- und Meinungsfreiheit einfordern. Die Wissenschaft ist durch Forschungsinstitutionen vertreten, die sich ebenfalls mit den oben genannten Themenkomplexen bereits tiefer befasst haben. Gleiches gilt für die Vertreter der Medien.

Tabelle 8.7 Organisationszuordnung der Teilnehmer

\begin{tabular}{l|l|l}
\hline Wissenschaft & Verwaltung & Wirtschaft \\
\hline - Fachhochschule für & - Bundesamt für Ausrüstung, & - Aulinger Rechtsanwälte \\
öffentliche Verwaltung, & Informationstechnik und & - Blomstein Rechtsanwälte \\
Köln und Bielefeld & Nutzung der Bundeswehr & - Bundesverband Digitale \\
- Fraunhofer Fokus, Berlin & - BMI & Wirtschaft \\
- Freie Universität, Berlin & - Bundesministerium für & - Capgemini GmbH \\
- Technische Informations- & Wirtschaft und Technologie & - Cosinex GmbH \\
bibliothek, Hannover & - Deutsches Vergabeportal & - Deloitte GmbH \\
- Universität für Ver- & (DTVP) GmbH & - Healey Hudson GmbH \\
waltungswissenschaften, & - Deutsches Vergabenetz- & - DIHK e. V. \\
Speyer & werk (DVNW) GmbH & - Ernst \& Young AG \\
- Universität Koblenz- & - KOINNO & - Diverse IHKen \\
Landau & - IT-Planungsrat & - Institut für den \\
- Johannes Kepler Uni- & - Länder Hamburg, Bremen, & öffentlichen Sektor e. V. \\
versität, Linz & NRW, Hessen, & - Kienbaum Consultants \\
- Universität Twente & - Deutscher Landkreistag & International GmbH \\
- Zeppelin Universität, Fried- & - DStGB e. V. & - KPMG AG \\
richshafen & - Kommunale Gemein- & - PwC GmbH \\
& schaftsstelle & - subreport Verlag Schawe \\
& - Kommunen Bonn, Köln, & GmbH \\
& Moers & - Zentralverband des \\
& - Diverse Vergabestellen & deutschen Handwerks \\
& - Vitako e. V. & e. V. \\
\hline
\end{tabular}


Tabelle 8.7 (Fortsetzung)

\begin{tabular}{l|l|l}
\hline NGO & Medien/Journalismus & \\
\hline - Chaos Computer Club e. V. & - Netzpolitik.org e. V. & - \\
- Digitale Gesellschaft e. V. & - Zeitschrift ,eGovernment & \\
- Digiwhist (Projekt) & $\begin{array}{l}\text { Computing“ } \\
\text { - Forum Informationsfreiheit } \\
\text { (FOI) }\end{array}$ & $\begin{array}{c}\text { Zeitschrift „der Gemeinde- } \\
\text { rat“ }\end{array}$ \\
- Kommune 2.0 e. V. & & \\
- Mehr Demokratie e. V. & \\
- Offene Kommunen.NRW & \\
Institut e. V. & \\
- OKFN e. V. & \\
- OCCRP (Projekt) & \\
- TI Deutschland e. V. & \\
- Verein Deutscher & \\
Ingenieure e. V. & & \\
\hline
\end{tabular}

Die Teilnehmer wurden per E-Mail zur Befragung eingeladen. Im Einladungstext befand sich ein Link zur Befragung, der mit einem individuellen Zugangsschlüssel versehen war. Mehrfachteilnahmen konnten so ausgeschlossen werden. Des Weiteren konnte die Befragung unterbrochen, zwischengespeichert und zu einem späteren Zeitpunkt fortgesetzt werden. Der Fragebogen war vom 18. Oktober bis 22. Dezember 2018 online zugänglich:

- Welle 1: 18. Oktober bis 02. November 2018

- Welle 2: 02. November bis 28. November 2018

- Welle 3: 29. November bis 22. Dezember 2018

Die Datenerhebung erfolgte anonymisiert; ein Rückschluss von den eingegebenen Daten auf den Teilnehmer oder umgekehrt ist nicht möglich. Es war den Teilnehmenden möglich, eine Mailadresse zu hinterlassen, um auf Wunsch die Ergebnisse der Befragung zu erhalten.

\subsubsection{Rücklaufquote, Auswertung und eingesetzte Software}

Von 161 verschickten Fragebögen wurden 36 vollständig beantwortet. Damit liegt die Rücklaufquote bei 22,5 \%. Die Stichprobe repräsentiert damit die Population beim gewählten Konfidenzniveau und bei der gewählten Fehlerspanne.

Mit Blick auf die Teilnahme gemäß Tabelle 8.8 sind NGOs und Wissenschaft im Vergleich zur Einladung prozentual leicht überrepräsentiert; Ver- 
Tabelle 8.8 Eingeladene und Teilnehmer der Studie im Vergleich

\begin{tabular}{l|c|c|c|c|c}
\hline Gruppe & $\begin{array}{c}\text { Einladung } \\
\text { (Anzahl) }\end{array}$ & $\begin{array}{c}\text { Einladung/ } \\
\mathbf{1 6 1}\end{array}$ & $\begin{array}{c}\text { Teilnahme } \\
\text { (Anzahl) }\end{array}$ & $\begin{array}{c}\text { Teilnahme/ } \\
\mathbf{3 6}\end{array}$ & $\begin{array}{c}\text { Teiln. Anz./ } \\
\text { Einl. Anz. }\end{array}$ \\
\hline Verwaltung & 75 & $47 \%$ & 14 & $39 \%$ & $19 \%$ \\
\hline Wirtschaft & 38 & $24 \%$ & 7 & $20 \%$ & $18 \%$ \\
\hline NGO & 29 & $18 \%$ & 9 & $25 \%$ & $31 \%$ \\
\hline Wissenschaft & 12 & $7 \%$ & 3 & $8 \%$ & $25 \%$ \\
\hline Medien & 7 & $4 \%$ & 1 & $3 \%$ & $14 \%$ \\
\hline Sonstige & 0 & $0 \%$ & 2 & $6 \%$ & n./r. \\
\hline Summe & $\mathbf{1 6 1}$ & $\mathbf{1 0 0} \%$ & $\mathbf{3 6}$ & $\mathbf{1 0 0 \%}$ & $\mathbf{1 0 0} \%$ \\
\hline
\end{tabular}

waltungen, Wirtschaft und Journalismus/Medien leicht unterrepräsentiert. Dies lässt einen vorsichtigen Rückschluss auf das etwas höhere beziehungsweise niedrigere Interesse am Thema zu. Insgesamt kann mit 22,5\% Rücklaufquote und in Anbetracht des langen Fragebogens mit insgesamt 40 Fragen eine überdurchschnittliche Beteiligung ${ }^{1}$ und damit ein solides Interesse am Thema festgestellt werden. Die Ergebnisse lassen einen substanziellen Beitrag zur Formulierung des Leitbilds der Öffnung öffentlicher Einkaufsdaten erwarten.

In der anschließenden Auswertung wurden nur vollständig ausgefüllte Fragebögen berücksichtigt. Bei den Auswertungen handelt es sich in der Regel um Häufigkeitsverteilungen oder Berechnungen von Mittelwerten. Bei den Häufigkeitsverteilungen wurde die gesamte Stichprobe mit $n=36$ Fällen zugrunde gelegt und dann relative Prozentauswertungen berechnet. Auf Dezimalstellen wurde in den Diagrammen zum Zweck besserer Lesbarkeit verzichtet.

Bei den Fragetypen, die mittels Schieberegler auf einer Skala von $0 \%$ bis $100 \%$ über elf Stufen zu beantworten waren, wurden die Ergebnisse wie folgt zusammengefasst:

- Negativ: $0 \%, 10 \%, 20 \%, 30 \%$

- Mittel: $40 \%, 50 \%, 60 \%$

- Positiv: $70 \%, 80 \%, 90 \%, 100 \%$

${ }^{1}$ Zum Vergleich und zur Einordnung der Rücklaufquote mit Studien rund um den öffentlichen Einkauf: Erfassung des aktuellen Standes der innovativen öffentlichen Beschaffung in Deutschland 2018: 3220 Befragte, Rücklauf von 182 (=5,6\%) (Schaupp \& Eßig, 2018, S. 4); Kommunale Beschaffung im Umbruch: 174 Befragte, Rücklauf von 56 (=32,5\%) (Beck \& Schuster, 2013, S. 20); "Einkäufer Staat" als Innovationstreiber: 2306 Befragte, Rücklauf von 265 (=11,5\%) (Lorenz et al., 2009, S. 77); Digitale Beschaffung in der öffentlichen Verwaltung: Etwa 500 Befragte, Rücklauf von 155 (=31\%) (Thome et al., 2007, S. 5); Public e-Procurement: 600 Befragte, Rücklauf von 44 (=7,33 \%) (Jansen, 2001, S. 8). 
Die Antworten auf offene Fragen wurden gesondert ausgewertet. Es erfolgte wenn möglich - die Clusterung in Themengebiete und ebenfalls die Nutzung von Häufigkeitsverteilungen.

Einige Fragen haben in ihrer Darstellung sowohl eine Tabelle nach Antworthäufigkeit wie auch nach Antwortreihenfolge im Fragebogen. Dies dient lediglich der besseren Lesbarkeit.

Antworten auf die Fragen zum letzten Teil des Fragebogens (Feedback) mit den Fragen 38 (AG06; Best Practices), 39 (AG05; Feedback zum Fragebogen) und 40 (AG10; freiwillige Angabe der E-Mail) wurden hier nicht dargestellt, da sie für die inhaltliche Auswertung der Studienergebnisse nicht unmittelbar relevant sind. Diese Antworten wurden jedoch mit Blick auf den Fortschritt der Dissertation im Rahmen persönlicher Gespräche erörtert beziehungsweise mit Blick auf die Lehre berïcksichtigt.

In Bezug auf die eingesetzte Software erfordert die Nutzung einer Online-Erhebung den Einsatz unterschiedlicher IT-Anwendungen. Dies betrifft die Erstellung des Fragebogens, die statistische Aufbereitung der Daten sowie abschließend die leserfreundliche Visualisierung der Ergebnisse. Für den vorliegenden Online-Fragebogen wurde Sosci-Survey (Version 3.1.06-i) genutzt. Die Programmierung einzelner Fragen erfolgte in PHP und HTML. Die statistische Aufbereitung der Daten erfolgte in Maxqda Analytics Pro und Maxqda Stats (Version 18). Mithilfe von Microsoft Excel (für Microsoft Office 365 ProPlus) erfolgte die Weiterverarbeitung der Daten in Hilfstabellen und die anschließende grafische Aufbereitung.

\subsubsection{Abschließender Workshop zur Diskussion der Studienergebnisse}

Am 05. Juli 2019 wurde der abschließende Experten-Workshop mit insgesamt acht Teilnehmern durchgeführt. Die Teilnehmer repräsentierten hier die Wirtschaft, Verwaltung, NGOs und Wissenschaft. Die Agenda beinhaltete die Darstellung der Studienergebnisse und anschließend die Diskussion eines möglichen Leitbilds entlang der Kategorien Strategie und Organisation, Recht, Technologie, Transparenz, Partizipation und Kollaboration. Die Anregungen der Teilnehmer wurden anschließend gruppiert und sind in Tabelle 8.9 dargestellt. Eine wesentliche Anregung war, Mehrwerte für die jeweiligen Zielgruppen herauszustellen sowie Best Practices auszubauen und besser zu kommunizieren. Ebenfalls sollte Transparenz nicht als alleiniges Ziel, sondern als Mittel zum Zweck verstanden werden. Die Öffnung öffentlicher Einkaufsdaten sollte in den verschiedenen Rechtsgebieten verankert werden und die Partizipation könne möglicherweise über Tools und Auswertungen gesteigert werden. Die Ergebnisse werden in das Leitbild in Kapitel 10 integriert. 
Tabelle 8.9 Rückmeldungen zum Leitbild aus dem Workshop vom 05. Juli 2019

\begin{tabular}{|c|c|}
\hline Kategorie & Inhalte \\
\hline \multirow[t]{4}{*}{$\begin{array}{l}\text { Strategie und } \\
\text { Organisation }\end{array}$} & $\begin{array}{l}\text { Mehrwerte: } \\
\text { - Mehrwerte für die jeweiligen Zielgruppen herausarbeiten } \\
\text { - Geschäftsmodelle definieren } \\
\text { - Vorteile für kleinere Vergabestellen aufzeigen, zum Beispiel Effizienz } \\
\text { steigern und Einsparungen aufzeigen }\end{array}$ \\
\hline & $\begin{array}{l}\text { Integration: } \\
\text { - Diverse Stakeholder in ein gemeinsames Projekt integrieren } \\
\text { - Visualisierungsmöglichkeiten mit den Stakeholdern erarbeiten } \\
\text { - Auswertungen erstellen: Was kostet die Datenerhebung? } \\
\text { - Akteure als Treiber des Wandels verstehen, nicht die Technologie oder } \\
\text { Gesetze }\end{array}$ \\
\hline & $\begin{array}{l}\text { Politische Teilnahme: } \\
\text { - Teilnahme Deutschlands in der OCP und Aufnahme der Forderung der } \\
\text { Öffnung öffentlicher Einkaufsdaten im NAP vorantreiben }\end{array}$ \\
\hline & $\begin{array}{l}\text { Best Practices: } \\
\text { - Sektorenübergreifende Best Practices entwickeln } \\
\text { - „Offenheit braucht Stars, nicht nur ein Regelwerk“ } \\
\text { - Beispiele für Vergabestellen erarbeiten } \\
\text { - OCDS und Digiwhist nutzen }\end{array}$ \\
\hline Recht & $\begin{array}{l}\text { - Öffnung öffentlicher Einkaufsdaten im Recht verankern } \\
\text { - Datenschutzrecht mit Blick auf Geschäftsgeheimnisse reformieren } \\
\text { - Neue PSI-Richtlinie interpretieren und umsetzen, zum Beispiel im } \\
\text { IWG/IFG }\end{array}$ \\
\hline \multirow[t]{4}{*}{ Technologie } & $\begin{array}{l}\text { Umfassende Digitalisierung: } \\
\text { - Alle Teilprozesse mit ganzheitlichem Ansatz digitalisieren }\end{array}$ \\
\hline & $\begin{array}{l}\text { Umfang der Datenöffnung: } \\
\text { - Umfang klar umreißen: Unterschwelle, welche Daten genau, welche } \\
\text { Datenqualität wird gewünscht } \\
\text { - Öffentliche Einkaufsdaten mit anderen Datensätzen verknüpfen }\end{array}$ \\
\hline & $\begin{array}{l}\text { Technische Standards: } \\
\text { - Technische Standards für Open Data nutzen } \\
\text { - REST API statt Datei verwenden; strukturierte und einheitliche } \\
\text { Formate forcieren }\end{array}$ \\
\hline & $\begin{array}{l}\text { Vorgehen: } \\
\text { - Schrittweises Vorgehen über die Länder etablieren }\end{array}$ \\
\hline
\end{tabular}


Tabelle 8.9 (Fortsetzung)

\begin{tabular}{|c|c|}
\hline Kategorie & Inhalte \\
\hline \multirow[t]{2}{*}{ Transparenz } & $\begin{array}{l}\text { Transparenz als Mittel, nicht als alleiniges Ziel: } \\
\text { - Transparenz ist oft angstbesetzt - Aufklärung, dass es um } \\
\text { Optimierung und nicht Anprangern geht } \\
\text { - Anderes Framing nutzen - eher Effizienz und Information? } \\
\text { - Mehrwerte für die jeweilige Zielgruppe aufzeigen } \\
\text { - Transparenz als Chance für die Verwaltung begreifen und hervor- } \\
\text { heben }\end{array}$ \\
\hline & $\begin{array}{l}\text { Konkrete Umsetzung: } \\
\text { - Ansätze wie Digiwhist nutzen } \\
\text { - Gute Beispiele aufbauen statt „,naming and shaming“ } \\
\text { - Transparenz als natürlichen Teil des Vergabeprozesses etablieren } \\
\text { - Geheimhaltung benötigt Erklärung und nicht anders herum } \\
\text { - Tools und Microservices für bessere Auswertungen bereitstellen }\end{array}$ \\
\hline \multirow[t]{3}{*}{$\begin{array}{l}\text { Partizipation und } \\
\text { Kollaboration }\end{array}$} & $\begin{array}{l}\text { Zentraler Zugriff: } \\
\text { - Alle Daten auf einem Portal veröffentlichen } \\
\text { - Zentralen Zugriff schaffen und Mehrwerte für Startups hervorheben }\end{array}$ \\
\hline & $\begin{array}{l}\text { Tools und Auswertungen: } \\
\text { - Benchmarking - Möglichkeiten des Vergleichs nutzen } \\
\text { - Tools schaffen, von denen auch die öffentliche Hand selbst profitieren } \\
\text { kann } \\
\text { - Daten für die Öffentlichkeit aufbereiten (was kann man mit den Daten } \\
\text { machen) } \\
\text { - NGOs und Medien als Intermediäre in der Auswertung von Daten } \\
\text { nutzen }\end{array}$ \\
\hline & $\begin{array}{l}\text { Best Practices: } \\
\text { - Best Practices für Informationsaustausch zwischen öffentlichen Auf- } \\
\text { traggebern schaffen }\end{array}$ \\
\hline
\end{tabular}

\subsection{Studienergebnisse}

Die Studienergebnisse werden in diesem Kapitel vorgestellt und erörtert. Fragen, bei denen eine Mehrfachauswahl oder eine offene Texteingabe möglich war, sind als solche gekennzeichnet. Für die weiteren Zuordnungen sei auf Abschnitt 8.1 beziehungsweise Abschnitt 8.1.2 verwiesen. 


\subsubsection{Ausgangssituation}

Frage 1: Wie umfangreich ist Ihre Erfahrung im öffentlichen Einkauf?

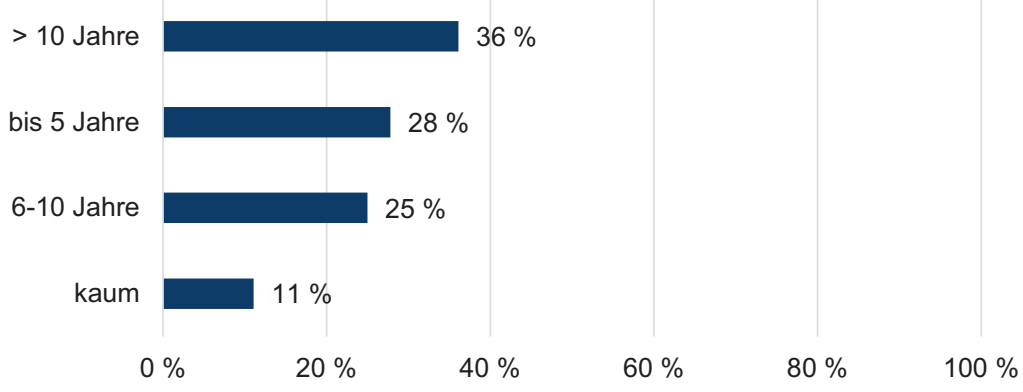

Abbildung 8.1 Erfahrung der Teilnehmer im öffentlichen Einkauf

Tabelle 8.10 Erfahrung nach Antworthäufigkeit

\begin{tabular}{l|c|c}
\hline Antworten & Prozent & Anzahl \\
\hline$>$ 10 Jahre & $36 \%$ & 13 \\
\hline bis 5 Jahre & $28 \%$ & 10 \\
\hline 6-10 Jahre & $25 \%$ & 9 \\
\hline kaum & $11 \%$ & 4 \\
\hline Summe &. & $\mathbf{3 6}$ \\
\hline
\end{tabular}

Tabelle 8.11 Erfahrung nach Antwortreihenfolge

\begin{tabular}{l|c|c}
\hline Antworten & Prozent & Anzahl \\
\hline kaum & $11 \%$ & 4 \\
\hline bis 5 Jahre & $28 \%$ & 10 \\
\hline 6-10 Jahre & $25 \%$ & 9 \\
\hline > 10 Jahre & $36 \%$ & 13 \\
\hline Summe &.$\%$ & $\mathbf{3 6}$ \\
\hline
\end{tabular}




\section{Bewertung:}

Auf die Frage, über welchen Erfahrungshorizont die Teilnehmer im öffentlichen Einkauf verfügen, antworten $61 \%$, dass sie mehr als sechs Jahre Erfahrung vorweisen können, $28 \%$ weisen bis zu fünf Jahre Erfahrung auf. Nur $11 \%$ der Teilnehmer besitzen kaum Erfahrung im öffentlichen Einkauf. Das bedeutet, dass der überwiegende Teilnehmerkreis dieser Studie aus (sehr) fachkundigen Führungskräften und Experten besteht (Abbildung 8.1/Tabelle 8.10/Tabelle 8.11).

\section{Frage 2: Zu welcher Gruppe zählen Sie sich?}

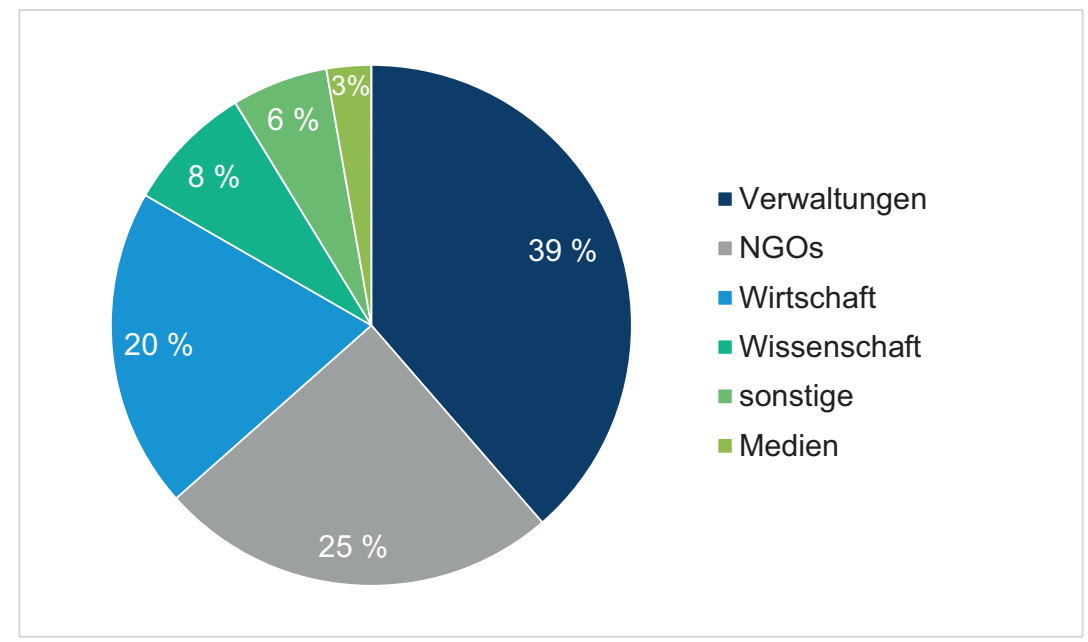

Abbildung 8.2 Gruppenzugehörigkeit der Teilnehmer

Tabelle 8.12 Gruppenzugehörigkeit der Teilnehmer

\begin{tabular}{l|c|c}
\hline Antworten & Prozent & Anzahl \\
\hline Verwaltungen & $39 \%$ & 14 \\
\hline NGOs & $25 \%$ & 9 \\
\hline Wirtschaft & $14 \%$ & 5 \\
\hline sonstige & $11 \%$ & 4 \\
\hline Wissenschaft & $8 \%$ & 3 \\
\hline Medien & $3 \%$ & 1 \\
\hline Summe &.$\%$ & $\mathbf{3 6}$ \\
\hline
\end{tabular}




\section{Bewertung:}

Die befragten Gruppen sind in der Studie unterschiedlich repräsentiert. Den größten Anteil mit $39 \%$ stellen die Verwaltungen, gefolgt von den NGOs mit $25 \%$ sowie der Wirtschaft mit $14 \%$ dar. Im kleineren Maße sind hingegen die Teilnehmer aus Wissenschaft $(11 \%)$ und Medien $(3 \%)$ repräsentiert (siehe Abbildung 8.2/Tabelle 8.12). Dies spiegelt annähernd die Verteilung der Einladungen wider (siehe Abschnitt 8.1.3).

Unter „sonstige“ wurden noch Dienstleister/eVergabe-Anbieter, Privatwirtschaft (eVergabelösungs-Anbieter), Verwaltung und Verband sowie Rechtsberatung genannt.

Frage 3: Inwieweit beschäftigen Sie sich bereits mit „Offenen Verwaltungsdaten" beziehungsweise "Open Government Data"?

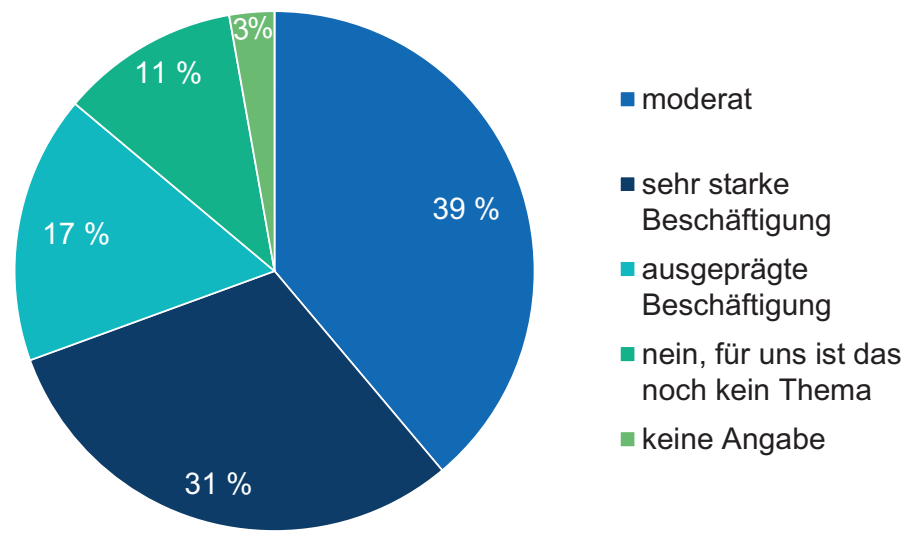

Abbildung 8.3 Beschäftigung mit der Veröffentlichung öffentlicher Verwaltungsdaten 
Tabelle 8.13 Beschäftigung mit dem Thema nach Antworthäufigkeit

\begin{tabular}{l|c|c}
\hline Antworten & Prozent & Anzahl \\
\hline moderat & $39 \%$ & 14 \\
\hline sehr starke Beschäftigung & $31 \%$ & 11 \\
\hline ausgeprägte Beschäftigung & $17 \%$ & 6 \\
\hline $\begin{array}{l}\text { nein, für uns ist das noch kein } \\
\text { Thema }\end{array}$ & $11 \%$ & 4 \\
\hline keine Angabe & $3 \%$ & 1 \\
\hline Summe &. & $\mathbf{3 6}$ \\
\hline
\end{tabular}

Tabelle 8.14 Beschäftigung mit dem Thema nach Antwortreihenfolge

\begin{tabular}{l|c|c}
\hline Antworten & Prozent & Anzahl \\
\hline sehr starke Beschäftigung & $31 \%$ & 11 \\
\hline ausgeprägte Beschäftigung & $17 \%$ & 6 \\
\hline moderat & $39 \%$ & 14 \\
\hline $\begin{array}{l}\text { nein, für uns ist das noch kein } \\
\text { Thema }\end{array}$ & $11 \%$ & 4 \\
\hline keine Angabe & $3 \%$ & 1 \\
\hline Summe &. & $\mathbf{3 6}$ \\
\hline
\end{tabular}

\section{Bewertung:}

Auf die Frage, inwieweit sich die Teilnehmer bereits mit „Offenen Verwaltungsdaten" befassen, antworten $87 \%$ der Teilnehmer, dass sie sich ausgeprägt, sehr stark und moderat mit offenen Verwaltungsdaten befassen; nur $11 \%$ der Befragten beschäftigen sich noch nicht mit diesem Thema (Abbildung 8.3/ Tabelle 8.13/Tabelle 8.14). 
Frage 4: Gibt es bei Ihnen in Bezug auf die Öffnung der öffentlichen Einkaufsdaten bereits Ansätze, Initiativen oder Programme, um dies zu fördern/ zu nutzen?

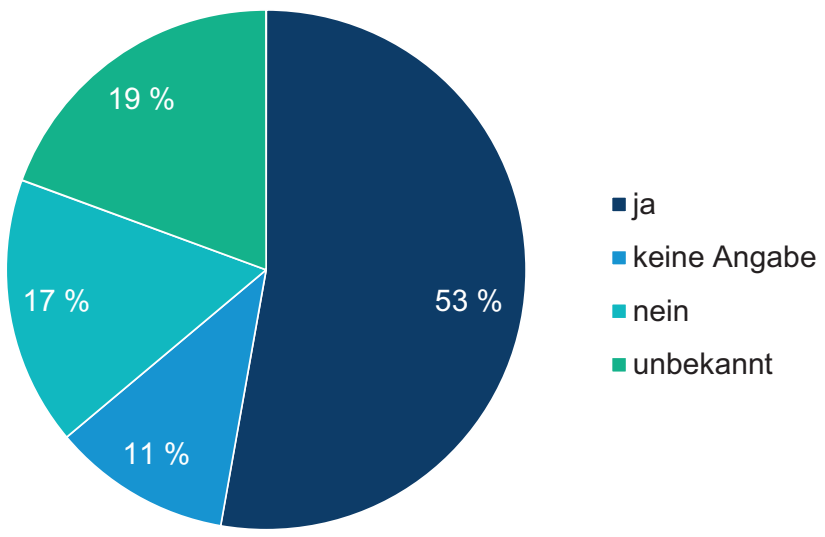

Abbildung 8.4 Förderung der Öffnung der öffentlichen Einkaufsdaten

Tabelle 8.15 Förderung der Öffnung der öffentlichen Einkaufsdaten

\begin{tabular}{l|c|c}
\hline Antworten & Prozent & Anzahl \\
\hline ja & $53 \%$ & 19 \\
\hline unbekannt & $19 \%$ & 7 \\
\hline nein & $17 \%$ & 6 \\
\hline keine Angabe & $11 \%$ & 4 \\
\hline Summe &. & $\mathbf{3 6}$ \\
\hline
\end{tabular}

\section{Bewertung:}

Auf die Frage, ob es bei den Teilnehmern in Bezug auf die Öffnung der öffentlichen Einkaufsdaten bereits Ansätze, Initiativen oder Programme gibt, um diese zu fördern/zu nutzen, antwortet die Mehrheit von $53 \%$ der Befragten, dass sie bereits Ansätze gestartet hat (siehe Abbildung 8.4/Tabelle 8.15). Die Nennungen wurden Clustern zugeordnet. Diese reichen von ersten Überlegungen 
über das Engagement in Initiativen bis zu konkreten Bekanntmachungen öffentlicher Einkaufsdaten auf diversen Portalen. Unter „Überlegungen“ fallen zum Beispiel Ansätze, öffentlichen Auftraggebern mithilfe der öffentlichen Einkaufsdaten eine Preisindikation abhängig von Jahreszeit und Region zu geben. Um Behördenentscheidungen nachvollziehbarer $\mathrm{zu}$ machen, wird an anderer Stelle überlegt, sich aktiv für Informationsfreiheits- und Transparenzgesetze einzusetzen. Unter der „Mitwirkung in Initiativen“ wird unter anderem die Mitarbeit in der Initiative „Digiwhist“ angegeben, aber auch auf EU-Initiativen wie beispielsweise den EU Datathon (siehe https://publications.europa.eu/en/ web/eudatathon) verwiesen. Eine Nennung umfasste auch den Hinweis auf das Humanist Institute for Cooperation with Developing Countries (HIVOS), welches seit 2016 ein internationales Programm zu Open Contracting anbietet (siehe https://www.openupcontracting.org). Weiters wird auf die Projekte mit dem Land NRW verwiesen (hier Vergabe.NRW und Open.NRW). Unter „Bekanntmachungen auf Portalen“ sind die meisten Nennungen zu verzeichnen. Zugunsten der Übersichtlichkeit sind sie in Tabelle 8.16 zusammengefasst:

Tabelle 8.16 Varianten der Bekanntmachungen auf Portalen

\begin{tabular}{|c|c|}
\hline Nr. & Varianten der Bekanntmachungen auf Portalen \\
\hline 1 & $\begin{array}{l}\text { Beabsichtigte Vergaben/Ausschreibungen und vergebene Aufträge im Rahmen von } \\
\text { freihändigen Vergaben und beschränkten Ausschreibungen }\end{array}$ \\
\hline 2 & $\begin{array}{l}\text { Jährlicher Vergabebericht mit Angabe zum Umfang (Anzahl, Art und Wert der } \\
\text { durchgeführten Vergaben) seit } 1994 \text { (ab } 2004 \text { nur noch elektronisch) }\end{array}$ \\
\hline 3 & $\begin{array}{l}\text { Ausgewählte öffentliche Einkaufsdaten/Informationen als Bestandteil des jähr- } \\
\text { lichen Wirtschaftsberichtes }\end{array}$ \\
\hline 4 & $\begin{array}{l}\text { Zurverfügungstellung der entsprechenden Schnittstellen für offene Daten bei den } \\
\text { eVergabeportalen der Cosinex GmbH, siehe Vergabe.NRW }\end{array}$ \\
\hline 5 & Bereitstellung des internationalen Open Contracting Data Standards \\
\hline 6 & $\begin{array}{l}\text { Für Beschaffungen werden derzeit schon die Bekanntgaben als Open Data veröffent- } \\
\text { licht: https://opendata.bonn.de/dataset/ausschreibungen-vergabeverfahren. Geplant ist } \\
\text { im Jahr } 2019 \text { die Bereitstellung weiterer Vergabeinformationen als Open Data, sodass } \\
\text { das Open Tender-Portal vollumfänglich automatisiert bedient werden kann }\end{array}$ \\
\hline
\end{tabular}

Die Nennungen legen nahe, dass sich die Teilnehmer bislang eher weniger in Projekten organisieren, dafür aber bereits konkreter mit der Bereitstellung der Daten auf Portalen befassen.

Der hohe Anteil (47\%) an den Antworten ,nein“, „,keine Angabe“ und „unbekannt" bedeutet allerdings, dass sich fast die Hälfte der Befragten noch gar nicht mit diesem Thema auseinandersetzt und hier noch Entwicklungspotential besteht. 
Frage 5: Können Sie sich vorstellen, dass die Öffnung öffentlicher Einkaufsdaten als Teil ,Offener Verwaltungsdaten“" Vorteile für Ihre Organisation bieten kann?

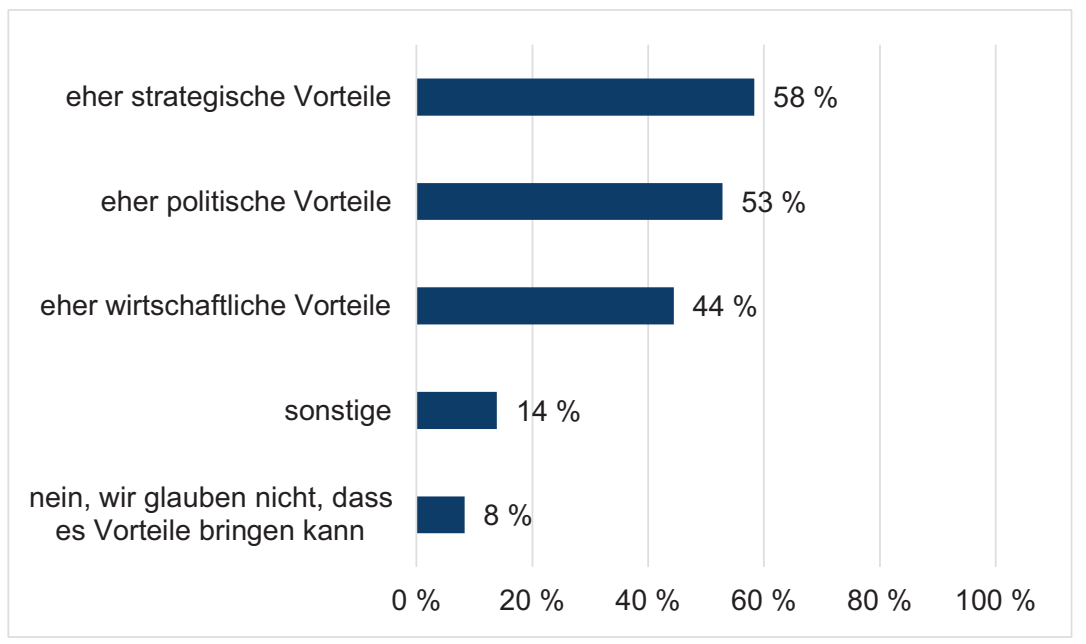

Abbildung 8.5 Wesentliche Vorteile der Öffnung öffentlicher Einkaufsdaten

Tabelle 8.17 Wesentliche Vorteile der Öffnung öffentlicher Einkaufsdaten

\begin{tabular}{l|c|c}
\hline Antworten & Prozent & Anzahl \\
\hline eher strategische Vorteile & $58 \%$ & 21 \\
\hline eher politische Vorteile & $53 \%$ & 19 \\
\hline eher wirtschaftliche Vorteile & $44 \%$ & 16 \\
\hline sonstige & $14 \%$ & 5 \\
\hline nein, wir glauben nicht, dass es & $8 \%$ & 3 \\
Vorteile bringen kann &.$\%$ & $\mathbf{6 4}$ \\
\hline Summe & $\mathbf{3 6 \%}$ & $\mathbf{1 3}$ \\
\hline Mittelwert &
\end{tabular}

\section{Bewertung:}

Auf die Frage (Mehrfachauswahl), ob sich die Teilnehmer vorstellen können, dass die Öffnung öffentlicher Einkaufsdaten als Teil „Offener Verwaltungsdaten" Vorteile für ihre Organisation bieten kann, sieht gemäß Abbildung 8.5/ Tabelle 8.17 nur eine Minderheit von $8 \%$ der Befragten keinen positiven Effekt. 
Die restlichen Antwortmöglichkeiten (strategische, politische, wirtschaftliche Vorteile) werden mit $58 \%, 53 \%$ und $44 \%$ sehr ähnlich bewertet.

In den Nennungen unter ,sonstige“ finden sich Hinweise auf „Korruptionsprävention" durch bessere Planung, Reduktion von Missmanagement und Erhöhung von Nachkontrollen als Folge erhöhter Transparenz.

Frage 6: Welche öffentlichen Einkaufsdaten kennen Sie und wie werden diese Ihrer Erfahrung nach heute veröffentlicht?

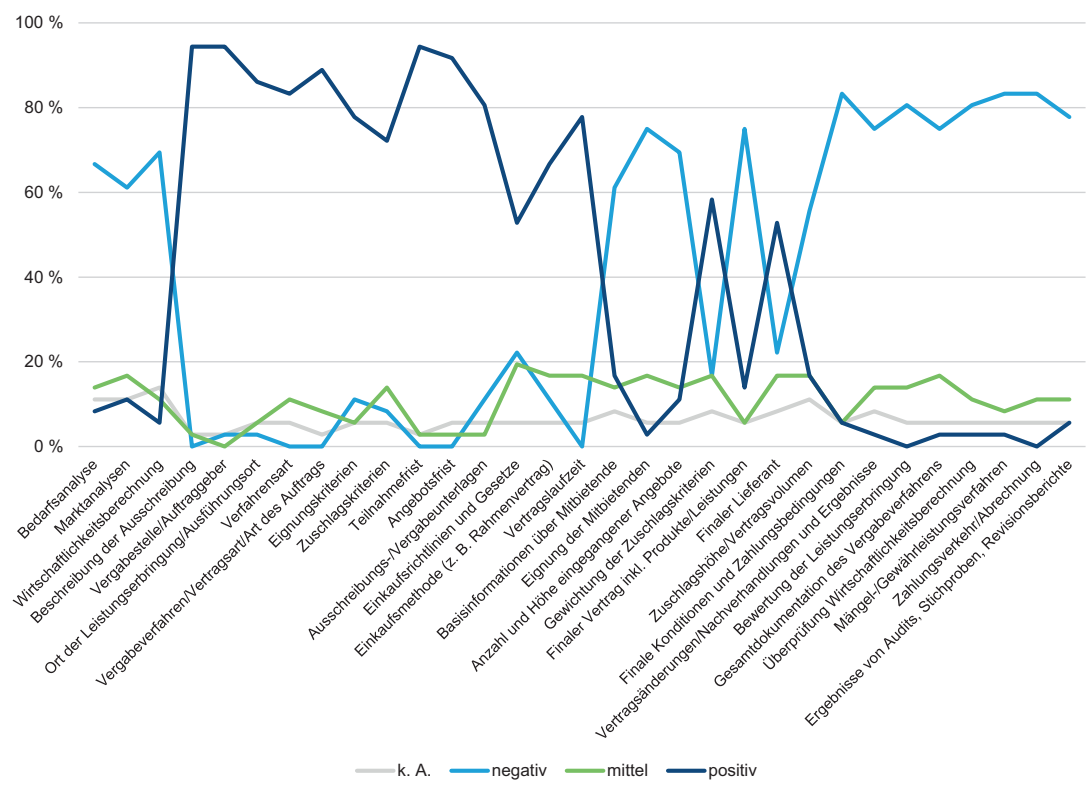

Abbildung 8.6 Aktuelle Veröffentlichung öffentlicher Einkaufsdaten im Unterschwellenbereich

Tabelle 8.18 Aktuelle Veröffentlichung öffentlicher Einkaufsdaten im Unterschwellenbereich

\begin{tabular}{l|c|c|c|c|c|c|c|c}
\hline Antworten & \multicolumn{2}{|c|}{ Positiv } & \multicolumn{2}{c|}{ Mittel } & \multicolumn{2}{c|}{ Negativ } & \multicolumn{2}{c}{ k. A. } \\
\hline & $\%$ & Anz. & $\%$ & Anz. & $\%$ & Anz. & $\%$ & Anz. \\
\hline Planung & & & & & & & & \\
\hline Bedarfsanalyse & $8 \%$ & 3 & $14 \%$ & 5 & $67 \%$ & 24 & $11 \%$ & 4 \\
\hline Marktanalysen & $11 \%$ & 4 & $17 \%$ & 6 & $61 \%$ & 22 & $11 \%$ & 4 \\
\hline
\end{tabular}


Tabelle 8.18 (Fortsetzung)

\begin{tabular}{|c|c|c|c|c|c|c|c|c|}
\hline \multirow[t]{2}{*}{ Antworten } & \multicolumn{2}{|c|}{ Positiv } & \multicolumn{2}{|c|}{ Mittel } & \multicolumn{2}{|c|}{ Negativ } & \multicolumn{2}{|c|}{ k. A. } \\
\hline & $\%$ & Anz. & $\%$ & Anz. & $\%$ & Anz. & $\%$ & Anz. \\
\hline $\begin{array}{l}\text { Wirtschaftlichkeits- } \\
\text { berechnung }\end{array}$ & $6 \%$ & 2 & $11 \%$ & 4 & $69 \%$ & 25 & $14 \%$ & 5 \\
\hline Mittelwert & $8 \%$ & $\%$ & $14 \%$ &.$\%$ & $66 \%$ &.$\%$ & $12 \%$ &.$\%$ \\
\hline \multicolumn{9}{|l|}{ Ausschreibung } \\
\hline $\begin{array}{l}\text { Beschreibung der } \\
\text { Ausschreibung }\end{array}$ & $94 \%$ & 34 & $3 \%$ & 1 & $0 \%$ & 0 & $3 \%$ & 1 \\
\hline $\begin{array}{l}\text { Vergabestelle/ } \\
\text { Auftraggeber }\end{array}$ & $94 \%$ & 34 & $0 \%$ & 0 & $3 \%$ & 1 & $3 \%$ & 1 \\
\hline $\begin{array}{l}\text { Ort der Leistungser- } \\
\text { bringung }\end{array}$ & $86 \%$ & 31 & $6 \%$ & 2 & $3 \%$ & 1 & $6 \%$ & 2 \\
\hline Verfahrensart & $83 \%$ & 30 & $11 \%$ & 4 & $0 \%$ & 0 & $6 \%$ & 2 \\
\hline $\begin{array}{l}\text { Vergabeverfahren/ } \\
\text { Vertragsart }\end{array}$ & $89 \%$ & 32 & $8 \%$ & 3 & $0 \%$ & 0 & $3 \%$ & 1 \\
\hline Eignungskriterien & $78 \%$ & 28 & $6 \%$ & 2 & $11 \%$ & 4 & $6 \%$ & 2 \\
\hline Zuschlagskriterien & $72 \%$ & 26 & $14 \%$ & 5 & $8 \%$ & 3 & $6 \%$ & 2 \\
\hline Teilnahmefrist & $94 \%$ & 34 & $3 \%$ & 1 & $0 \%$ & 0 & $3 \%$ & 1 \\
\hline Angebotsfrist & $92 \%$ & 33 & $3 \%$ & 1 & $0 \%$ & 0 & $6 \%$ & 2 \\
\hline $\begin{array}{l}\text { Ausschreibungsunter- } \\
\text { lagen }\end{array}$ & $81 \%$ & 29 & $3 \%$ & 1 & $11 \%$ & 4 & $6 \%$ & 2 \\
\hline $\begin{array}{l}\text { Einkaufsrichtlinien } \\
\text { und Gesetze }\end{array}$ & $53 \%$ & 19 & $19 \%$ & 7 & $22 \%$ & 8 & $6 \%$ & 2 \\
\hline Einkaufsmethode & $67 \%$ & 24 & $17 \%$ & 6 & $11 \%$ & 4 & $6 \%$ & 2 \\
\hline Vertragslaufzeit & $78 \%$ & 28 & $17 \%$ & 6 & $0 \%$ & 0 & $6 \%$ & 2 \\
\hline $\begin{array}{l}\text { Basisinformationen } \\
\text { Mitbietende }\end{array}$ & $17 \%$ & 6 & $14 \%$ & 5 & $61 \%$ & 22 & $8 \%$ & 3 \\
\hline Mittelwert & $77 \%$ &.$\%$ & $9 \%$ &.$\%$ & $9 \%$ &.$\%$ & $5 \%$ &.$\%$ \\
\hline \multicolumn{9}{|l|}{ Bewertung } \\
\hline $\begin{array}{l}\text { Eignung der } \\
\text { Mitbietenden }\end{array}$ & $3 \%$ & 1 & $17 \%$ & 6 & $75 \%$ & 27 & $6 \%$ & 2 \\
\hline $\begin{array}{l}\text { Anzahl/Höhe eingeg. } \\
\text { Angebote }\end{array}$ & $11 \%$ & 4 & $14 \%$ & 5 & $69 \%$ & 25 & $6 \%$ & 2 \\
\hline $\begin{array}{l}\text { Gewichtung der } \\
\text { Zuschlagskriterien }\end{array}$ & $58 \%$ & 21 & $17 \%$ & 6 & $17 \%$ & 6 & $8 \%$ & 3 \\
\hline Mittelwert & $24 \%$ &.$\%$ & $16 \%$ &. & $54 \%$ &.$\%$ & $7 \%$ &.$\%$ \\
\hline
\end{tabular}


Tabelle 8.18 (Fortsetzung)

\begin{tabular}{|c|c|c|c|c|c|c|c|c|}
\hline \multirow{2}{*}{ Antworten } & \multicolumn{2}{|c|}{ Positiv } & \multicolumn{2}{|c|}{ Mittel } & \multicolumn{2}{|c|}{ Negativ } & \multicolumn{2}{|c|}{ k. A. } \\
\hline & $\%$ & Anz. & $\%$ & Anz. & $\%$ & Anz. & $\%$ & Anz. \\
\hline \multicolumn{9}{|l|}{ Vergabe } \\
\hline Finaler Vertrag & $14 \%$ & 5 & $6 \%$ & 2 & $75 \%$ & 27 & $6 \%$ & 2 \\
\hline Finaler Lieferant & $53 \%$ & 19 & $17 \%$ & 6 & $22 \%$ & 8 & $8 \%$ & 3 \\
\hline $\begin{array}{l}\text { Zuschlagshöhe/ } \\
\text { Vertragsvolumen }\end{array}$ & $17 \%$ & 6 & $17 \%$ & 6 & $56 \%$ & 20 & $11 \%$ & 4 \\
\hline $\begin{array}{l}\text { Finale Konditionen/ } \\
\text { Zahlungsbed. }\end{array}$ & $6 \%$ & 2 & $6 \%$ & 2 & $83 \%$ & 30 & $6 \%$ & 2 \\
\hline Mittelwert & $22 \%$ &.$\%$ & $11 \%$ &.$\%$ & $59 \%$ &.$\%$ & $8 \%$ &. \\
\hline \multicolumn{9}{|l|}{ Ausführung } \\
\hline $\begin{array}{l}\text { Vertragsänderungen \& } \\
\text { Ergebnisse }\end{array}$ & $3 \%$ & 1 & $14 \%$ & 5 & $75 \%$ & 27 & $8 \%$ & 3 \\
\hline $\begin{array}{l}\text { Bewertung der } \\
\text { Leistungserbringung }\end{array}$ & $0 \%$ & 0 & $14 \%$ & 5 & $81 \%$ & 29 & $6 \%$ & 2 \\
\hline Gesamtdokumentation & $3 \%$ & 1 & $17 \%$ & 6 & $75 \%$ & 27 & $6 \%$ & 2 \\
\hline $\begin{array}{l}\text { Überprüfung } \\
\text { Business Case }\end{array}$ & $3 \%$ & 1 & $11 \%$ & 4 & $81 \%$ & 29 & $6 \%$ & 2 \\
\hline $\begin{array}{l}\text { Mängel-/Gewähr- } \\
\text { leistungsverfahren }\end{array}$ & $3 \%$ & 1 & $8 \%$ & 3 & $83 \%$ & 30 & $6 \%$ & 2 \\
\hline $\begin{array}{l}\text { Zahlungsverkehr/ } \\
\text { Abrechnung }\end{array}$ & $0 \%$ & 0 & $11 \%$ & 4 & $83 \%$ & 30 & $6 \%$ & 2 \\
\hline $\begin{array}{l}\text { Ergebnisse von } \\
\text { Audits, Stichproben }\end{array}$ & $6 \%$ & 2 & $11 \%$ & 4 & $78 \%$ & 28 & $6 \%$ & 2 \\
\hline Mittelwert & $2 \%$ &. & $12 \%$ &.$\%$ & $79 \%$ &.$\%$ & $6 \%$ &.$\%$ \\
\hline Summe &.$\%$ & 461 &.$\%$ & 123 &.$\%$ & 461 &. & 71 \\
\hline Mittelwert Gesamt & $41 \%$ & 15 & $11 \%$ & 4 & $41 \%$ & 15 & $6 \%$ & 2 \\
\hline
\end{tabular}

\section{Bewertung:}

Auf die Frage, welche öffentlichen Einkaufsdaten die Teilnehmer bereits kennen und wie diese heute veröffentlicht werden, bestätigen dies mit im Mittel nur $8 \%$ der Befragten für den Teilprozess der Planung. Mit $77 \%$ erfährt der Teilprozess der Ausschreibung die höchste Zustimmung. Die Teilprozesse Bewertung und Vergabe liegen mit im Mittel $24 \%$ respektive $22 \%$ auf ähnlichem Zustimmungsniveau. Am Geringsten fällt die Bestätigung mit $2 \%$ für den 
Teilprozess der Ausführung aus. Damit sind überwiegende Teile des öffentlichen Gesamtbeschaffungsprozesses für Dritte nicht zugänglich, wie Abbildung 8.6/ Tabelle 8.18 aufzeigt.

Die Ergebnisse bestätigen insgesamt die Resultate der Portalanalyse in Abschnitt 7.3.9.5. Besonders interessant ist, dass auch hier der „,finale Lieferant“ von nur $53 \%$ als bereits heute veröffentlichte Information angegeben wird obwohl dieser in Vergabebekanntmachungen verpflichtend zu nennen ist. Auch dies entspricht den Ergebnissen der Portalanalyse, nach welcher die Angabe des finalen Lieferanten als einzige Angabe hinter den juristischen Vorgaben deutlich zurückbleibt (siehe Abschnitt 7.3.9.4.4).

\section{Frage 7: Wie werden öffentliche Einkaufsdaten Ihrer Erfahrung nach heute} überwiegend in den Verwaltungen gespeichert?

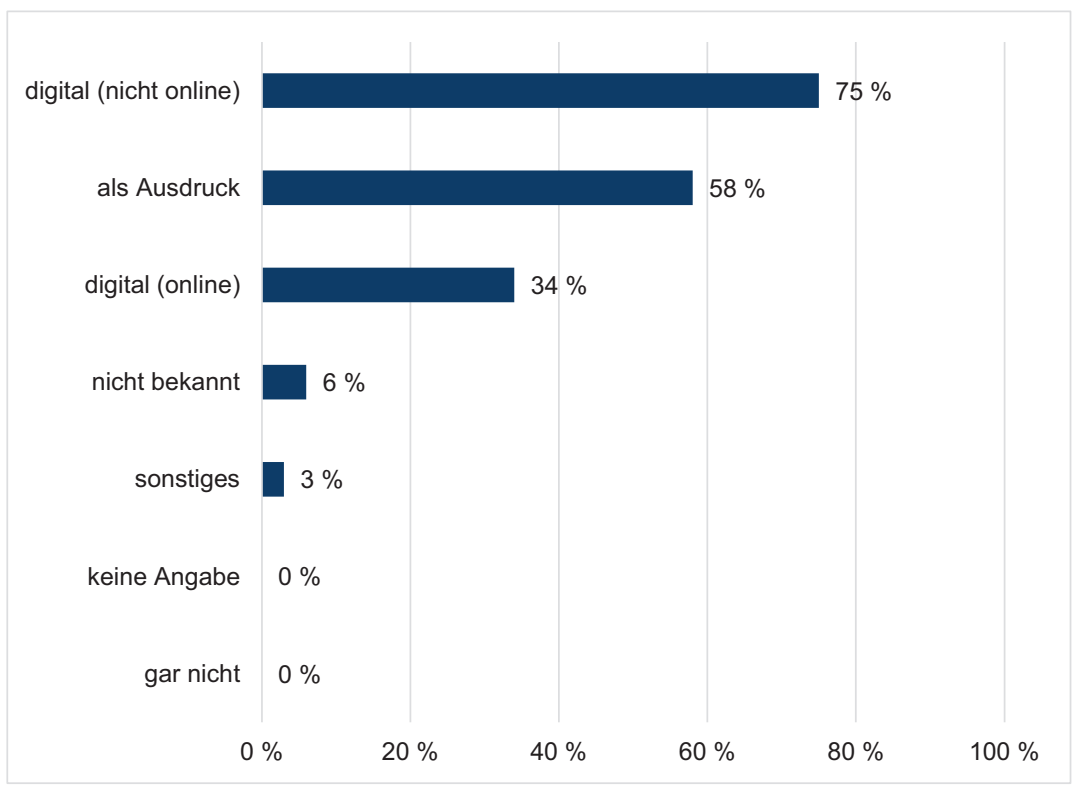

Abbildung 8.7 Aktuelle Art der Datenspeicherung öffentlicher Einkaufsdaten 
Tabelle 8.19 Aktuelle Art der Datenspeicherung nach Antworthäufigkeit

\begin{tabular}{l|c|c}
\hline Antworten & Prozent & Anzahl \\
\hline digital (nicht online) & $75 \%$ & 27 \\
\hline als Ausdruck & $58 \%$ & 21 \\
\hline digital (online) & $34 \%$ & 12 \\
\hline nicht bekannt & $6 \%$ & 2 \\
\hline sonstige & $3 \%$ & 1 \\
\hline gar nicht & $0 \%$ & 0 \\
\hline keine Angabe & $0 \%$ & $\mathbf{6 3}$ \\
\hline Summe &.$\%$ & $\mathbf{9}$ \\
\hline Mittelwert & $\mathbf{2 5 \%}$ & \\
\hline
\end{tabular}

Tabelle 8.20 Aktuelle Art der Datenspeicherung nach Antwortreihenfolge

\begin{tabular}{l|c|c}
\hline Antworten & Prozent & Anzahl \\
\hline als Ausdruck & $58 \%$ & 21 \\
\hline digital (nicht online) & $75 \%$ & 27 \\
\hline digital (online) & $34 \%$ & 0 \\
\hline gar nicht & $0 \%$ & 2 \\
\hline nicht bekannt & $6 \%$ & 1 \\
\hline sonstige & $3 \%$ & 0 \\
\hline keine Angabe & $0 \%$ & $\mathbf{6 3}$ \\
\hline Summe &.$/$ & $\mathbf{9}$ \\
\hline Mittelwert & $\mathbf{2 5 \%}$ & 0 \\
\hline
\end{tabular}

\section{Bewertung:}

Auf die Frage (Mehrfachauswahl), wie öffentliche Einkaufsdaten heute überwiegend gespeichert werden, antwortet gemäß Abbildung 8.7/Tabelle 8.19/ Tabelle 8.20 eine Mehrheit von $75 \%$, dass dies digital (nicht online) erfolgt. Die Digital- und Online-Datenspeicherung wird von $34 \%$ der Befragten bestätigt. Als Ausdruck werden noch $58 \%$ der Daten vorgehalten. Hier besteht deutlich Veränderungspotential zur digitalen Ablage. 
Frage 8: Sofern eine elektronische Speicherung der Daten bereits erfolgt: In welchen Formaten werden öffentliche Einkaufsdaten überwiegend gespeichert?

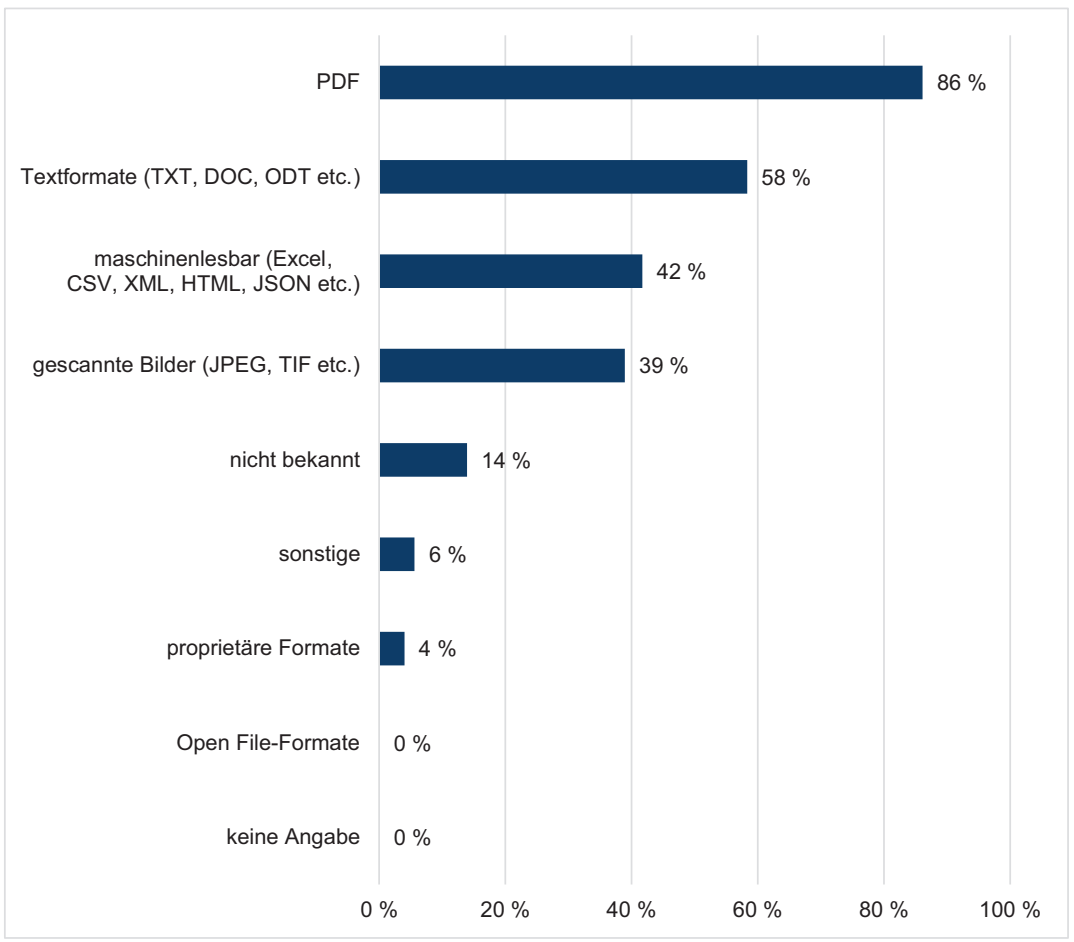

Abbildung 8.8 Aktuelle Formate der Speicherung öffentlicher Einkaufsdaten 
Tabelle 8.21 Aktuelle Formate der Speicherung öffentlicher Einkaufsdaten

\begin{tabular}{l|c|c}
\hline Antworten & Prozent & Anzahl \\
\hline PDF & $86 \%$ & 31 \\
\hline Textformate (TXT, DOC, ODT etc.) & $58 \%$ & 21 \\
\hline maschinenlesbar (Excel, CSV, & $42 \%$ & 15 \\
XML, HTML, JSON etc.) & & 14 \\
\hline gescannte Bilder (JPEG, TIF etc.) & $39 \%$ & 5 \\
\hline nicht bekannt & $14 \%$ & 2 \\
\hline sonstige & $6 \%$ & 1 \\
\hline proprietäre Formate & $4 \%$ & 0 \\
\hline Open File-Formate & $0 \%$ & 0 \\
\hline keine Angabe & $0 \%$ & $\mathbf{8 9}$ \\
\hline Summe &.$\%$ & $\mathbf{1 0}$ \\
\hline Mittelwert & $\mathbf{2 8 \%}$ & \\
\hline
\end{tabular}

\section{Bewertung:}

Auf die Frage (Mehrfachauswahl), in welchen Formaten öffentliche Einkaufsdaten heute überwiegend gespeichert werden, bejahen $86 \%$ der Teilnehmer PDF. Textformate werden von $58 \%$ und Bildformate von $39 \%$ der Teilnehmer genannt. In maschinenlesbaren Formaten werden $42 \%$ der Daten bereitgehalten, für Open File-Formate gibt es gar keine Nennungen (0\%) (Abbildung 8.8/ Tabelle 8.21).

Unter „sonstige“ wird noch aufgeführt, dass immer häufiger ZIP-Dateien verfügbar sind, sofern die Daten aus einer eVergabe stammen und ebenfalls strukturiert im Rahmen einer elektronischen Vergabeakte innerhalb der Vergabemanagementsysteme.

Hieran zeigt sich einerseits die Notwendigkeit, den maschinenlesbaren Anteil auszubauen, sofern man die Öffnung öffentlicher Einkaufsdaten unterstützt. Andererseits wird der indirekte Nutzen aus der eVergabe ersichtlich: Durch die Verpflichtung zur elektronischen Abwicklung der Ausschreibung werden öffentliche Einkaufsdaten zunehmend elektronisch verfügbar. 
Frage 9: Wo werden die von Ihnen benannten Einkaufsdaten heute veröffentlicht?

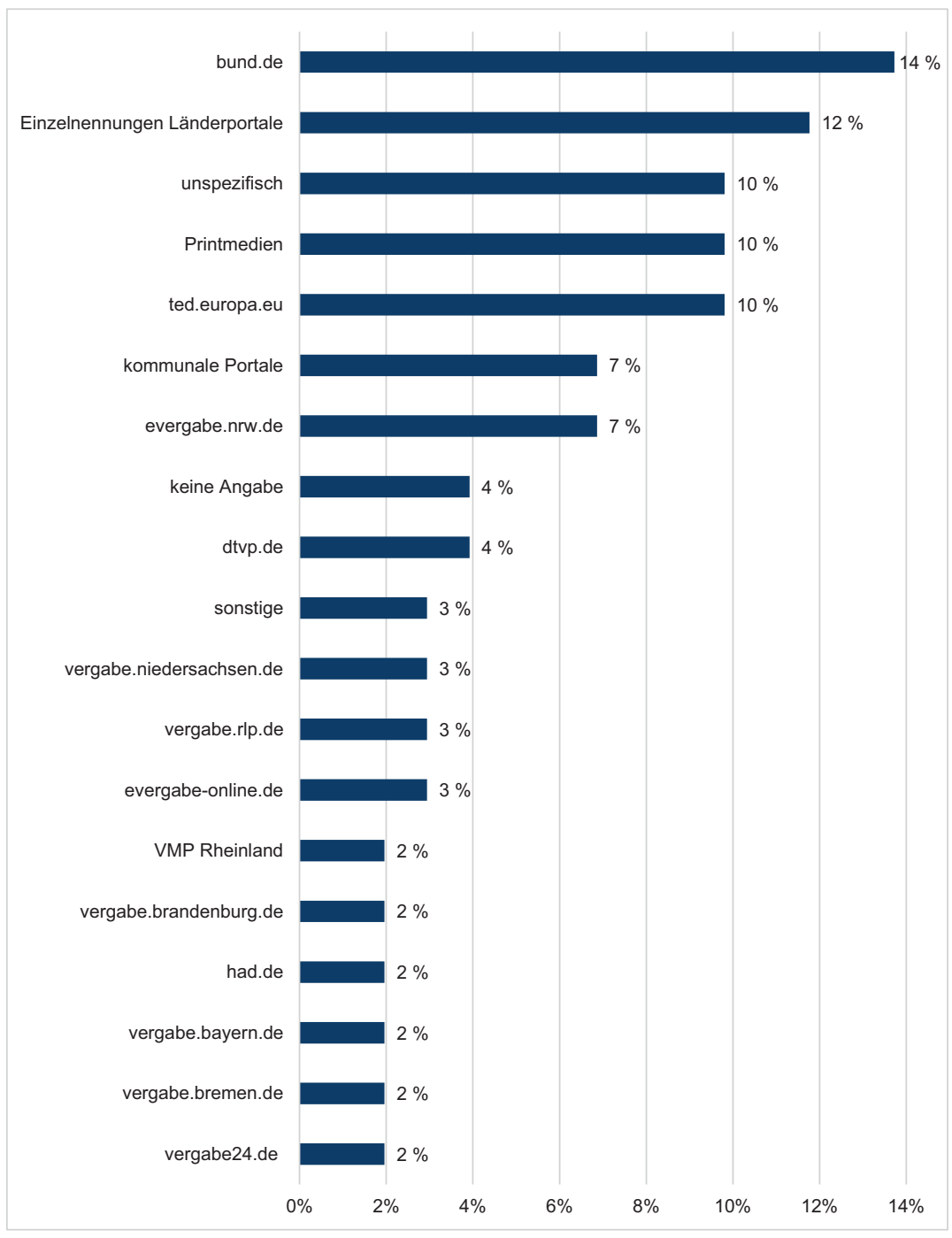

Abbildung 8.9 Aktuelle Orte der Bereitstellung öffentlicher Einkaufsdaten 


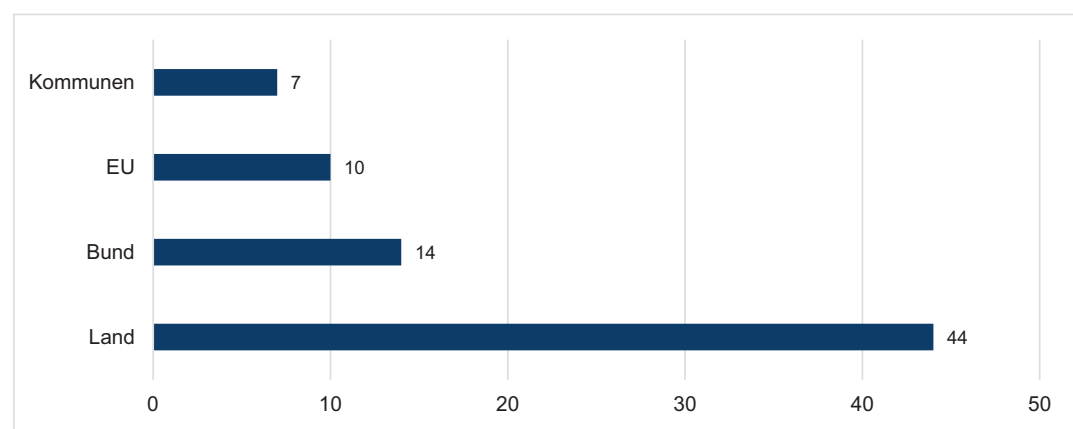

Abbildung 8.10 Aktuelle Bereitstellung öffentlicher Einkaufsdaten nach Kategorien

Tabelle 8.22 Aktuelle Orte der Bereitstellung öffentlicher Einkaufsdaten

\begin{tabular}{l|c|c}
\hline Antworten & Prozent & Anzahl \\
\hline bund.de & $14 \%$ & 14 \\
\hline Einzelnennungen Länderportale & $12 \%$ & 12 \\
\hline ted.europa.eu & $10 \%$ & 10 \\
\hline Printmedien & $10 \%$ & 10 \\
\hline unspezifisch & $10 \%$ & 10 \\
\hline evergabe.nrw.de & $7 \%$ & 7 \\
\hline kommunale Portale & $7 \%$ & 7 \\
\hline dtvp.de & $4 \%$ & 4 \\
\hline keine Angabe & $4 \%$ & 4 \\
\hline evergabe-online.de & $3 \%$ & 3 \\
\hline vergabe.rlp.de & $3 \%$ & 3 \\
\hline vergabe.niedersachsen.de & $3 \%$ & 3 \\
\hline sonstige & $3 \%$ & 3 \\
\hline vergabe24.de & $2 \%$ & 2 \\
\hline vergabe.bremen.de & $2 \%$ & 2 \\
\hline vergabe.bayern.de & $2 \%$ & 2 \\
\hline
\end{tabular}

(Fortsetzung) 
Tabelle 8.22 (Fortsetzung)

\begin{tabular}{l|c|c}
\hline Antworten & Prozent & Anzahl \\
\hline had.de & $2 \%$ & 2 \\
\hline vergabe.brandenburg.de & $2 \%$ & 2 \\
\hline $\begin{array}{l}\text { VMP (Vergabemanagementportal) } \\
\text { Rheinland }\end{array}$ & $2 \%$ & 2 \\
\hline Summe & $\mathbf{1 0 0 \%}$ & $\mathbf{1 0 2}$ \\
\hline
\end{tabular}

\section{Bewertung:}

Auf die Frage (offene Nennungen), wo die benannten öffentlichen Einkaufsdaten veröffentlicht werden, wird mit $14 \%$ das Portal https://service.bund.de genannt, gefolgt von „Einzelnennungen für Länderportale“ mit $12 \%$ (Abbildung 8.9/ Abbildung 8.10/Tabelle 8.22). Hierunter fallen einmalige Nennungen von Portalen der Länder wie beispielsweise https://www.deutsche-evergabe.de. Auf die Nutzung von Printmedien und https://ted.europa.eu entfallen jeweils $10 \%$ der Nennungen. Zu Printmedien zählen beispielsweise das Amtsblatt der europäischen Union, Tageszeitungen, der Bundesanzeiger oder diverse Printmedien. Unter „,kommunale Portale“ (7 \% der Nennungen) wurden unter anderem die Vergabeseiten der Städte Köln, Bonn und Wuppertal sowie Vergabemarktplätze der jeweiligen Kommunen angegeben. ${ }^{2}$

Wenn man die Ergebnisse nochmals nach EU, Bund, Land und Kommunen gruppiert, wie in Abbildung 8.10 geschehen, wird deutlich, dass der größte Anteil der Nennungen auf Länderportale entfällt. Insgesamt bestätigen die Antworten, dass es in Deutschland eine stark dezentrale Portalstruktur gibt, was mit der Portalanalyse in Abschnitt 7.3.9 konform geht.

${ }^{2}$ https://www.stadt-koeln.de/wirtschaft/vergabe/aktuelle-auftraege $? \mathrm{id}=68785 \&$ mode=detail; https://opendata.bonn.de/dataset/ausschreibungen-vergabeverfahren; http://web-application. wuppertal.de/online-vvp/info_bekanntmachung_neu.php. 
Frage 10: Welche Anwendungen (unter anderem Datenbanken, Software, Apps), Berichte und Visualisierungen, die auf öffentlichen Einkaufsdaten beruhen, kennen Sie bereits?

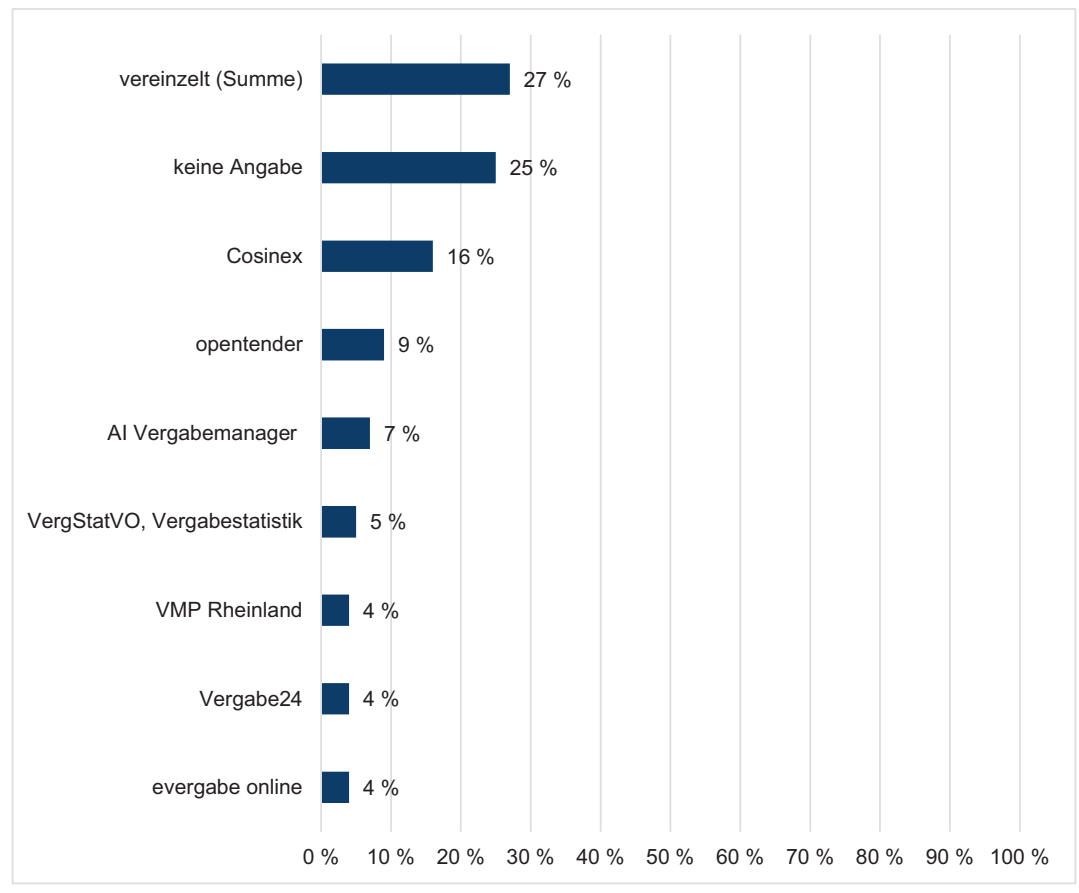

Abbildung 8.11 Aktuelle Anwendungen öffentlicher Einkaufsdaten

Tabelle 8.23 Aktuelle Anwendungen öffentlicher Einkaufsdaten

\begin{tabular}{l|c|c}
\hline Antworten & Prozent & Anzahl \\
\hline keine Angabe & $25 \%$ & 14 \\
\hline Cosinex GmbH & $16 \%$ & 9 \\
\hline opentender & $9 \%$ & 5 \\
\hline AI Vergabemanager & $7 \%$ & 4 \\
\hline VergStatVO, Vergabestatistik & $5 \%$ & 3 \\
\hline evergabe online & $4 \%$ & 2 \\
\hline Vergabe24 & $4 \%$ & 2 \\
\hline VMP (Vergabemanagementportal) & $4 \%$ & 2 \\
Rheinland & & \\
\hline
\end{tabular}


Tabelle 8.23 (Fortsetzung)

\begin{tabular}{l|c|c}
\hline Antworten & Prozent & Anzahl \\
\hline ava sign & $2 \%$ & 1 \\
\hline B_I Medien GmbH & $2 \%$ & 1 \\
\hline Bund & $2 \%$ & 1 \\
\hline Decision Advisor/Softguide & $2 \%$ & 1 \\
\hline Deutsches Ausschreibungsblatt & $2 \%$ & 1 \\
\hline EU SIMAP & $2 \%$ & 1 \\
\hline evergabe NRW & $2 \%$ & 1 \\
\hline Heinze Baudatenbank & $2 \%$ & 1 \\
\hline Open Contracting Partnership & $2 \%$ & 1 \\
\hline Portale der Länder & $2 \%$ & 1 \\
\hline RIB Software AG & $2 \%$ & 1 \\
\hline Staatsanzeiger Baden-Württemberg & $2 \%$ & 1 \\
\hline subreport Verlag Schawe GmbH & $2 \%$ & 1 \\
\hline TED Portal & $2 \%$ & 1 \\
\hline VERIS & $\mathbf{1 0 0} \%$ & $\mathbf{5 6}$ \\
\hline Summe & $\mathbf{4 \%}$ & $\mathbf{2}$ \\
\hline Mittelwert & & 1 \\
\hline
\end{tabular}

\section{Bewertung:}

Die Antworten auf die Frage (offene Nennungen), welche Anwendungen (unter anderem Datenbanken, Software, Apps), Berichte und Visualisierungen, die auf öffentlichen Einkaufsdaten beruhen, die Teilnehmer bereits kennen, wurden gemäß Abbildung 8.11/Tabelle 8.23 gruppiert. Demnach entfallen $27 \%$ auf Einzelnennungen. $\mathrm{Zu}$ diesen zählen beispielsweise die B_I Medien GmbH, https://www. evergabe.nrw.de, EU SIMAP (https://simap.ted.europa.eu/de), die OCP, Portale der Länder, subreport Verlag Schawe $\mathrm{GmbH}$, die Portale https://ted.europa.eu und https://service.bund.de. Das macht sowohl die Vielfalt der vorhandenen Anbieter und Lösungen als auch abermals die starke Marktfragmentierung ersichtlich: Aufgrund der nicht vorhandenen Konsolidierung besteht hier noch erhebliches Marktgestaltungspotential für Anbieter im Umfeld öffentlicher Einkaufsdaten. Dieses Potential würde sich durch die Öffnung öffentlicher Einkaufsdaten nochmals erhöhen.

Der Anteil der Antwort für „keine Angabe“ ist mit $25 \%$ recht hoch. Dies könnte dafür sprechen, dass es nicht den einen bekannten Anbieter beziehungsweise die eine etablierte Lösung gibt. Es folgen mit $16 \%$ die Cosinex $\mathrm{GmbH}$, mit $9 \%$ https://opentender.eu/ und $7 \%$ der Vergabemanager der Administration Intelligence AG (AI-Vergabemanager). 
Frage 11: Was behindert aktuell die Freigabe öffentlicher Einkaufsdaten am meisten?

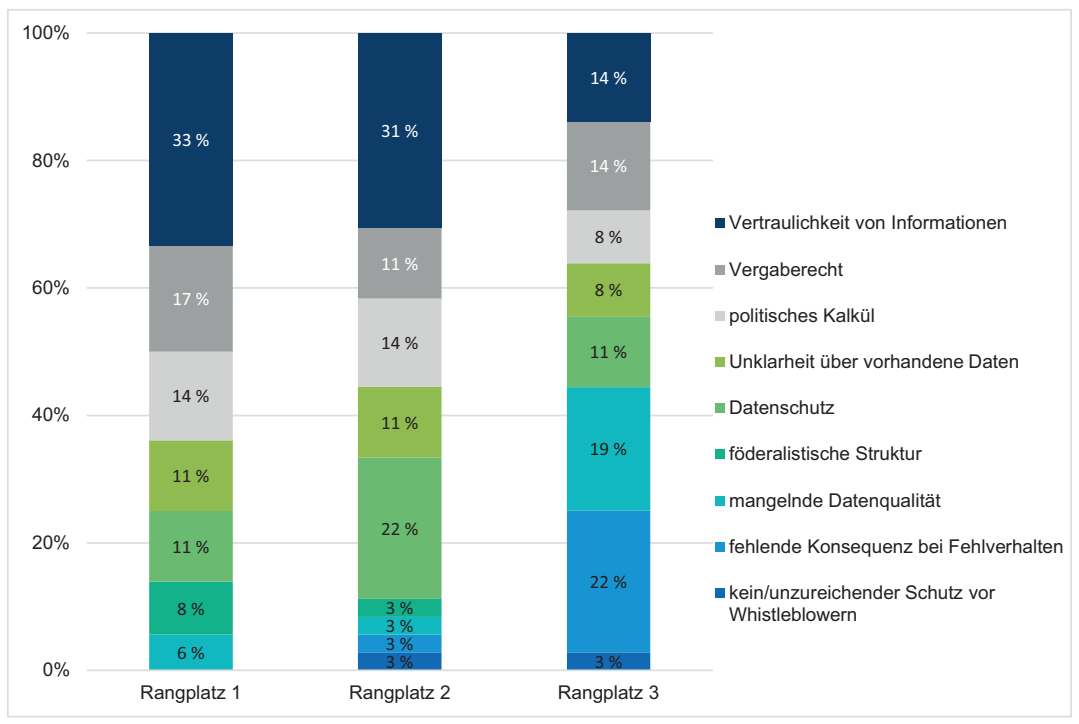

Abbildung 8.12 Hindernisse der Öffnung öffentlicher Einkaufsdaten

Tabelle 8.24 Hindernisse der Öffnung öffentlicher Einkaufsdaten

\begin{tabular}{|c|c|c|c|c|c|c|c|}
\hline \multirow[t]{2}{*}{ Antworten } & \multicolumn{2}{|c|}{ Rangplatz 1} & \multicolumn{2}{|c|}{ Rangplatz 2} & \multicolumn{2}{|c|}{ Rangplatz 3} & \multirow[b]{2}{*}{$\sum$} \\
\hline & $\%$ & Anz. & $\%$ & Anz. & $\%$ & Anz. & \\
\hline $\begin{array}{l}\text { Vertraulichkeit von } \\
\text { Informationen }\end{array}$ & $33 \%$ & 12 & $31 \%$ & 11 & $14 \%$ & 5 & $78 \%$ \\
\hline Vergaberecht & $17 \%$ & 6 & $11 \%$ & 4 & $14 \%$ & 5 & $42 \%$ \\
\hline politisches Kalkül & $14 \%$ & 5 & $14 \%$ & 5 & $8 \%$ & 3 & $36 \%$ \\
\hline Datenschutz & $11 \%$ & 4 & $22 \%$ & 8 & $11 \%$ & 4 & $44 \%$ \\
\hline $\begin{array}{l}\text { Unklarheit über } \\
\text { vorhandene Daten }\end{array}$ & $11 \%$ & 4 & $11 \%$ & 4 & $8 \%$ & 3 & $30 \%$ \\
\hline föderalistische Struktur & $8 \%$ & 3 & $3 \%$ & 1 & $0 \%$ & 0 & $11 \%$ \\
\hline mangelnde Datenqualität & $6 \%$ & 2 & $3 \%$ & 1 & $19 \%$ & 7 & $28 \%$ \\
\hline $\begin{array}{l}\text { unzureichender Schutz vor } \\
\text { Whistleblowern }\end{array}$ & $0 \%$ & 0 & $3 \%$ & 1 & $3 \%$ & 1 & $6 \%$ \\
\hline
\end{tabular}


Tabelle 8.24 (Fortsetzung)

\begin{tabular}{l|c|c|c|c|c|c|c}
\hline \multirow{2}{*}{ Antworten } & \multicolumn{2}{|c|}{ Rangplatz 1 } & \multicolumn{2}{|c|}{ Rangplatz 2 } & \multicolumn{2}{c|}{ Rangplatz 3 } & \multirow{2}{*}{} \\
\cline { 2 - 8 } & $\%$ & Anz. & $\%$ & Anz. & $\%$ & Anz. & $\sum$ \\
\hline $\begin{array}{l}\text { fehlende Konsequenz bei } \\
\text { Fehlverhalten }\end{array}$ & $0 \%$ & 0 & $3 \%$ & 1 & $22 \%$ & 8 & $25 \%$ \\
\hline Summe &. & 36 &. & 36 &. & $\mathbf{3 6}$ &. \\
\hline Mittelwert &. &. &. &. &. &. & $\mathbf{3 3} \%$ \\
\hline
\end{tabular}

\section{Bewertung:}

Als wesentliches Hindernis zur Öffnung der öffentlichen Einkaufsdaten wird gemäß Abbildung 8.12/Tabelle 8.24 an erster Stelle (Summe über alle Rangplätze) mit $78 \%$ der Antworten die Vertraulichkeit der Informationen gesehen. Mit deutlichem Abstand folgt mit $44 \%$ der Datenschutz sowie mit $42 \%$ das Vergaberecht. An vierter Stelle steht mit immerhin $36 \%$ politisches Kalkül. Mit nur $6 \%$ wird ein unzureichender Schutz vor Hinweisgebern/Whistleblowern als Hindernis eingestuft und mit $11 \%$ die föderalistische Struktur. Dies unterstreicht die Erkenntnisse der Literaturrecherche, die bereits den Klärungsbedarf zu Betriebs- und Geschäftsgeheimnissen aufgezeigt hat (siehe Abschnitt 7.1.3).

\section{Frage 12: Welche wesentlichen Voraussetzungen müssten noch geschaffen werden, um die Öffnung öffentlicher Einkaufsdaten voranzutreiben?}

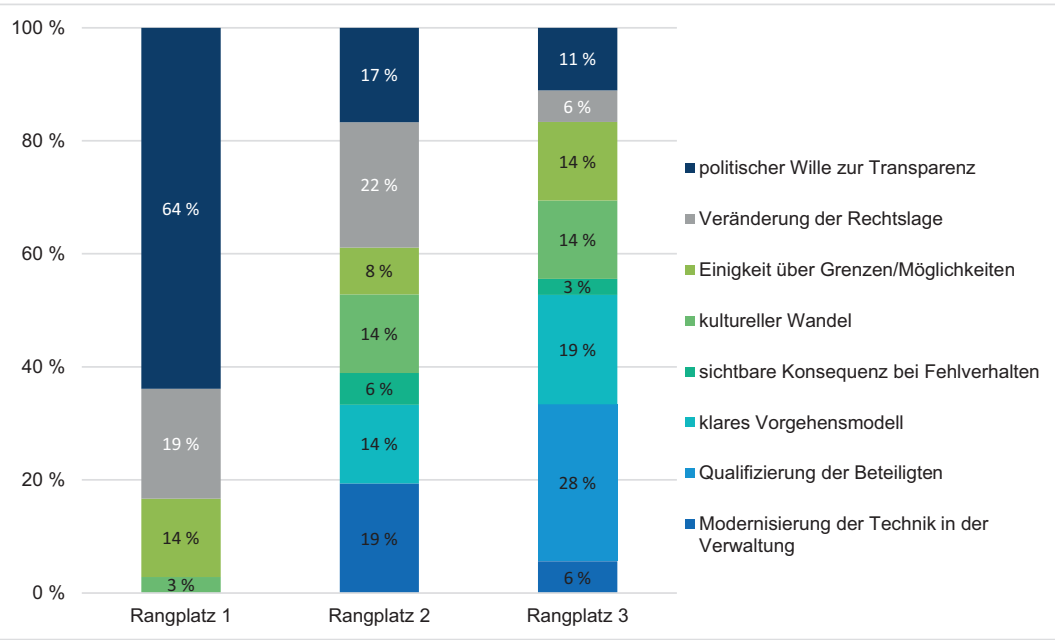

Abbildung 8.13 Voraussetzungen der Öffnung öffentlicher Einkaufsdaten 
Tabelle 8.25 Voraussetzungen der Öffnung öffentlicher Einkaufsdaten

\begin{tabular}{|c|c|c|c|c|c|c|c|}
\hline \multirow[t]{2}{*}{ Antworten } & \multicolumn{2}{|c|}{ Rangplatz 1} & \multicolumn{2}{|c|}{ Rangplatz 2} & \multicolumn{2}{|c|}{ Rangplatz 3} & \multirow[b]{2}{*}{$\sum$} \\
\hline & $\%$ & Anz. & $\%$ & Anz. & $\%$ & Anz. & \\
\hline $\begin{array}{l}\text { politischer Wille zur } \\
\text { Transparenz }\end{array}$ & $64 \%$ & 23 & $17 \%$ & 6 & $11 \%$ & 4 & $92 \%$ \\
\hline Veränderung der Rechtslage & $19 \%$ & 7 & $22 \%$ & 8 & $6 \%$ & 2 & $47 \%$ \\
\hline $\begin{array}{l}\text { Einigkeit über Grenzen/ } \\
\text { Möglichkeiten }\end{array}$ & $14 \%$ & 5 & $8 \%$ & 3 & $14 \%$ & 5 & $36 \%$ \\
\hline kultureller Wandel & $3 \%$ & 1 & $14 \%$ & 5 & $14 \%$ & 5 & $31 \%$ \\
\hline $\begin{array}{l}\text { Modernisierung der Ver- } \\
\text { waltungs-Technik }\end{array}$ & $0 \%$ & 0 & $19 \%$ & 7 & $6 \%$ & 2 & $25 \%$ \\
\hline Qualifizierung der Beteiligten & $0 \%$ & 0 & $0 \%$ & 0 & $28 \%$ & 10 & $28 \%$ \\
\hline klares Vorgehensmodell & $0 \%$ & 0 & $14 \%$ & 5 & $19 \%$ & 7 & $33 \%$ \\
\hline $\begin{array}{l}\text { sichtbare Konsequenz bei } \\
\text { Fehlverhalten }\end{array}$ & $0 \%$ & 0 & $6 \%$ & 2 & $3 \%$ & 1 & $9 \%$ \\
\hline Summe &.$\%$ & 36 &.$\%$ & 36 &.$\%$ & 36 &.$\%$ \\
\hline Mittelwert &.$\%$ &.$\%$ &.$\%$ &.$\%$ &.$\%$ &.$\%$ & $37 \%$ \\
\hline
\end{tabular}

\section{Bewertung:}

Als weitaus wichtigste Voraussetzung zur Öffnung der öffentlichen Einkaufsdaten wird der politische Wille zur Transparenz gesehen. Sie nimmt laut Abbildung 8.13/Tabelle 8.25 den ersten Platz ein (Summe über alle Rangplätze), und zwar mit einem Wert von $92 \%$ der Antworten. Mit erheblichem Abstand folgt mit $47 \%$ die Forderung nach einer Veränderung der Rechtslage. An dritter Stelle steht die Einigkeit über Grenzen/Möglichkeiten mit 36\%. Mit nur 9\% wird eine sichtbare Konsequenz bei Fehlverhalten als erforderliche Voraussetzung eingestuft. 


\subsubsection{Künftige Situation}

Frage 13: Inwieweit stimmen Sie einer Veröffentlichung folgender öffentlicher Einkaufsdaten zu?

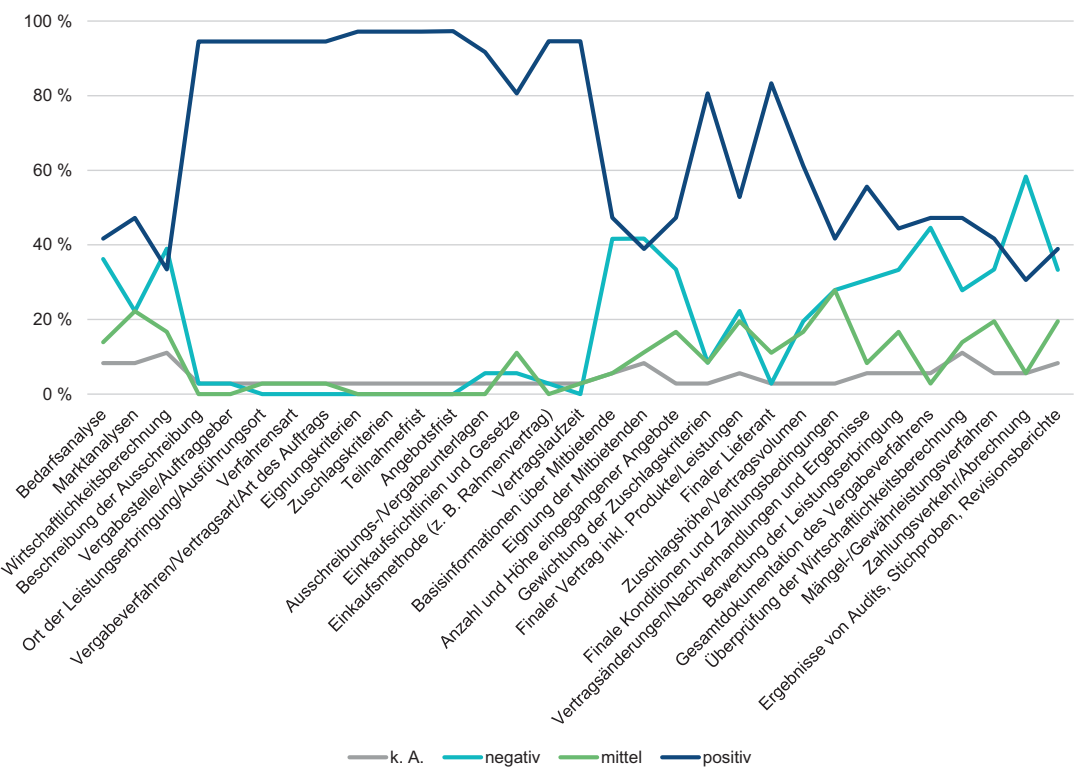

Abbildung 8.14 Künftige Veröffentlichung öffentlicher Einkaufsdaten

Tabelle 8.26 Künftige Veröffentlichung öffentlicher Einkaufsdaten

\begin{tabular}{l|c|c|c|c|c|c|c|c}
\hline \multirow{2}{*}{ Antworten } & \multicolumn{2}{|c|}{ Positiv } & \multicolumn{2}{c|}{ Mittel } & \multicolumn{2}{c|}{ Negativ } & \multicolumn{2}{c}{ k. A. } \\
\cline { 2 - 10 } & $\boldsymbol{\%}$ & Anz. & $\%$ & Anz. & $\%$ & Anz. & $\%$ & Anz. \\
\hline Planung & & & & & & & & \\
\hline Bedarfsanalyse & $42 \%$ & 15 & $14 \%$ & 5 & $36 \%$ & 13 & $8 \%$ & 3 \\
\hline Marktanalysen & $47 \%$ & 17 & $22 \%$ & 8 & $22 \%$ & 8 & $8 \%$ & 3 \\
\hline $\begin{array}{l}\text { Wirtschaftlichkeits- } \\
\text { berechnung }\end{array}$ & $33 \%$ & 12 & $17 \%$ & 6 & $39 \%$ & 14 & $11 \%$ & 4 \\
\hline Mittelwert & $\mathbf{4 1} \%$ &. & $\mathbf{1 8 \%}$ &. & $\mathbf{3 2} \%$ &. & $\mathbf{9 \%}$ &. \\
\hline
\end{tabular}

(Fortsetzung) 
Tabelle 8.26 (Fortsetzung)

\begin{tabular}{|c|c|c|c|c|c|c|c|c|}
\hline \multirow{2}{*}{ Antworten } & \multicolumn{2}{|c|}{ Positiv } & \multicolumn{2}{|c|}{ Mittel } & \multicolumn{2}{|c|}{ Negativ } & \multicolumn{2}{|c|}{ k. A. } \\
\hline & $\%$ & Anz. & $\%$ & Anz. & $\%$ & Anz. & $\%$ & Anz. \\
\hline \multicolumn{9}{|l|}{ Ausschreibung } \\
\hline $\begin{array}{l}\text { Beschreibung der } \\
\text { Ausschreibung }\end{array}$ & $95 \%$ & 34 & $0 \%$ & 0 & $3 \%$ & 1 & $3 \%$ & 1 \\
\hline $\begin{array}{l}\text { Vergabestelle/ } \\
\text { Auftraggeber }\end{array}$ & $95 \%$ & 34 & $0 \%$ & 0 & $3 \%$ & 1 & $3 \%$ & 1 \\
\hline $\begin{array}{l}\text { Ort der Leistungser- } \\
\text { bringung }\end{array}$ & $95 \%$ & 34 & $3 \%$ & 1 & $0 \%$ & 0 & $3 \%$ & 1 \\
\hline Verfahrensart & $95 \%$ & 34 & $3 \%$ & 1 & $0 \%$ & 0 & $3 \%$ & 1 \\
\hline $\begin{array}{l}\text { Vergabeverfahren/ } \\
\text { Vertragsart }\end{array}$ & $95 \%$ & 34 & $3 \%$ & 1 & $0 \%$ & 0 & $3 \%$ & 1 \\
\hline Eignungskriterien & $97 \%$ & 35 & $0 \%$ & 0 & $0 \%$ & 0 & $3 \%$ & 1 \\
\hline Zuschlagskriterien & $97 \%$ & 35 & $0 \%$ & 0 & $0 \%$ & 0 & $3 \%$ & 1 \\
\hline Teilnahmefrist & $97 \%$ & 35 & $0 \%$ & 0 & $0 \%$ & 0 & $3 \%$ & 1 \\
\hline Angebotsfrist & $97 \%$ & 35 & $0 \%$ & 0 & $0 \%$ & 0 & $3 \%$ & 1 \\
\hline $\begin{array}{l}\text { Ausschreibungsunter- } \\
\text { lagen }\end{array}$ & $92 \%$ & 33 & $0 \%$ & 0 & $6 \%$ & 2 & $3 \%$ & 1 \\
\hline $\begin{array}{l}\text { Einkaufsrichtlinien } \\
\text { und Gesetze }\end{array}$ & $81 \%$ & 29 & $11 \%$ & 4 & $6 \%$ & 2 & $3 \%$ & 1 \\
\hline Einkaufsmethode & $95 \%$ & 34 & $0 \%$ & 0 & $3 \%$ & 1 & $3 \%$ & 1 \\
\hline Vertragslaufzeit & $95 \%$ & 34 & $3 \%$ & 1 & $0 \%$ & 0 & $3 \%$ & 1 \\
\hline $\begin{array}{l}\text { Basisinformationen } \\
\text { Mitbietende }\end{array}$ & $47 \%$ & 17 & $6 \%$ & 2 & $42 \%$ & 15 & $6 \%$ & 2 \\
\hline Mittelwert & $91 \%$ &. & $2 \%$ &.$\%$ & $4 \%$ &. & $3 \%$ &. \\
\hline \multicolumn{9}{|l|}{ Bewertung } \\
\hline $\begin{array}{l}\text { Eignung der } \\
\text { Mitbietenden }\end{array}$ & $39 \%$ & 14 & $11 \%$ & 4 & $42 \%$ & 15 & $8 \%$ & 3 \\
\hline $\begin{array}{l}\text { Anzahl/Höhe eingeg. } \\
\text { Angebote }\end{array}$ & $47 \%$ & 17 & $17 \%$ & 6 & $33 \%$ & 12 & $3 \%$ & 1 \\
\hline $\begin{array}{l}\text { Gewichtung der } \\
\text { Zuschlagskriterien }\end{array}$ & $81 \%$ & 29 & $8 \%$ & 3 & $8 \%$ & 3 & $3 \%$ & 1 \\
\hline Mittelwert & $56 \%$ &. & $12 \%$ &. & $28 \%$ &. & $5 \%$ &. \\
\hline
\end{tabular}

(Fortsetzung) 
Tabelle 8.26 (Fortsetzung)

\begin{tabular}{|c|c|c|c|c|c|c|c|c|}
\hline \multirow[t]{2}{*}{ Antworten } & \multicolumn{2}{|c|}{ Positiv } & \multicolumn{2}{|c|}{ Mittel } & \multicolumn{2}{|c|}{ Negativ } & \multicolumn{2}{|c|}{ k. A. } \\
\hline & $\%$ & Anz. & $\%$ & Anz. & $\%$ & Anz. & $\%$ & Anz. \\
\hline \multicolumn{9}{|l|}{ Vergabe } \\
\hline Finaler Vertrag & $53 \%$ & 19 & $20 \%$ & 7 & $22 \%$ & 8 & $6 \%$ & 2 \\
\hline Finaler Lieferant & $83 \%$ & 30 & $11 \%$ & 4 & $3 \%$ & 1 & $3 \%$ & 1 \\
\hline $\begin{array}{l}\text { Zuschlagshöhe/ } \\
\text { Vertragsvolumen }\end{array}$ & $61 \%$ & 22 & $17 \%$ & 6 & $20 \%$ & 7 & $3 \%$ & 1 \\
\hline $\begin{array}{l}\text { Finale Konditionen/ } \\
\text { Zahlungsbed. }\end{array}$ & $42 \%$ & 15 & $28 \%$ & 10 & $28 \%$ & 10 & $3 \%$ & 1 \\
\hline Mittelwert & $60 \%$ &. & $19 \%$ & $\%$ & $18 \%$ &. & $4 \%$ &. \\
\hline \multicolumn{9}{|l|}{ Ausführung } \\
\hline $\begin{array}{l}\text { Vertragsänderungen \& } \\
\text { Ergebnisse }\end{array}$ & $56 \%$ & 20 & $8 \%$ & 3 & $31 \%$ & 11 & $6 \%$ & 2 \\
\hline $\begin{array}{l}\text { Bewertung Leistungser- } \\
\text { bringung }\end{array}$ & $44 \%$ & 16 & $17 \%$ & 6 & $33 \%$ & 12 & $6 \%$ & 2 \\
\hline Gesamtdokumentation & $47 \%$ & 17 & $3 \%$ & 1 & $45 \%$ & 16 & $6 \%$ & 2 \\
\hline $\begin{array}{l}\text { Überprüfung Business } \\
\text { Case }\end{array}$ & $47 \%$ & 17 & $14 \%$ & 5 & $28 \%$ & 10 & $11 \%$ & 4 \\
\hline $\begin{array}{l}\text { Mängel-/Gewähr- } \\
\text { leistungsverfahren }\end{array}$ & $42 \%$ & 15 & $20 \%$ & 7 & $33 \%$ & 12 & $6 \%$ & 2 \\
\hline $\begin{array}{l}\text { Zahlungsverkehr/ } \\
\text { Abrechnung }\end{array}$ & $31 \%$ & 11 & $6 \%$ & 2 & $58 \%$ & 21 & $6 \%$ & 2 \\
\hline $\begin{array}{l}\text { Ergebnisse von Audits, } \\
\text { Stichproben }\end{array}$ & $39 \%$ & 14 & $20 \%$ & 7 & $33 \%$ & 12 & $8 \%$ & 3 \\
\hline Mittelwert & $44 \%$ &.$\%$ & $12 \%$ &.$\%$ & $37 \%$ &.$\%$ & $7 \%$ &.$\%$ \\
\hline Summe &. & 757 &. & 100 &. & 207 &.$\%$ & 52 \\
\hline Mittelwert & $68 \%$ & 24 & $9 \%$ & 3 & $19 \%$ & 7 & $5 \%$ & 2 \\
\hline
\end{tabular}

\section{Bewertung:}

Auf die Frage, inwieweit welche öffentlichen Einkaufsdaten künftig veröffentlicht werden sollten, befürworten im Mittel $41 \%$ für den Teilprozess der Planung eine solche Veröffentlichung. Mit $91 \%$ erfährt allerdings der Teilprozess der Ausschreibung die höchste Zustimmung. Die Teilprozesse Bewertung und Vergabe liegen mit im Mittel $56 \%$ respektive $60 \%$ auf ähnlichem Zustimmungsniveau. Etwas geringer fällt die Zustimmung mit $44 \%$ für den Teilprozess der Aus- 
führung aus - hier ist gleichzeitig der Wert der Ablehnung mit $37 \%$ vergleichbar hoch. Die Daten sind in Abbildung 8.14/Tabelle 8.26 dargestellt. Etwas weiter hinten, in Abbildung 8.34, findet sich der Vergleich zwischen heutiger und künftiger Veröffentlichung öffentlicher Einkaufsdaten, auf den in Abschnitt 8.2.4 noch näher eingegangen wird.

Frage 14: Wer sollte aus Ihrer Sicht zukünftig Zugriff auf welche veröffentlichten öffentlichen Einkaufsdaten haben?

$100 \%$

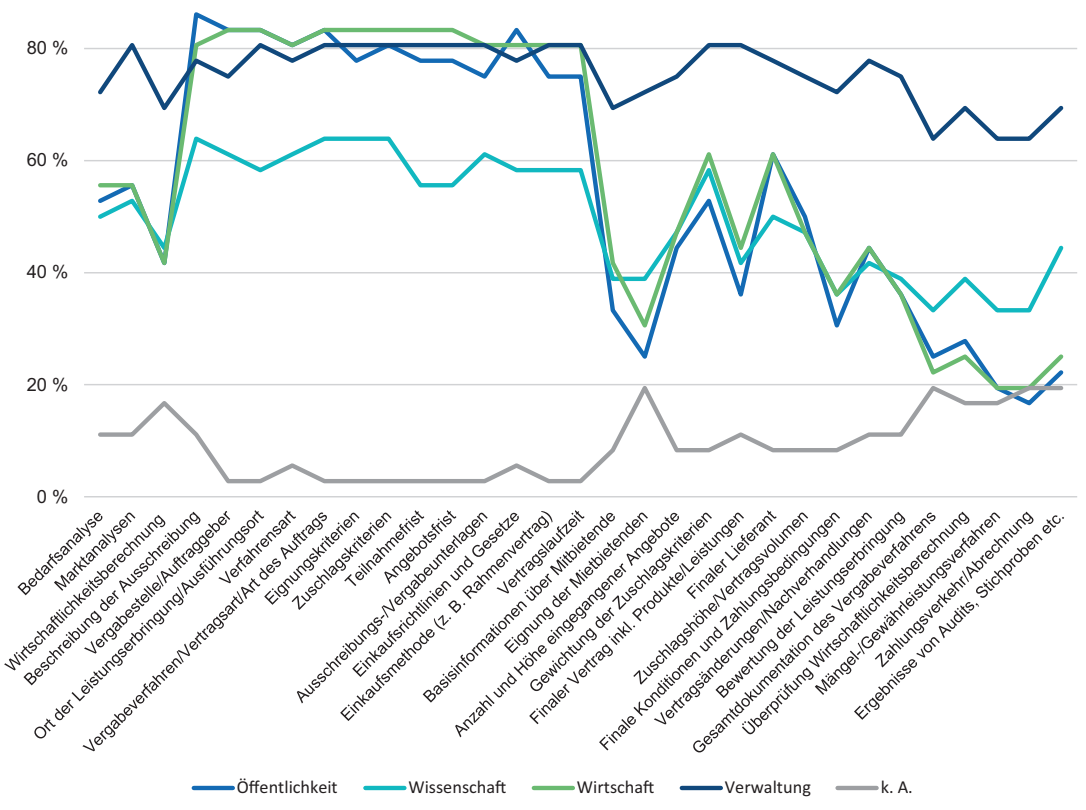

Abbildung 8.15 Künftiger Zugriff einzelner Gruppen auf öffentliche Einkaufsdaten 
Tabelle 8.27 Künftiger Zugriff einzelner Gruppen auf öffentliche Einkaufsdaten

\begin{tabular}{|c|c|c|c|c|c|c|c|c|c|c|}
\hline \multirow{2}{*}{ Antworten } & \multicolumn{2}{|c|}{ Öffentl. } & \multicolumn{2}{|c|}{ Wiss. } & \multicolumn{2}{|c|}{ Wirtschaft } & \multicolumn{2}{|c|}{ Verw. } & \multicolumn{2}{|c|}{ k. A. } \\
\hline & $\%$ & Anz. & $\%$ & Anz. & $\%$ & Anz. & $\%$ & Anz. & $\%$ & Anz. \\
\hline \multicolumn{11}{|l|}{ Planung } \\
\hline Bedarfsanalyse & $53 \%$ & 19 & $50 \%$ & 18 & $56 \%$ & 20 & $72 \%$ & 26 & $11 \%$ & 4 \\
\hline Marktanalysen & $56 \%$ & 20 & $53 \%$ & 19 & $56 \%$ & 20 & $81 \%$ & 29 & $11 \%$ & 4 \\
\hline $\begin{array}{l}\text { Wirtschaftlichkeits- } \\
\text { berechnung }\end{array}$ & $42 \%$ & 15 & $44 \%$ & 16 & $42 \%$ & 15 & $69 \%$ & 25 & $17 \%$ & 6 \\
\hline Mittelwert & $50 \%$ & $\%$ & $49 \%$ &.$\%$ & $51 \%$ &.$\%$ & $74 \%$ &.$\%$ & $13 \%$ & $\%$ \\
\hline \multicolumn{11}{|l|}{ Ausschreibung } \\
\hline $\begin{array}{l}\text { Beschreibung der } \\
\text { Ausschreibung }\end{array}$ & $86 \%$ & 31 & $64 \%$ & 23 & $81 \%$ & 29 & $78 \%$ & 28 & $11 \%$ & 4 \\
\hline $\begin{array}{l}\text { Vergabestelle/Auf- } \\
\text { traggeber }\end{array}$ & $83 \%$ & 30 & $61 \%$ & 22 & $83 \%$ & 30 & $75 \%$ & 27 & $3 \%$ & 1 \\
\hline $\begin{array}{l}\text { Ort der Leistungser- } \\
\text { bringung }\end{array}$ & $83 \%$ & 30 & $58 \%$ & 21 & $83 \%$ & 30 & $81 \%$ & 29 & $3 \%$ & 1 \\
\hline Verfahrensart & $81 \%$ & 29 & $61 \%$ & 22 & $81 \%$ & 29 & $78 \%$ & 28 & $6 \%$ & 2 \\
\hline $\begin{array}{l}\text { Vergabeverfahren/ } \\
\text { Vertragsart }\end{array}$ & $83 \%$ & 30 & $64 \%$ & 23 & $83 \%$ & 30 & $81 \%$ & 29 & $3 \%$ & 1 \\
\hline Eignungskriterien & $78 \%$ & 28 & $64 \%$ & 23 & $83 \%$ & 30 & $81 \%$ & 29 & $3 \%$ & 1 \\
\hline Zuschlagskriterien & $81 \%$ & 29 & $64 \%$ & 23 & $83 \%$ & 30 & $81 \%$ & 29 & $3 \%$ & 1 \\
\hline Teilnahmefrist & $78 \%$ & 28 & $56 \%$ & 20 & $83 \%$ & 30 & $81 \%$ & 29 & $3 \%$ & 1 \\
\hline Angebotsfrist & $78 \%$ & 28 & $56 \%$ & 20 & $83 \%$ & 30 & $81 \%$ & 29 & $3 \%$ & 1 \\
\hline $\begin{array}{l}\text { Ausschreibungs- } \\
\text { unterlagen }\end{array}$ & $75 \%$ & 27 & $61 \%$ & 22 & $81 \%$ & 29 & $81 \%$ & 29 & $3 \%$ & 1 \\
\hline $\begin{array}{l}\text { Einkaufsrichtlinien } \\
\text { und Gesetze }\end{array}$ & $83 \%$ & 30 & $58 \%$ & 21 & $81 \%$ & 29 & $78 \%$ & 28 & $6 \%$ & 2 \\
\hline Einkaufsmethode & $75 \%$ & 27 & $58 \%$ & 21 & $81 \%$ & 29 & $81 \%$ & 29 & $3 \%$ & 1 \\
\hline Vertragslaufzeit & $75 \%$ & 27 & $58 \%$ & 21 & $81 \%$ & 29 & $81 \%$ & 29 & $3 \%$ & 1 \\
\hline $\begin{array}{l}\text { Basisinformationen } \\
\text { Mitbietende }\end{array}$ & $33 \%$ & 12 & $39 \%$ & 14 & $42 \%$ & 15 & $69 \%$ & 25 & $8 \%$ & 3 \\
\hline Mittelwert & $77 \%$ &. & $59 \%$ & $\%$ & $79 \%$ &. & $79 \%$ &. & $4 \%$ &. \\
\hline \multicolumn{11}{|l|}{ Bewertung } \\
\hline $\begin{array}{l}\text { Eignung der Mit- } \\
\text { bietenden }\end{array}$ & $25 \%$ & 9 & $39 \%$ & 14 & $31 \%$ & 11 & $72 \%$ & 26 & $19 \%$ & 7 \\
\hline
\end{tabular}


Tabelle 8.27 (Fortsetzung)

\begin{tabular}{|c|c|c|c|c|c|c|c|c|c|c|}
\hline \multirow{2}{*}{ Antworten } & \multicolumn{2}{|c|}{ Öffentl. } & \multicolumn{2}{|c|}{ Wiss. } & \multicolumn{2}{|c|}{ Wirtschaft } & \multicolumn{2}{|c|}{ Verw. } & \multicolumn{2}{|c|}{ k. A. } \\
\hline & $\%$ & Anz. & $\%$ & Anz. & $\%$ & Anz. & $\%$ & Anz. & $\%$ & Anz. \\
\hline $\begin{array}{l}\text { Anzahl/Höhe } \\
\text { eingeg. Angebote }\end{array}$ & $44 \%$ & 16 & $47 \%$ & 17 & $47 \%$ & 17 & $75 \%$ & 27 & $8 \%$ & 3 \\
\hline $\begin{array}{l}\text { Gewichtung } \\
\text { Zuschlagskriterien }\end{array}$ & $53 \%$ & 19 & $58 \%$ & 21 & $61 \%$ & 22 & $81 \%$ & 29 & $8 \%$ & 3 \\
\hline Mittelwert & $41 \%$ &. & $48 \%$ &. & $46 \%$ &. & $76 \%$ &. & $12 \%$ &. \\
\hline \multicolumn{11}{|l|}{ Vergabe } \\
\hline Finaler Vertrag & $36 \%$ & 13 & $42 \%$ & 15 & $44 \%$ & 16 & $81 \%$ & 29 & $11 \%$ & 4 \\
\hline Finaler Lieferant & $61 \%$ & 22 & $50 \%$ & 18 & $61 \%$ & 22 & $78 \%$ & 28 & $8 \%$ & 3 \\
\hline $\begin{array}{l}\text { Zuschlagshöhe/Ver- } \\
\text { tragsvolumen }\end{array}$ & $50 \%$ & 18 & $47 \%$ & 17 & $47 \%$ & 17 & $75 \%$ & 27 & $8 \%$ & 3 \\
\hline $\begin{array}{l}\text { Finale Konditionen/ } \\
\text { Zahlungsbed. }\end{array}$ & $31 \%$ & 11 & $36 \%$ & 13 & $36 \%$ & 13 & $72 \%$ & 26 & $8 \%$ & 3 \\
\hline Mittelwert & $44 \%$ &. & $44 \%$ &. & $47 \%$ &. & $76 \%$ &. & $9 \%$ & $\%$ \\
\hline \multicolumn{11}{|l|}{ Ausführung } \\
\hline Vertragsänderungen & $44 \%$ & 16 & $42 \%$ & 15 & $44 \%$ & 16 & $78 \%$ & 28 & $11 \%$ & 4 \\
\hline $\begin{array}{l}\text { Bewertung } \\
\text { Leistungserbringung }\end{array}$ & $36 \%$ & 13 & $39 \%$ & 14 & $36 \%$ & 13 & $75 \%$ & 27 & $11 \%$ & 4 \\
\hline $\begin{array}{l}\text { Gesamt- } \\
\text { dokumentation }\end{array}$ & $25 \%$ & 9 & $33 \%$ & 12 & $22 \%$ & 8 & $64 \%$ & 23 & $19 \%$ & 7 \\
\hline $\begin{array}{l}\text { Überprüfung } \\
\text { Business Case }\end{array}$ & $28 \%$ & 10 & $39 \%$ & 14 & $25 \%$ & 9 & $69 \%$ & 25 & $17 \%$ & 6 \\
\hline $\begin{array}{l}\text { Mängel-/ } \\
\text { Gewährleistungsverf. }\end{array}$ & $19 \%$ & 7 & $33 \%$ & 12 & $19 \%$ & 7 & $64 \%$ & 23 & $17 \%$ & 6 \\
\hline $\begin{array}{l}\text { Zahlungsverkehr/ } \\
\text { Abrechnung }\end{array}$ & $17 \%$ & 6 & $33 \%$ & 12 & $19 \%$ & 7 & $64 \%$ & 23 & $19 \%$ & 7 \\
\hline $\begin{array}{l}\text { Ergebnisse Audits, } \\
\text { Stichproben }\end{array}$ & $22 \%$ & 8 & $44 \%$ & 16 & $25 \%$ & 9 & $69 \%$ & 25 & $19 \%$ & 7 \\
\hline Mittelwert & $27 \%$ &. & $38 \%$ &. & $27 \%$ &.$\%$ & $69 \%$ &.$\%$ & $16 \%$ & $\%$ \\
\hline Summe &. & 617 & $\%$ & 559 &. & 641 &. & 843 &. & 102 \\
\hline Mittelwert & $55 \%$ & 20 & $50 \%$ & 18 & $56 \%$ & 20 & $72 \%$ & 26 & $9 \%$ & 3 \\
\hline
\end{tabular}




\section{Bewertung:}

Auf die Frage (Mehrfachauswahl), wer künftig Zugriff auf welche öffentlichen Einkaufsdaten haben sollte, spricht sich gemäß Abbildung 8.15/Tabelle 8.27 eine deutliche Mehrheit für die Verwaltung aus (Spannweite zwischen im Mittel $69 \%$ bis $74 \%$ der Zustimmung je nach Teilprozess; über alle Teilprozesse im Mittel $72 \%$ Zustimmung).

Die Wirtschaft ist die Gruppe mit der zweithöchsten Zustimmung mit einem Mittelwert von $56 \%$ über alle Teilprozesse. Die Spannweite rangiert von im Mittel $27 \%$ im Teilprozess der Ausführung bis $79 \%$ im Teilprozess der Ausschreibung. Das heißt, die Zustimmung variiert hier stärker als bei der Verwaltung. Nach wie vor ist die Zustimmung der Befragten für einen Datenzugriff durch die Wirtschaft in der Phase der Ausschreibung am Höchsten. Es folgen abnehmend die Zustimmungen zum Zugriff auf Daten der Teilprozesse Vergabe, Planung, Bewertung und Ausführung.

An dritter Stelle und nur kurz dahinter steht die Öffentlichkeit mit einem Mittelwert über alle Teilprozesse von $55 \%$ (Spannweite von im Mittel $27 \%$ im Teilprozess der Ausführung bis $77 \%$ im Mittel im Teilprozess der Ausschreibung). Das Verlaufsmuster von Wirtschaft und Öffentlichkeit ist über den Gesamtprozess hinweg identisch. Lediglich bei den Teilprozessen der Bewertung und Vergabe gibt es im Vergleich Abweichungen: Im Teilprozess der Bewertung liegt die Wirtschaft bei einer Zustimmung von $46 \%$, die Öffentlichkeit bei $41 \%$. Bei der Vergabe weist die Wirtschaft ebenfalls eine leicht höhere Zustimmung von $47 \%$ gegenüber der Öffentlichkeit von $44 \%$ auf.

An letzter Stelle steht die Wissenschaft mit im Mittel $50 \%$ (Spannweite von im Mittel $38 \%$ im Teilprozess der Ausführung bis 59\% im Mittel im Teilprozess der Ausschreibung). Hier ist die Streuung wieder geringer, allerdings offenbart der Blick in die Teilprozesse ein interessantes Muster: Man möchte demnach der Wissenschaft einen stärkeren Einblick in den Teilprozess der Bewertung gewähren. Mit im Mittel $48 \%$ liegt dieser Wert vor der Wirtschaft mit $46 \%$ und vor der Öffentlichkeit mit $41 \%$. Noch deutlicher fällt dies für den Teilprozess der Ausführung aus. Hier liegt die Wissenschaft mit $38 \%$ Zustimmung vor der Wirtschaft und Öffentlichkeit mit nur jeweils $27 \%$. Mit Blick auf den Teilprozess der Ausschreibung sieht man die Wissenschaft allerdings abgeschlagen an letzter Stelle mit nur $59 \%$ Zustimmung gegenüber der Wirtschaft mit $79 \%$ und der Öffentlichkeit mit $77 \%$. 
Frage 15: Für welche Art der Projekte sollte die Öffnung der öffentlichen Einkaufsdaten mehr oder weniger ermöglicht beziehungsweise eingeschränkt werden?

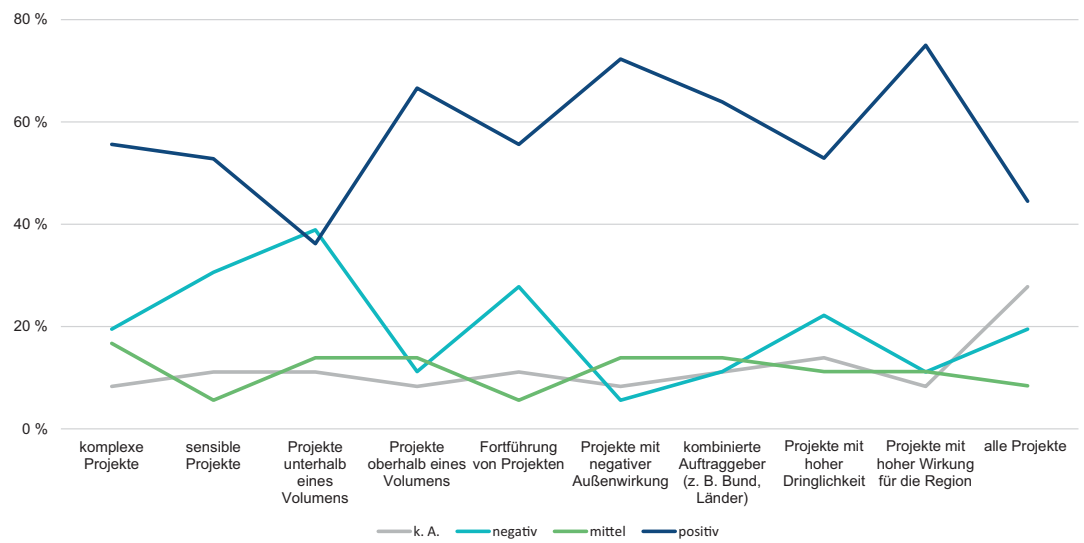

Abbildung 8.16 Einschränkung der Öffnung der öffentlichen Einkaufsdaten (Projekte)

Tabelle 8.28 Einschränkung der Öffnung der öffentlichen Einkaufsdaten (Projekte)

\begin{tabular}{l|c|c|c|c|c|c|c|c}
\hline \multirow{2}{*}{ Antworten } & \multicolumn{2}{|c|}{ Positiv } & \multicolumn{2}{c|}{ Mittel } & \multicolumn{2}{c|}{ Negativ } & \multicolumn{2}{c}{ k. A. } \\
\cline { 2 - 10 } & $\%$ & Anz. & $\%$ & Anz. & $\%$ & Anz. & $\%$ & Anz. \\
\hline komplexe Projekte & $56 \%$ & 20 & $17 \%$ & 6 & $20 \%$ & 7 & $8 \%$ & 3 \\
\hline sensible Projekte & $53 \%$ & 19 & $6 \%$ & 2 & $31 \%$ & 11 & $11 \%$ & 4 \\
\hline $\begin{array}{l}\text { Projekte unterhalb } \\
\text { eines Volumens }\end{array}$ & $36 \%$ & 13 & $14 \%$ & 5 & $39 \%$ & 14 & $11 \%$ & 4 \\
\hline $\begin{array}{l}\text { Projekte oberhalb } \\
\text { eines Volumens }\end{array}$ & $67 \%$ & 24 & $14 \%$ & 5 & $11 \%$ & 4 & $8 \%$ & 3 \\
\hline $\begin{array}{l}\text { Fortführung von } \\
\text { Projekten }\end{array}$ & $56 \%$ & 20 & $6 \%$ & 2 & $28 \%$ & 10 & $11 \%$ & 4 \\
\hline $\begin{array}{l}\text { Projekte mit negativer } \\
\text { Außenwirkung }\end{array}$ & $72 \%$ & 26 & $14 \%$ & 5 & $6 \%$ & 2 & $8 \%$ & 3 \\
\hline
\end{tabular}

(Fortsetzung) 
Tabelle $\mathbf{8 . 2 8}$ (Fortsetzung)

\begin{tabular}{l|c|c|c|c|c|c|c|c}
\hline \multirow{2}{*}{ Antworten } & \multicolumn{2}{|c|}{ Positiv } & \multicolumn{2}{c|}{ Mittel } & \multicolumn{2}{c|}{ Negativ } & \multicolumn{2}{c}{ k. A. } \\
\cline { 2 - 10 } & $\boldsymbol{\%}$ & $\mathbf{A n z}$ & $\boldsymbol{\%}$ & $\mathbf{A n z}$ & $\boldsymbol{\%}$ & $\mathbf{A n z}$ & $\%$ & Anz. \\
\hline $\begin{array}{l}\text { kombinierte Auftrag- } \\
\text { geber }\end{array}$ & $64 \%$ & 23 & $14 \%$ & 5 & $11 \%$ & 4 & $11 \%$ & 4 \\
\hline $\begin{array}{l}\text { Projekte mit hoher } \\
\text { Dringlichkeit }\end{array}$ & $53 \%$ & 19 & $11 \%$ & 4 & $22 \%$ & 8 & $14 \%$ & 5 \\
\hline $\begin{array}{l}\text { Projekte mit hoher } \\
\text { regionaler Wirkung }\end{array}$ & $75 \%$ & 27 & $11 \%$ & 4 & $11 \%$ & 4 & $8 \%$ & 3 \\
\hline $\begin{array}{l}\text { alle Projekte } \\
\text { Summe }\end{array}$ & $45 \%$ & 16 & $8 \%$ & 3 & $20 \%$ & 7 & $28 \%$ & 10 \\
\hline Mittelwert & $\mathbf{5 8} \%$ & $\mathbf{2 1}$ & $\mathbf{1 1} \%$ & $\mathbf{4}$ & $\mathbf{2 0} \%$ & $\mathbf{7}$ & $\mathbf{1 2} \%$ & $\mathbf{4}$ \\
\hline
\end{tabular}

\section{Bewertung:}

Auf die Frage, für welche Art der Projekte die Öffnung der öffentlichen Einkaufsdaten mehr oder weniger ermöglicht beziehungsweise eingeschränkt werden sollte, lassen sich die Antworten wie folgt zusammenfassen (siehe Abbildung 8.16/Tabelle 8.28): Eine deutliche Zustimmung im Sinne einer stärkeren Öffnung der öffentlichen Einkaufsdaten erhalten Projekte mit hoher Wirkung für die Region (75\%), Projekte mit negativer Außenwirkung (72\%), Projekte mit kombinierten Auftraggebern (64\%) sowie Projekte oberhalb eines bestimmten Volumens in Euro (67\%).

Unterhalb des Mittelwerts, aber immer noch in der Kategorie „positiv“, rangieren „alle Projekte“ (45\%) sowie Projekte unterhalb eines bestimmten Volumens in Euro (36\%). Gleichzeitig sehen $39 \%$ der Befragten in der Kategorie „negativ“ keine Möglichkeit der Eindämmung von Korruption durch die Öffnung öffentlicher Einkaufsdaten für Projekte unterhalb eines bestimmten Volumens.

Dies mag ein Hinweis darauf sein, dass anhand bestimmter Kriterien eine bestimmte Wertgrenze diskutiert und festgelegt werden müsste, unterhalb derer keine Veröffentlichung öffentlicher Einkaufsdaten erfolgt. Gleichzeitig zeigt dies den Differenzierungsbedarf, aber auch -ansätze auf. 
Frage 16: Gibt es aus Ihrer Sicht Warengruppen oder Sektoren, deren Daten nicht veröffentlicht werden sollten? Wenn ja, welche und warum?

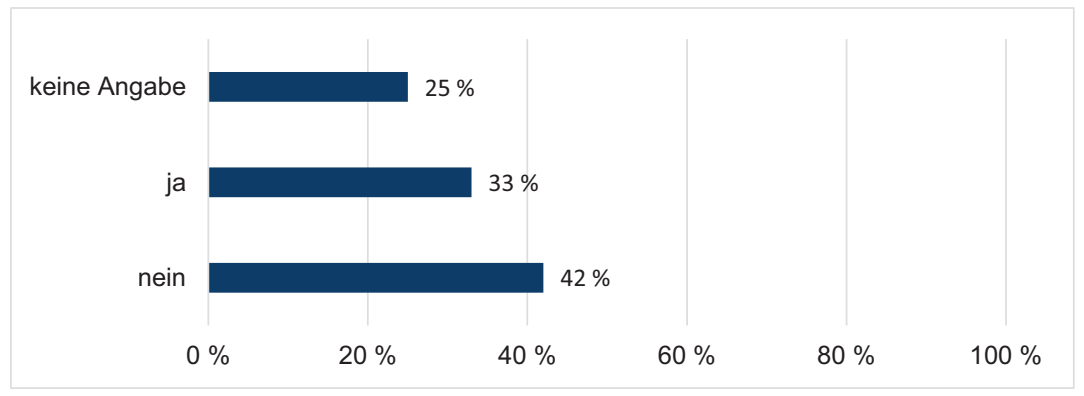

Abbildung 8.17 Einschränkungen der Öffnung öffentlicher Einkaufsdaten (Warengruppen)

Tabelle 8.29 Einschränkungen der Öffnung öffentlicher Einkaufsdaten (Warengruppen)

\begin{tabular}{l|c|c}
\hline Antworten & Prozent & Anzahl \\
\hline nein & $42 \%$ & 15 \\
\hline ja & $33 \%$ & 12 \\
\hline keine Angabe & $25 \%$ & 9 \\
\hline Summe &.$\%$ & $\mathbf{3 6}$ \\
\hline
\end{tabular}

\section{Bewertung:}

Auf die Frage, ob es Warengruppen oder Sektoren gibt, deren Daten nicht veröffentlicht werden sollten, antworten $42 \%$ der Teilnehmer gemäß Abbildung 8.17/Tabelle 8.29 mit ,nein“, $33 \%$ mit ,ja“. Hier werden erläuternd insbesondere die Waren und der Sektor der Landesverteidigung genannt. Bei dieser Frage ist der Anteil von $25 \%$ mit ,keine Angabe“ recht hoch, was auf eine gewisse Unentschlossenheit verweist.

So zeigen die Angaben unter ,sonstige“ im Freitext weiteren Austauschbedarf auf. Einige Teilnehmer votieren dafür, sicherheitsrelevante Waren beziehungsweise Sektoren, Themen rund um die Polizei sowie (teilweise) Themen der (Landes-)Verteidigung von der Veröffentlichung auszunehmen. Andere Teilnehmer stimmen insoweit zu, als ein schrittweises Vorgehen, zum Beispiel bei Verteidigung und Sicherheit, sinnvoll wäre, weisen aber auch darauf hin, dass 
in den meisten Fällen eine Veröffentlichung über einen bestimmten Zeitraum möglich sein sollte. Dies unterstreicht auch die OCP (Open Contracting Partnership, 2019e, S. 34 ff.). Demnach zeichnet sich in diesem Themenfeld ebenfalls Abstimmungsbedarf dazu ab, inwieweit bestimmte Bereiche in punkto Öffnung öffentlicher Einkaufsdaten sensibler angegangen werden müssten.

\section{Frage 17: Wo sollten die von Ihnen benannten öffentlichen Einkaufsdaten künftig veröffentlicht werden?}

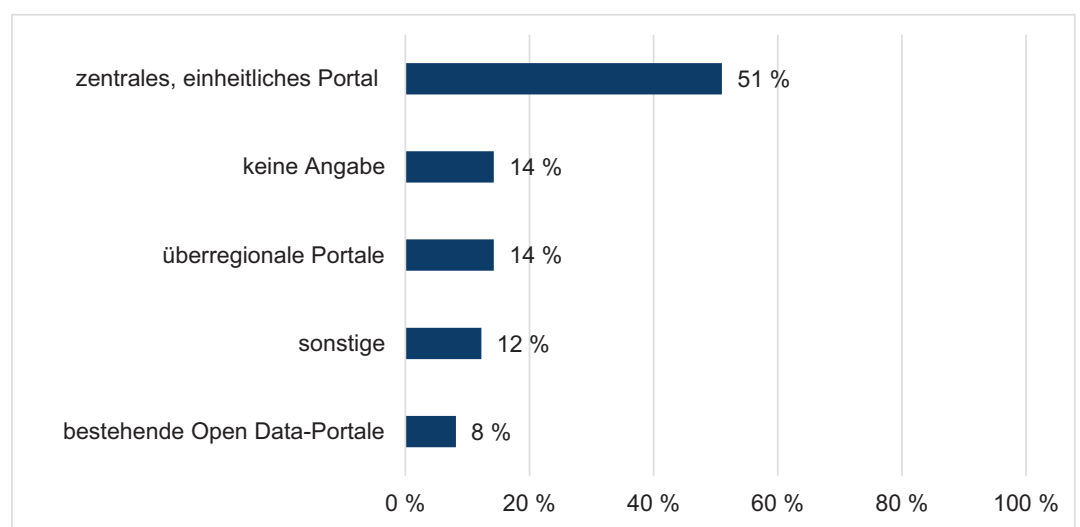

Abbildung 8.18 Künftige Bereitstellungsorte öffentlicher Einkaufsdaten

Tabelle 8.30 Künftige Bereitstellungsorte nach Antworthäufigkeit

\begin{tabular}{l|c|c}
\hline Antworten & Prozent & Anzahl \\
\hline zentrales, einheitliches Portal & $51 \%$ & 25 \\
\hline keine Angabe & $14 \%$ & 7 \\
\hline überregionale Portale & $14 \%$ & 7 \\
\hline sonstige & $12 \%$ & 6 \\
\hline bestehende Open Data-Portale & $8 \%$ & 4 \\
\hline Summe &. & $\mathbf{4 9}$ \\
\hline
\end{tabular}

\section{Bewertung:}

Auf die Frage (offene Nennungen), wo die benannten öffentlichen Einkaufsdaten künftig veröffentlicht werden sollten, wurden die Nennungen in Themenbereiche geclustert (siehe Abbildung 8.18/Tabelle 8.30). 
$51 \%$ sprechen sich für ein zentrales, einheitliches Portal aus. Hierunter fallen https://www.service.bund.de (acht Nennungen), https://www.govdata.de (vier Nennungen) und https://ted.europa.eu (drei Nennungen) oder allgemeine Nennungen wie ,ein einheitliches/zentrales Portal“.

$14 \%$ der Teilnehmer befürworten überregionale Portale wie Vergabemarktplätze, Bundesportale, Vergabeportale von Bund und Ländern oder Landesportale. Nur $8 \%$ der Befragten unterstützen die Integration in bestehende Open Data-Portale. „Keine Angabe“ machten $14 \%$ der Befragten. Unter "sonstige“ fallen zum Beispiel Nennungen für das Ratsinformationssystem, Statistikämter, den Vergabemarktplatz Rheinland und den Bundesanzeiger.

Insgesamt zeichnet sich deutlich die Befürwortung eines zentralen Portals für die Offenlegung öffentlicher Einkaufsdaten ab.

\section{Frage 18: Welche wirtschaftlichen Konsequenzen hätte die Bereitstellung offener öffentlicher Einkaufsdaten über den govdata.de-Verbund?}

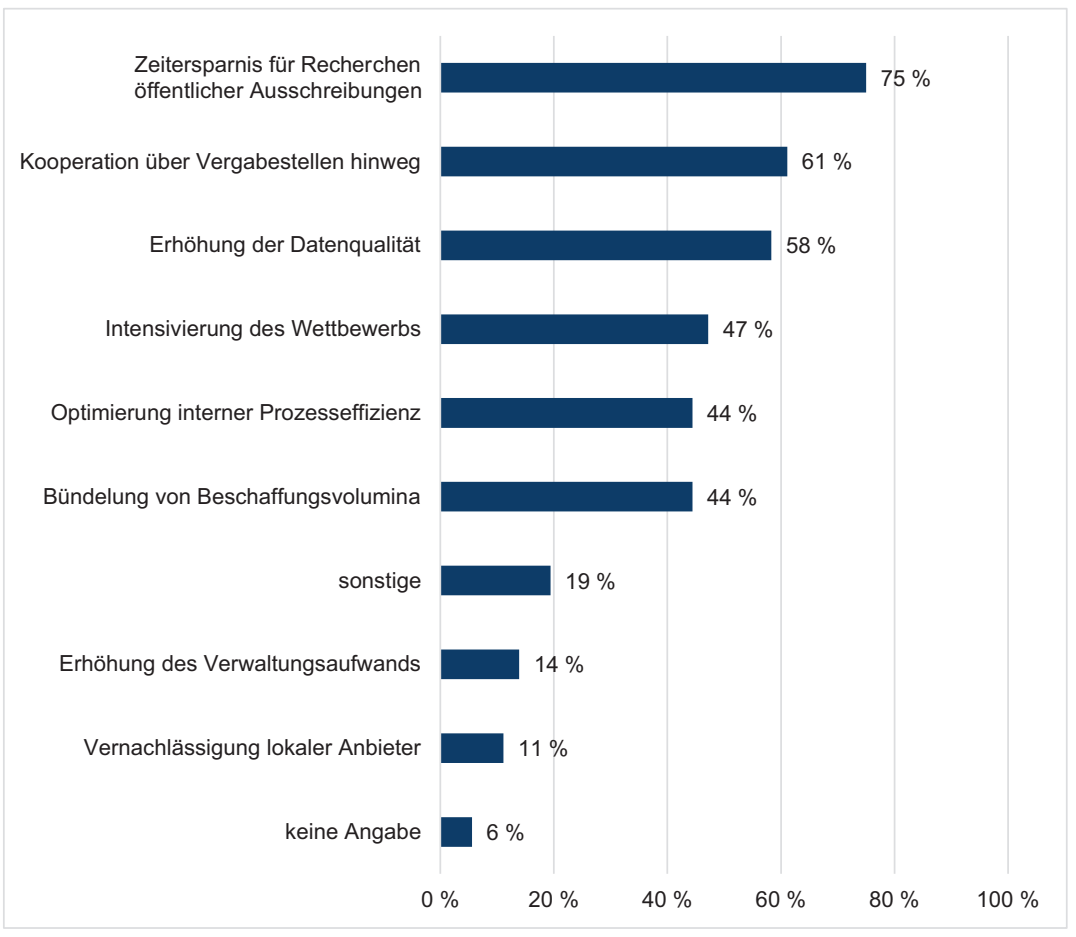

Abbildung 8.19 Wirtschaftliche Folgen der Bereitstellung offener öffentlicher Einkaufsdaten über govdata.de 
Tabelle 8.31 Wirtschaftliche Folgen der Bereitstellung offener öffentlicher Einkaufsdaten über govdata.de

\begin{tabular}{l|c|c}
\hline Antworten & Prozent & Anzahl \\
\hline $\begin{array}{l}\text { Zeitersparnis für Recherchen öffentlicher } \\
\text { Ausschreibungen }\end{array}$ & $75 \%$ & 27 \\
\hline Kooperation über Vergabestellen hinweg & $61 \%$ & 22 \\
\hline Erhöhung der Datenqualität & $58 \%$ & 21 \\
\hline Intensivierung des Wettbewerbs & $47 \%$ & 17 \\
\hline Bündelung von Beschaffungsvolumina & $44 \%$ & 16 \\
\hline Optimierung interner Prozesseffizienz & $44 \%$ & 16 \\
\hline sonstige & $19 \%$ & 7 \\
\hline Erhöhung des Verwaltungsaufwands & $14 \%$ & 5 \\
\hline Vernachlässigung lokaler Anbieter & $11 \%$ & 4 \\
\hline keine Angabe & $6 \%$ & 2 \\
\hline Summe &.$\%$ & $\mathbf{1 3 7}$ \\
\hline Mittelwert & $\mathbf{3 8 \%}$ & $\mathbf{1 4}$ \\
\hline
\end{tabular}

\section{Bewertung:}

Auf die Frage (Mehrfachauswahl), welche wirtschaftlichen Konsequenzen die Bereitstellung offener öffentlicher Einkaufsdaten über den govdata.de-Verbund hätte, nennen gemäß Abbildung 8.19/Tabelle 8.31 drei Viertel der Befragten die Zeitersparnis für Recherchen öffentlicher Ausschreibungen. $61 \%$ sehen als positiven wirtschaftlichen Effekt die Kooperation über Vergabestellen hinweg, $58 \%$ die Erhöhung der Datenqualität sowie $47 \%$ die Intensivierung des Wettbewerbs. Eine Erhöhung des Verwaltungsaufwands sehen nur $14 \%$ als wirtschaftliche Konsequenz, die Vernachlässigung lokaler Anbieter nur $11 \%$.

Unter „sonstige“ äußern sich $19 \%$ der Befragten - hier werden nochmals Transparenz und die positiven Effekte hinsichtlich der Reduktion von Korruption, von schlechter Planung und von Verwaltungsaufwand bei gleichzeitiger Erhöhung von Beteiligung und Akzeptanz erwähnt.

Interessant ist, dass die oftmals gegen die Öffnung öffentlicher Einkaufsdaten angeführten Befürchtungen, der Verwaltungsaufwand sei hoch und lokale KMUs würden benachteiligt, von den Teilnehmern dieser Studie nicht betont werden. 


\section{Frage 19: Welche Anwendungen, Berichte oder Visualisierungen wären zukünftig aus Ihrer Sicht sinnvoll?}

\section{Bewertung:}

Die Frage (offene Nennungen), welche Anwendungen, Berichte oder Visualisierungen künftig sinnvoll wären, veranlasste zahlreiche Angaben und eine hohe Beteiligung. Nach einer Clusterung der Themen in Tabelle 8.32 ergeben sich die Kategorien Datenbereitstellung, Statistiken/Benchmarking, Datenverknüpfungen, Inhalte, grafische Darstellungen, interessante Portale und Sonstige.

Die Nennungen zeigen einerseits den Bedarf, andererseits aber auch die Kreativität und das (unternehmerische) Potential im Umfeld öffentlicher Einkaufsdaten deutlich auf. Der Bedarf nach Statistiken, Auswertungsmöglichkeiten und sogar der Verknüpfung öffentlicher Einkaufsdaten mit anderen Daten ist vorhanden. (Letzteres zeigt sich auch im Ergebnis der Frage 21: Mit $47 \%$ Zustimmung wird die Verknüpfung öffentlicher Einkaufsdaten mit anderen Daten als gesellschafts- und wirtschaftspolitischer Impuls gesehen.)

Tabelle 8.32 Sinnvolle Anwendungen aus Sicht der Befragten

\begin{tabular}{l|l}
\hline Datenbereitstellung & Statistiken/Benchmarking \\
\hline $\begin{array}{l}\text { Die Bundesregierung sollte die Daten in } \\
\text { einem leicht zu bearbeitenden Grund- }\end{array}$ & - Informationen als Grundlage für einen \\
format zur Verfügung stellen (zum & Erfahrungsaustausch zwischen Ver- \\
Beispiel mit dem OCDS), sodass andere & gabestellen (wer hat eine vergleichbare \\
Gruppen (wirtschaftsnahe oder zivil- & Leistung in letzter Zeit ausgeschrieben?) \\
gesellschaftliche, Medien) entsprechende & - Benchmarking für Vergabestellen unter- \\
Anwendungen erstellen können. & einander \\
- Eine Datenbank, in der über eine Nutzer- & - Darstellung von Vergabeaktivitäten \\
oberfläche nach Vergabeordnung und & (gegebenenfalls auch zu Zuschlägen), \\
Gewerk differenziert und sowohl & regional, auch über Kartendarstellungen \\
Regionen als auch Monate ausgewählt & - Gesamtstatistiken über verschiedene \\
werden können. Zusätzlich sollten & Warengruppen; Vergabestatistik nach \\
$\begin{array}{l}\text { Mengenangaben möglich sein. } \\
\text { Möglichkeit einer direkten Datenaus- }\end{array}$ & Regionen \\
wertung & Nutzung künstlicher Intelligenz auf Basis \\
- Allgemein verbesserte Transparenz: Ver- & standardisierter Daten, die verdächtige \\
gabeinformationsportale können schneller & Prozesse aufdecken und optimieren \\
und kostengünstiger realisiert werden. & Einige Lageberichte per Interessensgebiet \\
& (Bildung, Gesundheit), könnten auch \\
& von Ministerien erstellt werden. Wichtig \\
& ist, dass die Verwaltung selbst die Daten \\
\hline
\end{tabular}


Tabelle 8.32 (Fortsetzung)

\begin{tabular}{l|l}
\hline Datenverknüpfungen & Auswertungen \\
\hline $\begin{array}{l}\text { - Verknüpfung von Einkaufsdaten mit Haus- } \\
\text { haltsbudgets und Geodaten }\end{array}$ & - Zuschlagshäufungen \\
- Verknüpfung von Ausschreibungen mit & - Preisentwicklungen \\
Vertragsdaten & - Ausgabenanalyse \\
- Verbindung von Daten mit weiteren Daten & - Beschaffungsvolumina \\
(zum Beispiel: wie viele Ausschreibungen & - Rechnungsberichte in elektronischer \\
im Vergleich zur Wirtschaftsleistung, wie & Form \\
viele Aufträge gingen aus Bayern nach & \\
$\begin{array}{l}\text { NRW im Vergleich zum Bruttosozial- } \\
\text { produkt und zur Anzahl der Unter- }\end{array}$ & \\
nehmen?) & \\
- Identifier für Auftraggeber und -nehmer; & \\
Verknüpfung mit Firmendaten & \\
\hline Grafische Darstellungen & Interessante Portale \\
\hline - Baumkarte/Kacheldiagramm & - https://prozorro.gov.ua/ \\
- Zeitleisten & - https://dozorro.org/ \\
- Netzwerkdarstellungen & - https://redflags.eu/ \\
- Punktsystem & - https://opentender.eu/ \\
- Ampelsystem, rote Flaggen & \\
- Visualisierung des Prozesses von der & \\
Planung bis zur Umsetzung & \\
- Neben Tabellen mehr Grafiken & \\
\hline
\end{tabular}

Frage 20: Welche Lösungen könnten mithilfe offener öffentlicher Einkaufsdaten realisiert - und welche Märkte beziehungsweise Geschäftsmodelle erschlossen werden?

\section{Bewertung:}

Zur Frage (offene Nennungen), welche Lösungen mithilfe offener öffentlicher Einkaufsdaten realisiert und welche Märkte beziehungsweise Geschäftsmodelle erschlossen werden könnten, gibt es ebenfalls zahlreiche Angaben. Nach deren Clusterung in Themengebiete ergeben sich gemäß Tabelle 8.33 Impulse in folgenden Themengebieten: Wirtschaft, Wissenschaft, Verwaltung, Öffentlichkeit, Systeme/Tools, Transparenz/Korruptionsreduktion, Innovation und Märkte sowie Allgemeines. 
Tabelle 8.33 Geschäftsmodelle und Lösungsansätze aus Sicht der Befragten

\begin{tabular}{|c|c|c|}
\hline $\begin{array}{l}\text { Transparenz/Korruptions- } \\
\text { reduktion }\end{array}$ & Systeme/Tools & Allgemeines \\
\hline $\begin{array}{l}\text { - Stärkung der Transparenz, } \\
\text { Rechenschaftspflicht und } \\
\text { Integrität öffentlicher } \\
\text { Aufträge; geringere } \\
\text { Informationsasymmetrien } \\
\text { am Markt } \\
\text { - Höhere Transparenz im } \\
\text { Verteidigungssektor } \\
\text { - Verringerung von } \\
\text { Korruption und Vettern- } \\
\text { wirtschaft, dadurch } \\
\text { Verringerung der Kosten, } \\
\text { Erhöhung des Nutzens }\end{array}$ & \begin{tabular}{|l} 
- Startups und KMUs \\
können spezialisierte \\
Anwendungen anbieten, \\
die wiederum Portale für \\
Dienstanbieter stellen, \\
welche bisher nicht im \\
öffentlichen Sektor auf- \\
getreten sind. \\
- Eine Vergabe- und Preis- \\
datenbank könnte erstellt \\
und mit Zusatzdiensten \\
auch vermarktet werden. \\
- Tools zur Unterstützung \\
der Prozessqualität bei \\
standardisierten Leistungen \\
- https://opentender.eu/ \\
- Digitaler Marktplatz in UK
\end{tabular} & $\begin{array}{l}\text { - Matchmaking } \\
\text { - Zusammenwirken für } \\
\text { mehr Gemeinwohl und } \\
\text { Nachhaltigkeit } \\
\text { - Verbesserung des Ver- } \\
\text { trauens der Öffentlichkeit } \\
\text { - Bessere Evaluierungs- } \\
\text { möglichkeiten der } \\
\text { Vergabeverfahren und } \\
\text { Verfahrensregeln } \\
\text { - Überwachung der Wirk- } \\
\text { samkeit der Servicebereit- } \\
\text { stellung } \\
\text { - Flexiblere Vergabe- } \\
\text { lösungen, die komplexere } \\
\text { Probleme transparent } \\
\text { lösen }\end{array}$ \\
\hline Öffentlichkeit & Wirtschaft & Verwaltung \\
\hline $\begin{array}{l}\text { - Einsparungen von Steuer- } \\
\text { geldern durch ein gutes } \\
\text { Preis-Leistungs-Verhältnis } \\
\text { für die öffentlichen Auf- } \\
\text { träge } \\
\text { - Zugang der Bevölkerung } \\
\text { zu besseren öffentlichen } \\
\text { Dienstleistungen und Infra- } \\
\text { strukturen } \\
\text { - Effektiveres Tracking des } \\
\text { Vergabeprozesses durch } \\
\text { Öffentlichkeit und Medien, } \\
\text { besonders bei Projekten, } \\
\text { die sehr öffentlich sind }\end{array}$ & $\begin{array}{l}\text { - Verbesserung des Zugangs } \\
\text { für KMUs } \\
\text { - Leichtere Recherche von } \\
\text { Vergabeverfahren } \\
\text { - Ermöglichung eines } \\
\text { fairen Wettbewerbs um } \\
\text { öffentliche Aufträge } \\
\text { - Geringere Kosten } \\
\text { - Verbesserung von } \\
\text { Prozessen bis hin zu } \\
\text { Industrie } 4.0\end{array}$ & $\begin{array}{l}\text { - Für die Vergabestellen: } \\
\text { Hilfestellung bei der } \\
\text { Konzeption von Ver- } \\
\text { gabeverfahren und der } \\
\text { Erstellung von Vergabe- } \\
\text { unterlagen } \\
\text { - Standardisierung von Aus- } \\
\text { schreibungstexten } \\
\text { - Zusammenschluss mit } \\
\text { anderen Kommunen } \\
\text { - Bessere Evaluierungs- } \\
\text { möglichkeiten der } \\
\text { Vergabeverfahren und } \\
\text { Verfahrensregeln }\end{array}$ \\
\hline Wissenschaft & Innovationen & Märkte \\
\hline $\begin{array}{l}\text { - Bessere Recherche von } \\
\text { Daten }\end{array}$ & $\begin{array}{l}\text { - Stärkung der Innovationen } \\
\text { durch bessere Analyse und } \\
\text { gezielteren Einkauf } \\
\text { - Ein breiteres Spektrum } \\
\text { innovativer Lösungen } \\
\text { - Größere Diversifizierung } \\
\text { des Marktes - sowohl } \\
\text { durch große als auch } \\
\text { neue Unternehmen mit } \\
\text { Innovationen }\end{array}$ & $\begin{array}{l}\text { - Gesundheit: Bessere, } \\
\text { individualisierte Patienten- } \\
\text { versorgung } \\
\text { - Bessere Berücksichtigung } \\
\text { ökologischer Aspekte } \\
\text { - Energie: Einsparungen } \\
\text { - Verkehr: Logistik, öko- } \\
\text { logisch nachhaltige Ver- } \\
\text { kehrsplanung }\end{array}$ \\
\hline
\end{tabular}


Frage 21: Welche wirtschaftlichen, sozialen oder politischen Impulse können Sie sich durch eine Öffnung öffentlicher Einkaufsdaten vorstellen?

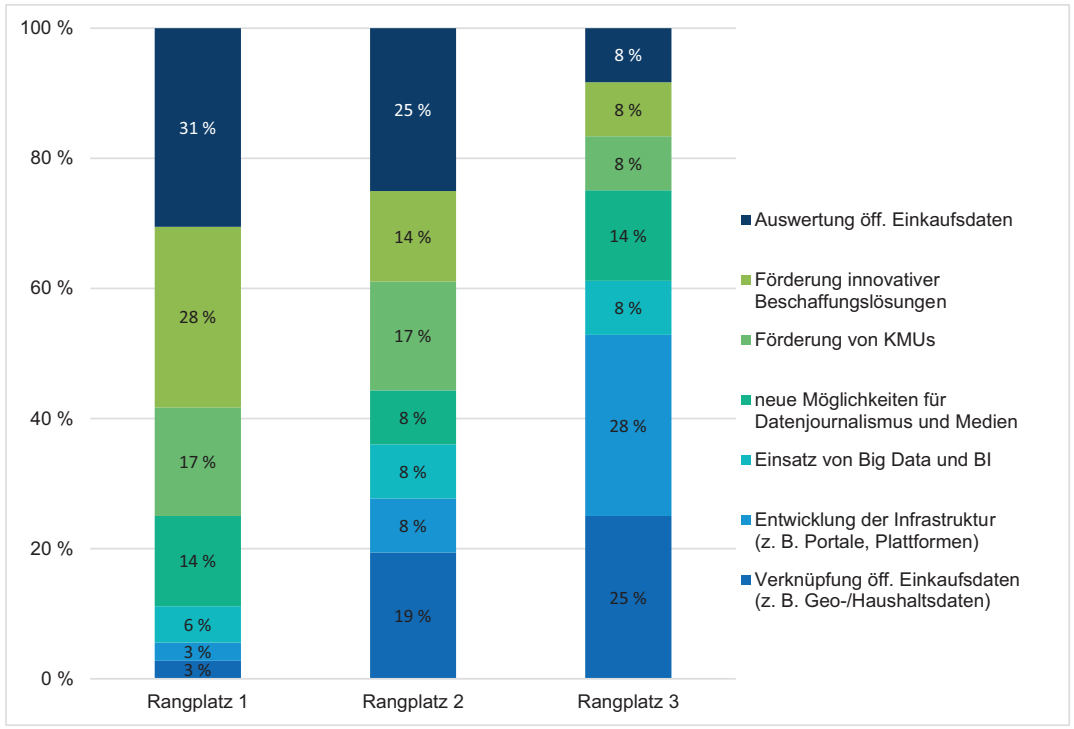

Abbildung 8.20 Impulse aus der Öffnung öffentlicher Einkaufsdaten

Tabelle 8.34 Impulse aus der Öffnung öffentlicher Einkaufsdaten

\begin{tabular}{|c|c|c|c|c|c|c|c|}
\hline \multirow[t]{2}{*}{ Antworten } & \multicolumn{2}{|c|}{$\begin{array}{c}\text { Rangplatz } \\
1\end{array}$} & \multicolumn{2}{|c|}{$\begin{array}{c}\text { Rangplatz } \\
2\end{array}$} & \multicolumn{2}{|c|}{$\begin{array}{c}\text { Rangplatz } \\
\mathbf{3}\end{array}$} & \multirow[b]{2}{*}{$\sum$} \\
\hline & $\%$ & Anz. & $\%$ & Anz. & $\%$ & Anz. & \\
\hline Auswertung öff. Einkaufsdaten & $31 \%$ & 11 & $25 \%$ & 9 & $8 \%$ & 3 & $64 \%$ \\
\hline Förderung innovat. Vergabelösungen & $28 \%$ & 10 & $14 \%$ & 5 & $8 \%$ & 3 & $50 \%$ \\
\hline Förderung von KMUs & $17 \%$ & 6 & $17 \%$ & 6 & $8 \%$ & 3 & $42 \%$ \\
\hline Datenjournalismus und Medien & $14 \%$ & 5 & $8 \%$ & 3 & $14 \%$ & 5 & $36 \%$ \\
\hline Einsatz von Big Data und BI & $6 \%$ & 2 & $8 \%$ & 3 & $8 \%$ & 3 & $22 \%$ \\
\hline Verknüpfung öff. Einkaufsdaten & $3 \%$ & 1 & $19 \%$ & 7 & $25 \%$ & 9 & $47 \%$ \\
\hline Entwicklung der Infrastruktur & $3 \%$ & 1 & $8 \%$ & 3 & $28 \%$ & 10 & $39 \%$ \\
\hline Summe &.$\%$ & 36 &.$\%$ & 36 &.$\%$ & 36 &.$\%$ \\
\hline Mittelwert &.$\%$ &.$\%$ &.$\%$ &.$\%$ &.$\%$ &.$\%$ & $43 \%$ \\
\hline
\end{tabular}




\section{Bewertung:}

Als wesentlicher Impuls aus der Öffnung öffentlicher Einkaufsdaten wird auf Platz eins mit $64 \%$ die Auswertung öffentlicher Einkaufsdaten gesehen, gefolgt von der Förderung innovativer Beschaffungslösungen auf Platz zwei mit 50\% und auf Platz drei mit $47 \%$ die Verknüpfung öffentlicher Einkaufsdaten mit anderen Daten. Am geringsten wird mit $22 \%$ der Einsatz von Big Data und Business Intelligence als Impuls gesehen (siehe Abbildung 8.20/Tabelle 8.34).

\section{Frage 22: Wer könnte welchen Nutzen aus offenen öffentlichen Einkaufs- daten ziehen?}

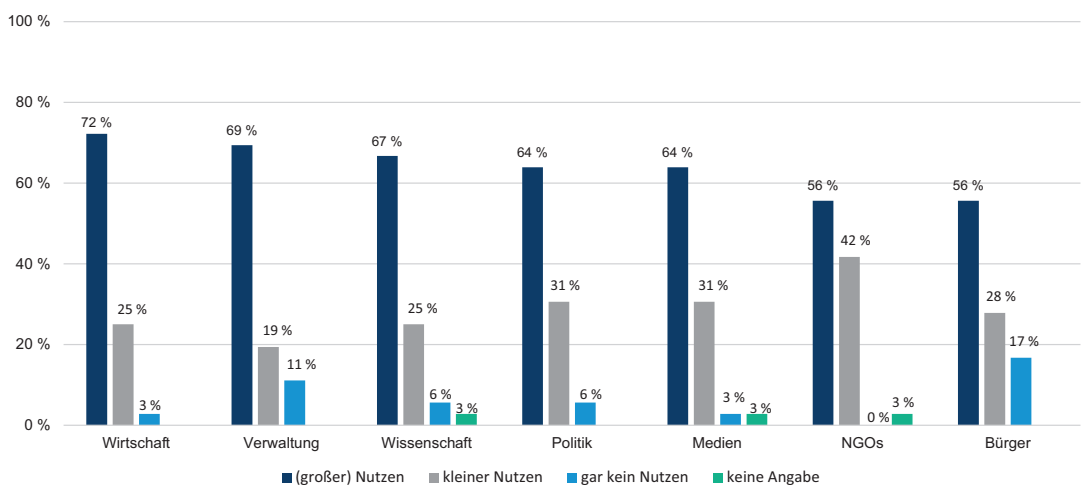

Abbildung 8.21 Nutzen der Öffnung öffentlicher Einkaufsdaten

Tabelle 8.35 Nutzen der Öffnung öffentlicher Einkaufsdaten

\begin{tabular}{l|c|c|c|c|c|c|c|c}
\hline \multirow{2}{*}{ Antworten } & \multicolumn{2}{|c|}{ Großer Nutzen } & \multicolumn{2}{c|}{ Kl. Nutzen } & \multicolumn{2}{c|}{ Kein Nutzen } & \multicolumn{2}{c}{ k. A. } \\
\cline { 2 - 9 } & $\boldsymbol{\%}$ & Anz. & $\boldsymbol{\%}$ & Anz. & $\boldsymbol{\%}$ & Anz. & $\boldsymbol{\%}$ & Anz. \\
\hline Wirtschaft & $72 \%$ & 26 & $25 \%$ & 9 & $3 \%$ & 1 & $0 \%$ & 0 \\
\hline Verwaltung & $69 \%$ & 25 & $19 \%$ & 7 & $11 \%$ & 4 & $0 \%$ & 0 \\
\hline Wissenschaft & $67 \%$ & 24 & $25 \%$ & 9 & $6 \%$ & 2 & $3 \%$ & 1 \\
\hline Politik & $64 \%$ & 23 & $31 \%$ & 11 & $6 \%$ & 2 & $0 \%$ & 0 \\
\hline Medien & $64 \%$ & 23 & $31 \%$ & 11 & $3 \%$ & 1 & $3 \%$ & 1 \\
\hline NGOs & $56 \%$ & 20 & $42 \%$ & 15 & $0 \%$ & 0 & $3 \%$ & 1 \\
\hline Bürger & $56 \%$ & 20 & $28 \%$ & 10 & $17 \%$ & 6 & $0 \%$ & 0 \\
\hline Summe &.$\%$ & $\mathbf{1 6 1}$ &. & $\mathbf{7 2}$ &.$/$ & $\mathbf{1 6}$ &. & $\mathbf{3}$ \\
\hline Mittelwert & $\mathbf{6 4 \%}$ & $\mathbf{2 3}$ & $\mathbf{2 9} \%$ & $\mathbf{1 0}$ & $\mathbf{6 \%}$ & $\mathbf{2}$ & $\mathbf{1 \%}$ & $\mathbf{0}$ \\
\hline
\end{tabular}




\section{Bewertung:}

Auf die Frage, wer welchen Nutzen aus der Öffnung der öffentlichen Einkaufsdaten ziehen könnte, werden, wie Abbildung 8.21/Tabelle 8.35 widerspiegelt, unter "Großer Nutzen“ die Wirtschaft, Verwaltung und Wissenschaft als Gruppen mit der höchsten Zustimmung gesehen (72\%, $69 \%$ und $67 \%$ respektive). Politik und Medien liegen mit $64 \%$ im Bereich des Mittelwerts. Mit immer noch $56 \%$, aber knapp unter dem Mittelwert, werden jeweils Bürger und NGOs genannt.

Unter „Kleiner Nutzen“ sind die größte benannte Gruppe die der NGOs mit $42 \%$ Zustimmung. Unter „Kein Nutzen“ ist die größte benannte Gruppe die der Bürger mit $17 \%$.

\section{Frage 23: Welche Stärken sehen Sie in der (weiteren) Öffnung öffentlicher Einkaufsdaten?}

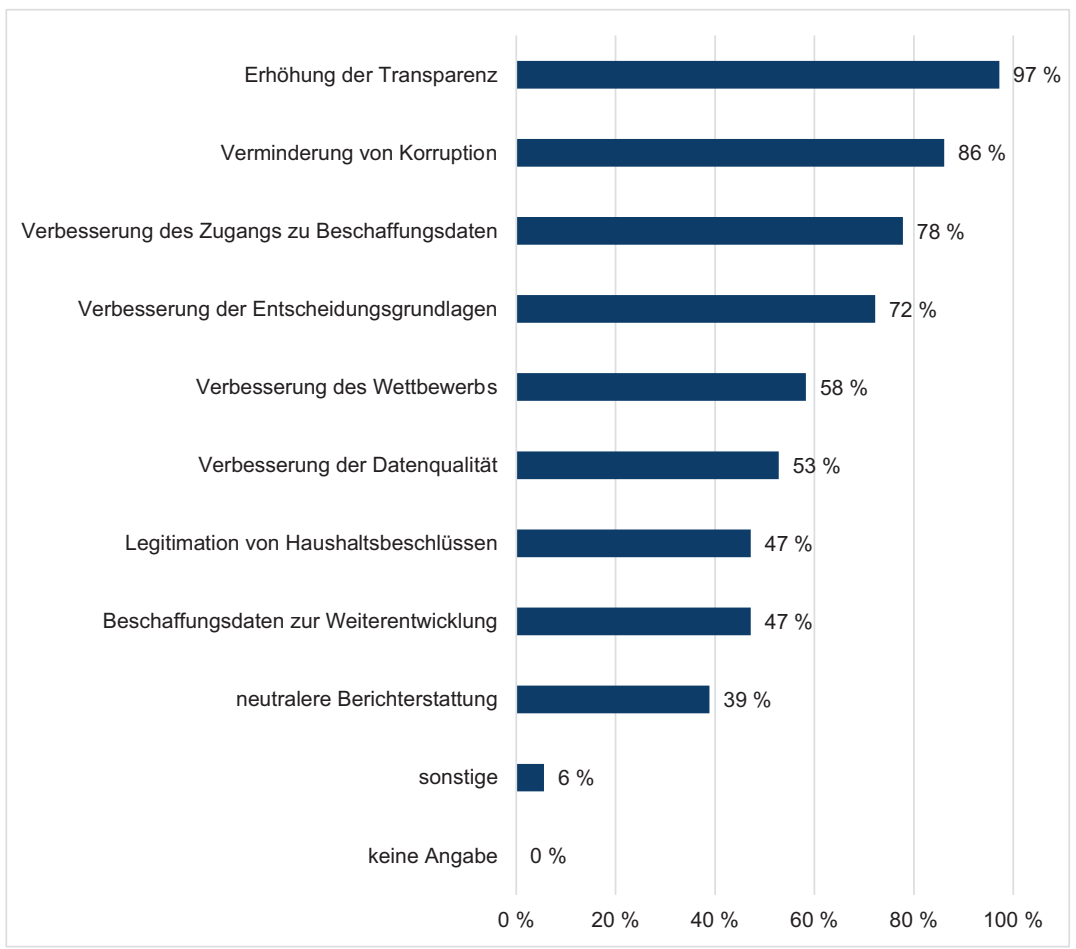

Abbildung 8.22 Stärken der Öffnung öffentlicher Einkaufsdaten 
Tabelle 8.36 Stärken der Öffnung öffentlicher Einkaufsdaten

\begin{tabular}{l|c|c}
\hline Antworten & Prozent & Anzahl \\
\hline Erhöhung der Transparenz & $97 \%$ & 35 \\
\hline Verminderung von Korruption & $86 \%$ & 31 \\
\hline $\begin{array}{l}\text { Verbesserung des Zugangs zu öffentlichen } \\
\text { Einkaufsdaten }\end{array}$ & $\mathbf{7 8 \%}$ & 28 \\
\hline Verbesserung der Entscheidungsgrundlagen & $72 \%$ & 26 \\
\hline Verbesserung des Wettbewerbs & $58 \%$ & 21 \\
\hline Verbesserung der Datenqualität & $53 \%$ & 19 \\
\hline Öffentliche Einkaufsdaten zur Weiter- & $47 \%$ & 17 \\
\hline entwicklung & $47 \%$ & 17 \\
\hline Legitimation von Haushaltsbeschlüssen & $39 \%$ & 2 \\
\hline neutralere Berichterstattung & $6 \%$ & 0 \\
\hline sonstige & $0 \%$ & $\mathbf{2 1 0}$ \\
\hline keine Angabe & $\mathbf{1 9}$ & \\
\hline Summe & $\mathbf{5 3 \%}$ & 19 \\
\hline Mittelwert & & \\
\hline
\end{tabular}

\section{Bewertung:}

Bei der Bewertung der Stärken der Öffnung der öffentlichen Einkaufsdaten (Mehrfachauswahl) laut Abbildung 8.22/Tabelle 8.36 wird mit $97 \%$ klar die Erhöhung der Transparenz, gefolgt von der Verminderung der Korruption (86\%) gesehen. Die Zustimmungen im Mittelfeld rangieren zwischen $47 \%$ und $58 \%-$ sie betreffen die Aspekte Verbesserung des Wettbewerbs, der Datenqualität, der öffentlichen Einkaufsdaten zur Weiterentwicklung und die Legitimation von Haushaltsbeschlüssen. Mit $39 \%$ und deutlich unterhalb des Mittelwerts wird eine neutralere Berichterstattung nicht als wesentliche Stärke der Öffnung der Einkaufsdaten angesehen.

In den Nennungen unter "sonstige“ wird noch auf die Verbesserung und Vereinfachung der Durchführung von Vergabeverfahren sowie mehr Bürgerbeteiligung in (ausgewählten) Planungsverfahren verwiesen (beispielsweise in den Projekten „Stuttgart 21“ oder „Berliner Flughafen“). 


\section{Frage 24: Welche Schwächen sehen Sie in der (weiteren) Öffnung öffentlicher Einkaufsdaten?}

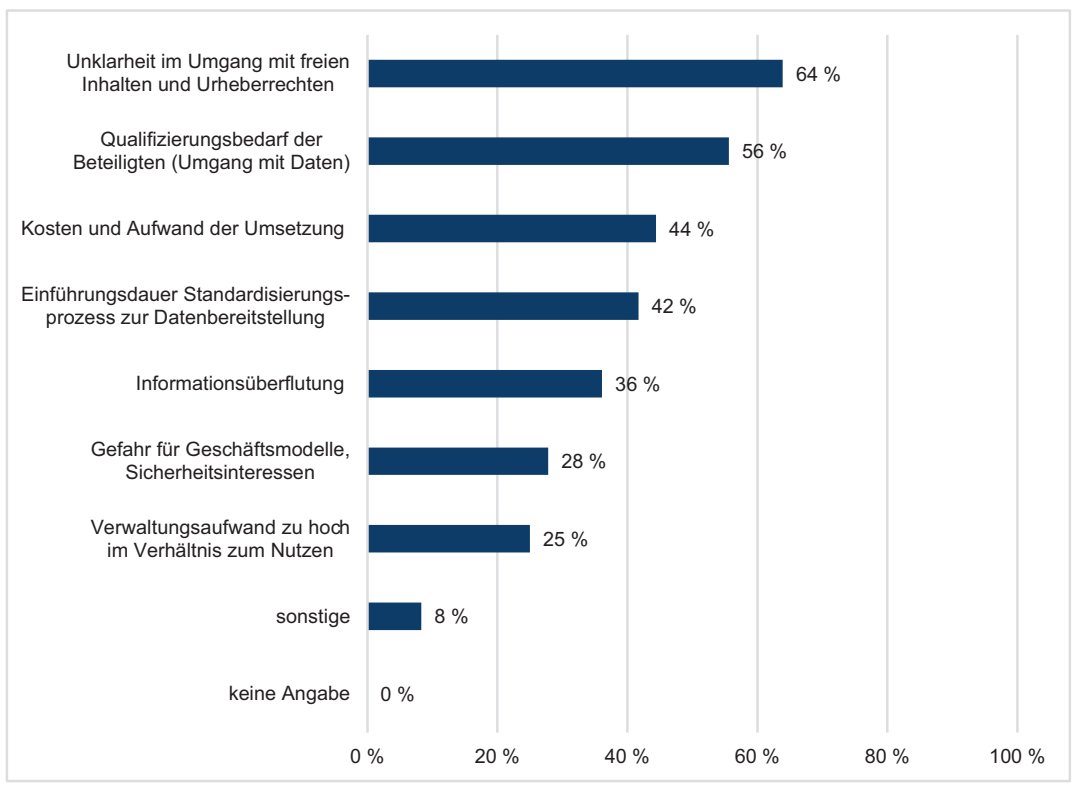

Abbildung 8.23 Schwächen der Öffnung öffentlicher Einkaufsdaten

Tabelle 8.37 Schwächen der Öffnung öffentlicher Einkaufsdaten

\begin{tabular}{l|c|c}
\hline Antworten & Prozent & Anzahl \\
\hline $\begin{array}{l}\text { Unklarheit im Umgang mit freien Inhalten und } \\
\text { Urheberrechten }\end{array}$ & $64 \%$ & 23 \\
\hline $\begin{array}{l}\text { Qualifizierungsbedarf der Beteiligten (Umgang mit } \\
\text { Daten) }\end{array}$ & $56 \%$ & 20 \\
\hline $\begin{array}{l}\text { Kosten und Aufwand der Umsetzung (zum Beispiel } \\
\text { für Datenaufbereitung) }\end{array}$ & $44 \%$ & 16 \\
\hline $\begin{array}{l}\text { Einführungsdauer für Standardisierungsprozess zur } \\
\text { Datenbereitstellung }\end{array}$ & $42 \%$ & 15 \\
\hline \begin{tabular}{l} 
Informationsüberflutung \\
\hline
\end{tabular} & $36 \%$ & 13 \\
\hline
\end{tabular}


Tabelle 8.37 (Fortsetzung)

\begin{tabular}{l|c|c}
\hline Antworten & Prozent & Anzahl \\
\hline Gefahr für Geschäftsmodelle, Sicherheitsinteressen & $28 \%$ & 10 \\
\hline $\begin{array}{l}\text { Verwaltungsaufwand zu hoch im Verhältnis zum } \\
\text { Nutzen }\end{array}$ & $25 \%$ & 9 \\
\hline sonstige & $8 \%$ & 3 \\
\hline keine Angabe & $0 \%$ & 0 \\
\hline Summe &.$/$ & $\mathbf{1 0 9}$ \\
\hline Mittelwert & $\mathbf{3 4 \%}$ & $\mathbf{1 2}$ \\
\hline
\end{tabular}

\section{Bewertung:}

Bei der Bewertung der Schwächen der Öffnung der öffentlichen Einkaufsdaten (Mehrfachauswahl) laut Abbildung 8.23/Tabelle 8.37 wird mit $64 \%$ die Unklarheit im Umgang mit freien Inhalten und Urheberrechten gesehen sowie mit $56 \%$ der Qualifizierungsbedarf der Beteiligten in der Nutzung dieser Daten. Dies ist dicht gefolgt von Kosten und Aufwand der Umsetzung (44\%) sowie der Einführungsdauer für einen Standardisierungsprozess zur Datenbereitstellung (42\%). Mit $25 \%$ und damit der geringsten Zustimmung wird der Verwaltungsaufwand als zu hoch im Verhältnis zum Nutzen eingestuft, gefolgt von $28 \%$ der Befragten, die eine Gefahr für bestehende Geschäftsmodelle oder Sicherheitsinteressen sehen.

Unter "sonstige“ wird nochmals darauf verwiesen, dass die Einführung einer effizienten, transparenten und rechenschaftspflichtigen Vergabepraxis Investitionen in die Qualifizierung von Behördenmitarbeitern, die Verbesserung der Datenqualität und die Anpassung von Prozessen bedeutet, dass dies aber keine Argumente gegen eine Reform im Sinne von Open Contracting sein können. Es wird aber auch befürchtet, dass aus einer Bedarfsbündelung gegebenenfalls ein Verlust von Aufträgen für KMUs resultieren könnte. 


\section{Frage 25: Wo sehen Sie die größten Chancen in der (weiteren) Öffnung öffentlicher Einkaufsdaten?}

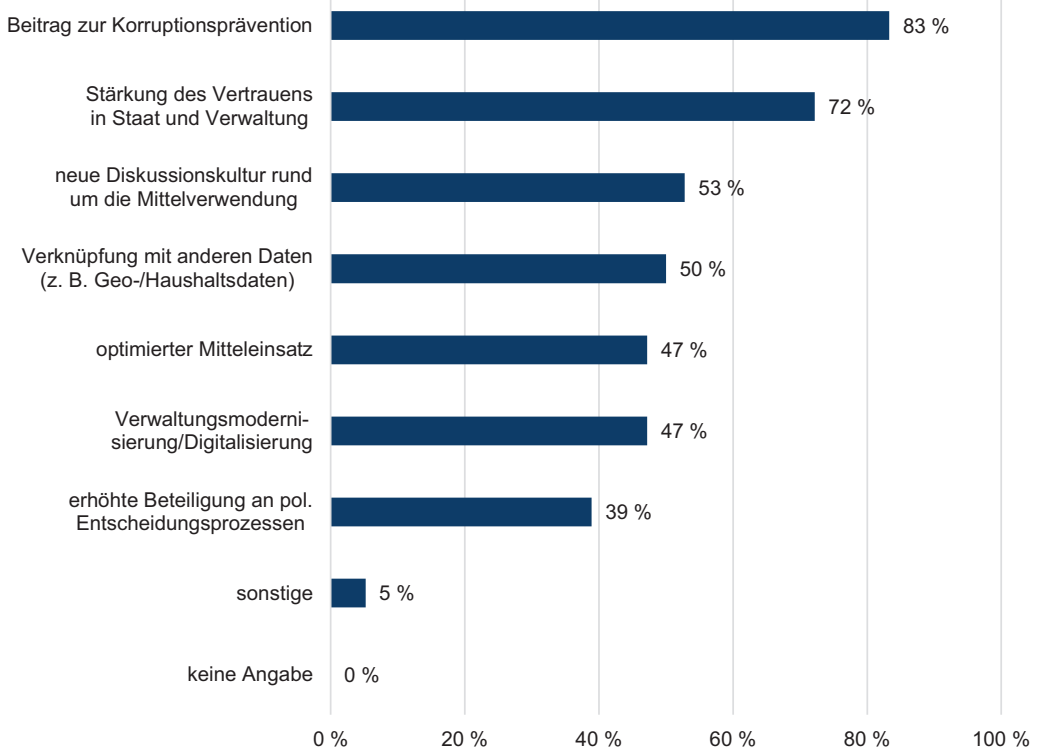

Abbildung 8.24 Chancen der Öffnung öffentlicher Einkaufsdaten

Tabelle 8.38 Chancen der Öffnung öffentlicher Einkaufsdaten

\begin{tabular}{l|c|c}
\hline Antworten & Prozent & Anzahl \\
\hline Beitrag zur Korruptionsprävention & $83 \%$ & 30 \\
\hline Stärkung des Vertrauens in Staat und Verwaltung & $72 \%$ & 26 \\
\hline $\begin{array}{l}\text { Neue Diskussionskultur rund um die Mittelver- } \\
\text { wendung }\end{array}$ & $53 \%$ & 19 \\
\hline $\begin{array}{l}\text { Verknüpfung mit anderen Daten (zum Beispiel } \\
\text { Geo-/Haushaltsdaten) }\end{array}$ & $50 \%$ & 18 \\
\hline optimierter Mitteleinsatz & $47 \%$ & 17 \\
\hline Verwaltungsmodernisierung/Digitalisierung & $47 \%$ & 17 \\
\hline erhöhte Beteiligung an pol. Entscheidungsprozessen & $39 \%$ & 14 \\
\hline sonstige & $5 \%$ & 2 \\
\hline keine Angabe & $0 \%$ & 0 \\
\hline Summe &.$\%$ & $\mathbf{1 4 3}$ \\
\hline Mittelwert & $\mathbf{4 4 \%}$ & $\mathbf{1 6}$ \\
\hline
\end{tabular}




\section{Bewertung:}

Bei der Bewertung der Chancen der Öffnung der öffentlichen Einkaufsdaten (Mehrfachauswahl) gemäß Abbildung 8.24/Tabelle 8.38 wird vor allem der Beitrag zur Korruptionsprävention mit $83 \%$ am höchsten eingestuft - dicht gefolgt von der Stärkung des Vertrauens in Staat und Verwaltung mit $72 \%$. Im Mittelfeld in einer Bandbreite von $47 \%$ bis $53 \%$ Zustimmung rangieren eine „Neue Diskussionskultur rund um die Mittelverwendung“, die „Verknüpfung mit anderen Daten“; ein „optimierter Mitteleinsatz“ sowie „Verwaltungsmodernisierung/ Digitalisierung“. Mit 39\% wird die Chance auf eine erhöhte Beteiligung an politischen Entscheidungsprozessen am geringsten eingestuft.

Frage 26: Welche Risiken sehen Sie in der (weiteren) Öffnung öffentlicher Einkaufsdaten?

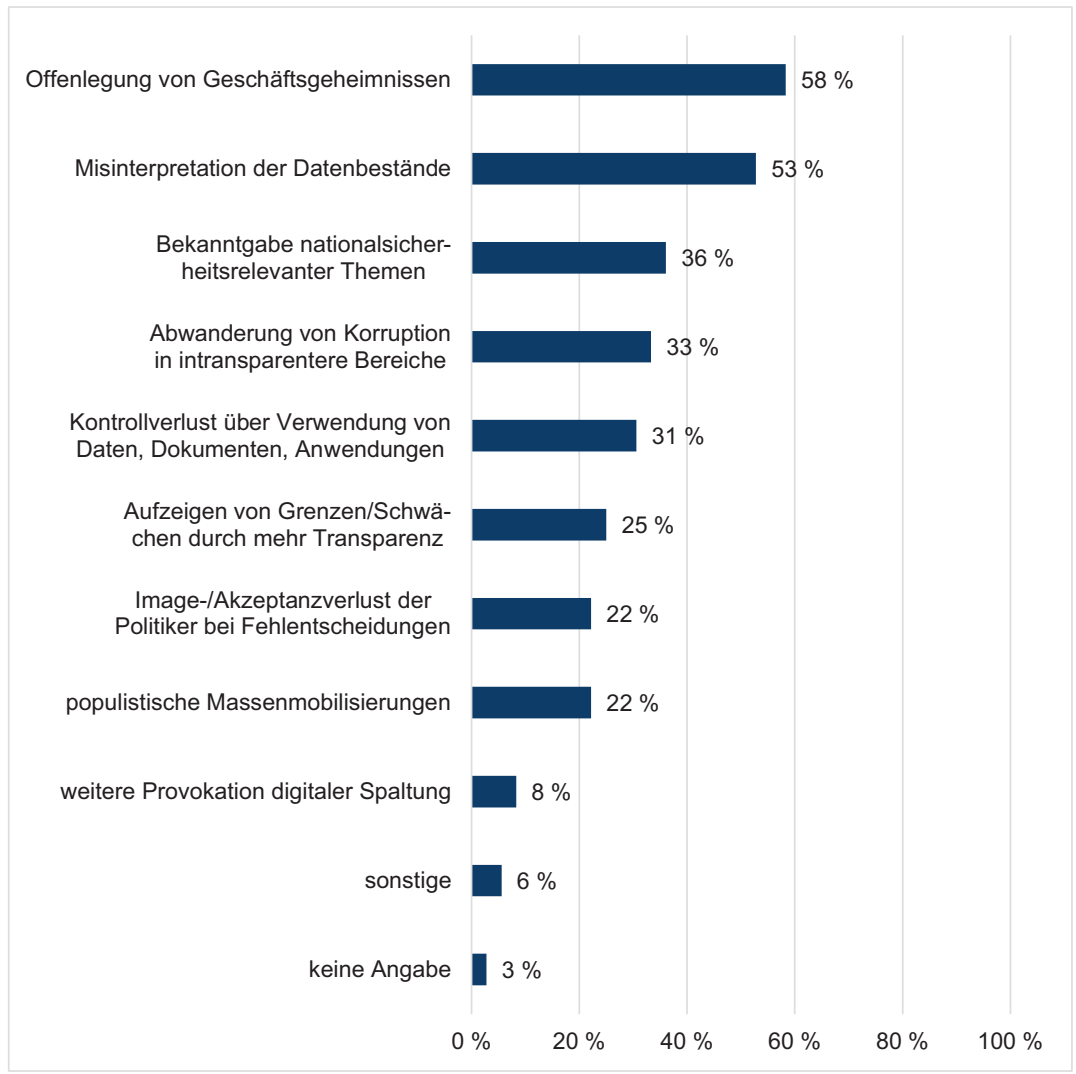

Abbildung 8.25 Risiken der Öffnung öffentlicher Einkaufsdaten 
Tabelle 8.39 Risiken der Öffnung öffentlicher Einkaufsdaten

\begin{tabular}{l|c|c}
\hline Antworten & Prozent & Anzahl \\
\hline Offenlegung von Geschäftsgeheimnissen & $58 \%$ & 21 \\
\hline Fehlinterpretation der Datenbestände & $53 \%$ & 19 \\
\hline Bekanntgabe nationalsicherheitsrelevanter Themen & $36 \%$ & 13 \\
\hline $\begin{array}{l}\text { Abwanderung von Korruption in intransparentere } \\
\text { Bereiche }\end{array}$ & $33 \%$ & 12 \\
\hline $\begin{array}{l}\text { Kontrollverlust über Verwendung von Daten, } \\
\text { Dokumenten, Anwendungen }\end{array}$ & $31 \%$ & 9 \\
\hline $\begin{array}{l}\text { Aufzeigen von Grenzen/Schwächen durch mehr } \\
\text { Transparenz }\end{array}$ & $25 \%$ & 8 \\
\hline Populistische Massenmobilisierungen & $22 \%$ & 8 \\
\hline $\begin{array}{l}\text { Image/Akzeptanzverlust der Politiker bei } \\
\text { Fehlentscheidungen }\end{array}$ & $22 \%$ & 3 \\
\hline weitere Provokation digitaler Spaltung & $8 \%$ & 2 \\
\hline sonstige & $6 \%$ & 1 \\
\hline keine Angabe & $3 \%$ & $\mathbf{1 0 7}$ \\
\hline Summe & $\mathbf{2 7 \%}$ & $\mathbf{1 0}$ \\
\hline Mittelwert & & 9 \\
\hline
\end{tabular}

\section{Bewertung:}

Bei der Bewertung der Risiken der Öffnung der öffentlichen Einkaufsdaten (Mehrfachauswahl) gemäß Abbildung 8.25/Tabelle 8.39 wird vor allem die Offenlegung von Geschäftsgeheimnissen mit $58 \%$ sowie die Fehlinterpretation der Datenbestände mit $53 \%$ eingestuft. Das geringste Risiko wird mit $8 \%$ in der weiteren Provokation einer digitalen Spaltung gesehen (kein Datenzugriff für Teile der Bevölkerung aufgrund eines fehlenden Internetzugangs), gefolgt von Image-/Akzeptanzverlust der Politiker bei Fehlentscheidungen (22\%) sowie populistischen Massenmobilisierungen (22\%).

Unter „sonstige“ wird noch geäußert, dass es sich bei den genannten Risikokategorien um Scheinargumente handele, die von der Notwendigkeit und Sinnhaftigkeit überfälliger Reformen ablenken sollten beziehungsweise die Überforderung der Beteiligten mit dem Thema zeige. 


\subsubsection{Rand- und Rahmenbedingungen}

Frage 27: Inwieweit kann die Öffnung der öffentlichen Einkaufsdaten zu mehr Transparenz führen?

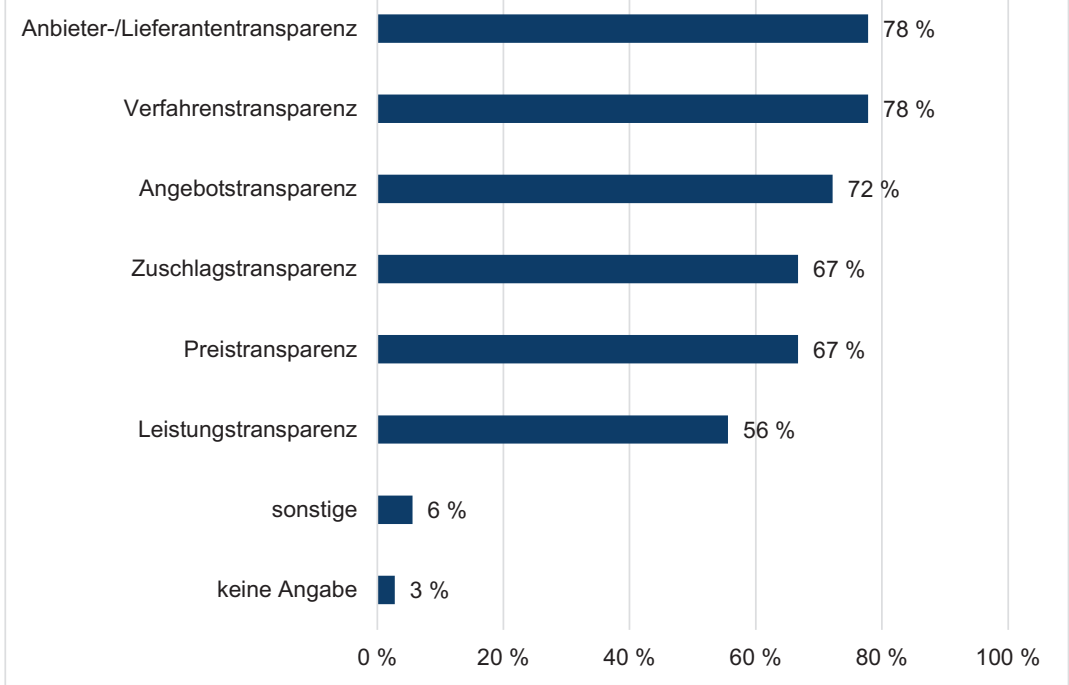

Abbildung 8.26 Auswirkung der Öffnung öffentlicher Einkaufsdaten auf Transparenz

Tabelle 8.40 Auswirkung der Öffnung öffentlicher Einkaufsdaten auf Transparenz

\begin{tabular}{l|c|c}
\hline Antworten & Prozent & Anzahl \\
\hline Anbieter-/Lieferantentransparenz & $78 \%$ & 28 \\
\hline Verfahrenstransparenz & $\mathbf{7 8 \%}$ & 28 \\
\hline Angebotstransparenz & $72 \%$ & 26 \\
\hline Preistransparenz & $67 \%$ & 24 \\
\hline Zuschlagstransparenz & $67 \%$ & 24 \\
\hline Leistungstransparenz & $56 \%$ & 20 \\
\hline sonstige & $6 \%$ & 2 \\
\hline keine Angabe & $3 \%$ & 1 \\
\hline Summe &.$\%$ & $\mathbf{1 5 3}$ \\
\hline Mittelwert & $\mathbf{5 3 \%}$ & $\mathbf{1 9}$ \\
\hline
\end{tabular}




\section{Bewertung:}

Insgesamt wird auf die Frage (Mehrfachauswahl), inwieweit die Öffnung der öffentlichen Einkaufsdaten $\mathrm{zu}$ mehr Transparenz führen kann, laut Abbildung 8.26/Tabelle 8.40 über alle Antwortmöglichkeiten hinweg die Erhöhung der Transparenz deutlich bejaht. Mit $78 \%$ stimmen die Befragten der Verfahrens- und Anbieter-/Lieferantentransparenz zu; Angebots-, Preis- und Zuschlagstransparenz rangieren im Bereich von $67 \%$ bis $72 \%$. Die Leistungstransparenz wird mit $56 \%$ am geringsten bewertet.

Unter ,sonstige“ finden sich noch Verweise auf die Erhöhung der Rechenschaftspflicht als notwendige Voraussetzung sowie auf die Berücksichtigung der Qualität als Leistungsmerkmal im Rahmen der Leistungstransparenz.

Frage 28: Durch welche Maßnahmen in der Beteiligung Dritter kann die Legitimation von öffentlichen Einkaufsentscheidungen in der Zukunft gestützt beziehungsweise verbessert werden?

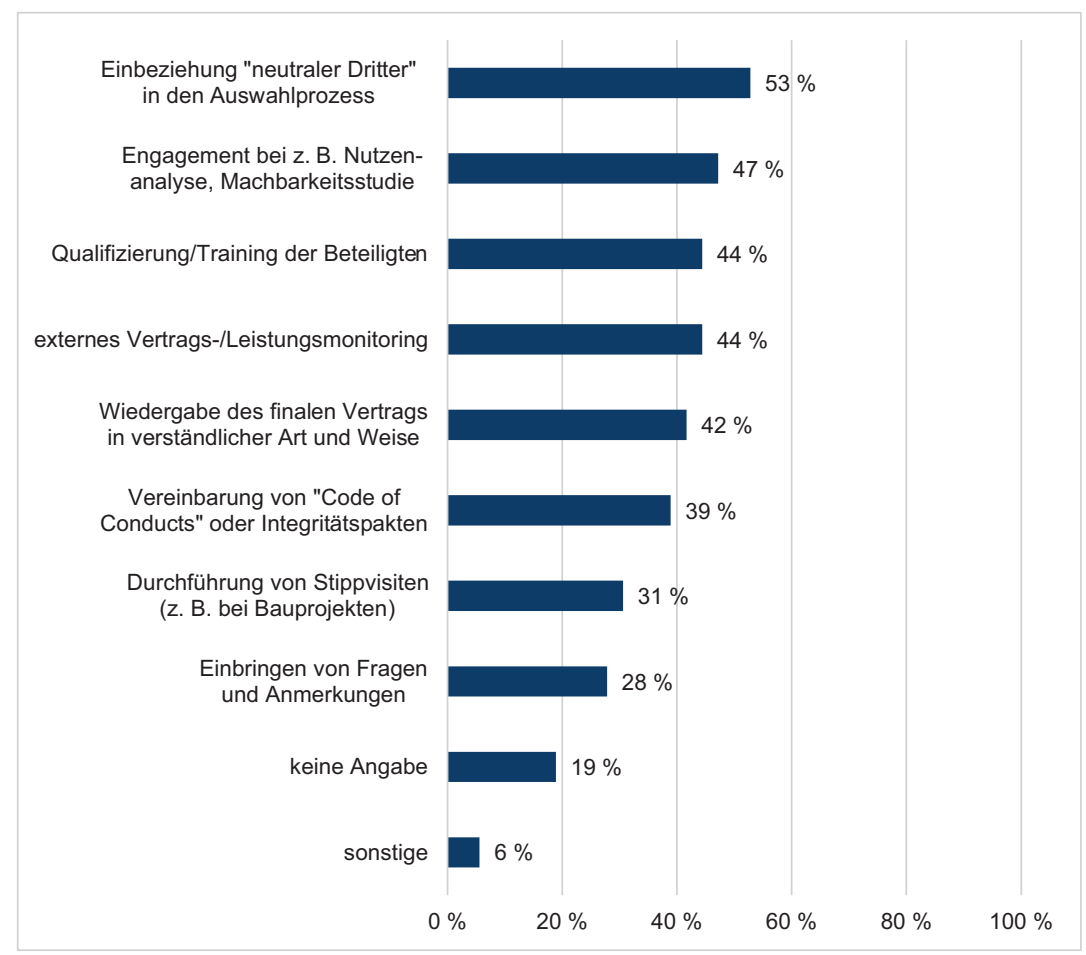

Abbildung 8.27 Maßnahmen zur Beteiligung Dritter 
Tabelle 8.41 Maßnahmen zur Beteiligung Dritter

\begin{tabular}{l|c|c}
\hline Antworten & Prozent & Anzahl \\
\hline $\begin{array}{l}\text { Einbeziehung ,neutraler Dritter“ in den } \\
\text { Auswahlprozess }\end{array}$ & $53 \%$ & 19 \\
\hline $\begin{array}{l}\text { Engagement bei zum Beispiel Nutzenanalyse, } \\
\text { Machbarkeitsstudie }\end{array}$ & $47 \%$ & 17 \\
\hline $\begin{array}{l}\text { externes Vertrags-/ } \\
\text { Leistungsmonitoring }\end{array}$ & $44 \%$ & 16 \\
\hline $\begin{array}{l}\text { Qualifizierung/Training der Beteiligten } \\
\text { Wiedergabe des finalen Vertrags in verständlicher }\end{array}$ & $42 \%$ & 15 \\
\hline $\begin{array}{l}\text { Art und Weise } \\
\text { Vereinbarung von „Code of Conducts“ oder }\end{array}$ & $39 \%$ & 14 \\
Integritätspakten & $31 \%$ & 11 \\
\hline $\begin{array}{l}\text { Durchführung von Stippvisiten (zum Beispiel bei } \\
\text { Bauprojekten) }\end{array}$ & $28 \%$ & 10 \\
\hline Einbringen von Fragen und Anmerkungen & $19 \%$ & 7 \\
\hline keine Angabe & $6 \%$ & 2 \\
\hline sonstige & $\mathbf{3 5 \%}$ & $\mathbf{1 2 7}$ \\
\hline Summe & & $\mathbf{1 3}$ \\
\hline Mittelwert & & \\
\hline
\end{tabular}

\section{Bewertung:}

Auf die Frage (Mehrfachauswahl) nach Maßnahmen in der Beteiligung Dritter zur künftigen Verbesserung der Legitimation öffentlicher Einkaufsentscheidungen führt Abbildung 8.27/Tabelle 8.41 die höchsten Werte der Zustimmung für „Einbeziehung neutraler Dritter in den Auswahlprozess“ (53\%) auf, das „Engagement in frühen Phasen wie der Nutzenanalyse/Machbarkeitsstudie“ (47\%) sowie „Externes Vertrags-/Leistungsmonitoring“ (44\%), welches in den Nennungen identisch mit „Qualifizierung/Training der Beteiligten“ ist (ebenfalls $44 \%$ ). Die geringsten Zustimmungen liegen bei der „Durchführung von Stippvisiten“ (31 \%) und beim „Einbringen von Fragen und Anmerkungen“ (28\%). Bemerkenswert ist der hohe Anteil von „keine Angabe“ mit $19 \%$. Auch hier werden die Resultate der Literaturrecherche durch eine Unterstützung der Einbeziehung Externer untermauert. 
Frage 29: In welchem Teil des Gesamtprozesses der Beschaffung kann Ihrer Meinung nach durch die Öffnung von öffentlichen Einkaufsdaten Korruption am ehesten reduziert werden?

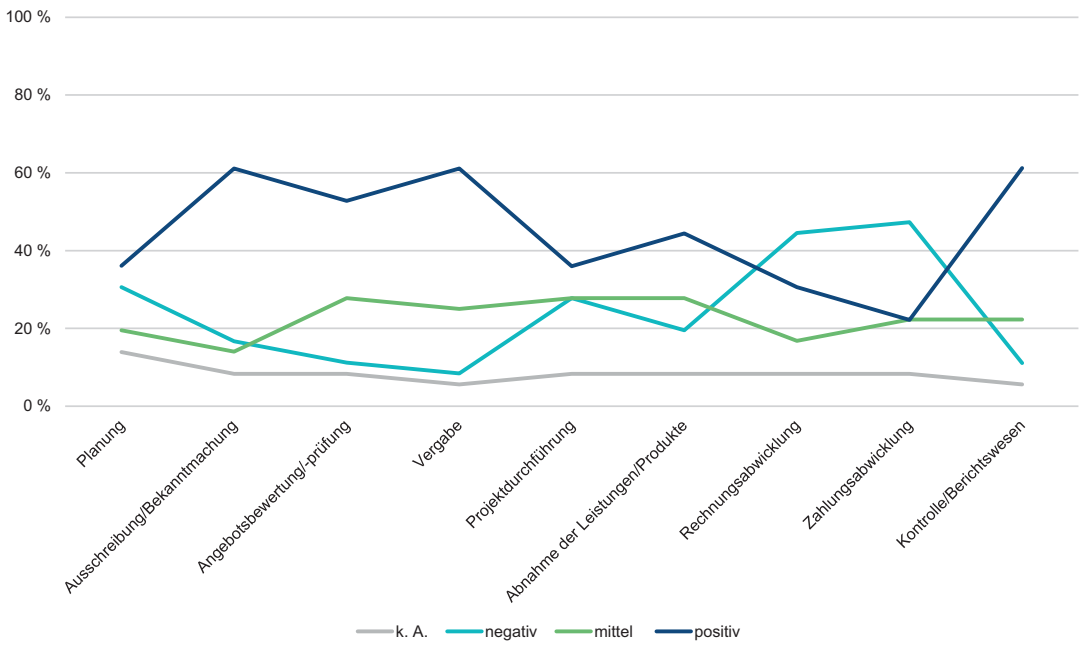

Abbildung 8.28 Einfluss der Öffnung öffentlicher Einkaufsdaten auf Reduktion der Korruption

Tabelle 8.42 Einfluss der Öffnung öffentlicher Einkaufsdaten auf Reduktion der Korruption

\begin{tabular}{l|c|c|c|c|c|c|c|c}
\hline \multirow{2}{*}{ Antworten } & \multicolumn{2}{|c|}{ Positiv } & \multicolumn{2}{c|}{ Mittel } & \multicolumn{2}{c|}{ Negativ } & \multicolumn{2}{c}{ k. A. } \\
\cline { 2 - 10 } & Anz. & $\%$ & Anz. & $\%$ & Anz. & $\%$ & Anz. & $\%$ \\
\hline Planung & 13 & $36 \%$ & 7 & $20 \%$ & 11 & $31 \%$ & 5 & $14 \%$ \\
\hline $\begin{array}{l}\text { Ausschreibung/ } \\
\text { Bekanntmachung }\end{array}$ & 22 & $61 \%$ & 5 & $14 \%$ & 6 & $17 \%$ & 3 & $8 \%$ \\
\hline $\begin{array}{l}\text { Angebotsbewertung/- } \\
\text { prüfung }\end{array}$ & 19 & $53 \%$ & 10 & $28 \%$ & 4 & $11 \%$ & 3 & $8 \%$ \\
\hline Vergabe & 22 & $61 \%$ & 9 & $25 \%$ & 3 & $8 \%$ & 2 & $6 \%$ \\
\hline Projektdurchführung & 13 & $36 \%$ & 10 & $28 \%$ & 10 & $28 \%$ & 3 & $8 \%$ \\
\hline $\begin{array}{l}\text { Abnahme Leistungen/ } \\
\text { Produkte }\end{array}$ & 16 & $44 \%$ & 10 & $28 \%$ & 7 & $20 \%$ & 3 & $8 \%$ \\
\hline
\end{tabular}


Tabelle 8.42 (Fortsetzung)

\begin{tabular}{l|c|c|c|c|c|c|c|c}
\hline \multirow{2}{*}{ Antworten } & \multicolumn{2}{|c|}{ Positiv } & \multicolumn{2}{c|}{ Mittel } & \multicolumn{2}{c|}{ Negativ } & \multicolumn{2}{c}{ k. A. } \\
\cline { 2 - 10 } & Anz. & $\boldsymbol{\%}$ & Anz. & $\boldsymbol{\%}$ & Anz. & $\%$ & Anz. & $\%$ \\
\hline Rechnungsabwicklung & 11 & $31 \%$ & 6 & $17 \%$ & 16 & $45 \%$ & 3 & $8 \%$ \\
\hline Zahlungsabwicklung & 8 & $22 \%$ & 8 & $22 \%$ & 17 & $47 \%$ & 3 & $8 \%$ \\
\hline $\begin{array}{l}\text { Kontrolle/ } \\
\text { Berichtswesen }\end{array}$ & 22 & $61 \%$ & 8 & $22 \%$ & 4 & $11 \%$ & 2 & $6 \%$ \\
\hline Summe & $\mathbf{1 4 6}$ &.$/$ & $\mathbf{7 3}$ &.$/$ & $\mathbf{7 8}$ &.$/$ & $\mathbf{2 7}$ &. \\
\hline Mittelwert & $\mathbf{1 6}$ & $\mathbf{4 5} \%$ & $\mathbf{8}$ & $\mathbf{2 3 \%}$ & $\mathbf{9}$ & $\mathbf{2 4} \%$ & $\mathbf{5}$ & $\mathbf{8 \%}$ \\
\hline
\end{tabular}

\section{Bewertung:}

Wie in Abbildung 8.28/Tabelle 8.42 ersichtlich, gehen $61 \%$ der Teilnehmer davon aus, dass in den Teilprozessen der Ausschreibung/Bekanntmachung, der Vergabe und der Kontrolle/des Berichtswesens durch die Öffnung der öffentlichen Einkaufsdaten Korruption am ehesten reduziert werden kann. Für den Teilprozess der Angebotsbewertung sehen dies noch $53 \%$ der Befragten, für die Phase der Abnahme der Leistungen etwa $44 \%$. Die geringsten Chancen zur Korruptionsreduktion werden bei der Planung (36\%), Projektdurchführung (36\%) und Rechnungs- und Zahlungsabwicklung (31\% respektive $22 \%$ ) gesehen. 
Frage 30: Welche weiteren, flankierenden Maßnahmen sind über die Öffnung des öffentlichen Einkaufswesens hinweg aus Ihrer Sicht notwendig, um Transparenz nachhaltig abzusichern beziehungsweise Korruption einzudämmen?

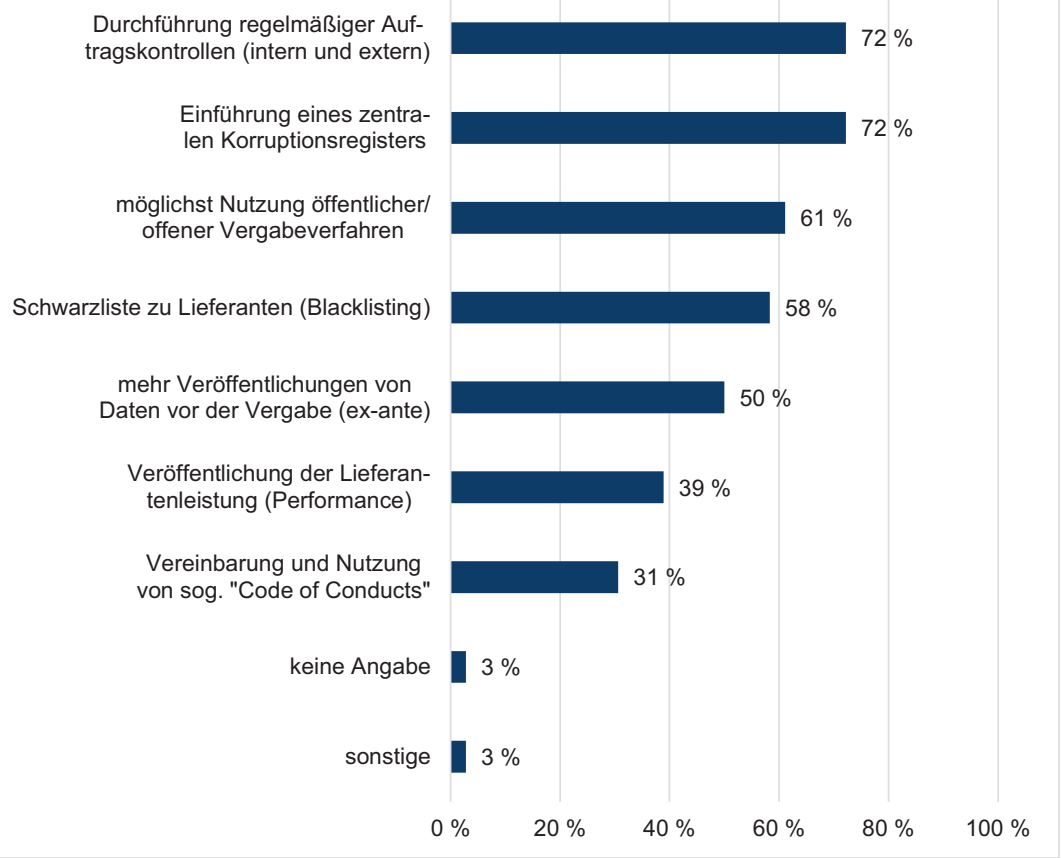

Abbildung 8.29 Weitere Maßnahmen zur Eindämmung der Korruption

Tabelle 8.43 Weitere Maßnahmen zur Eindämmung der Korruption

\begin{tabular}{l|c|c}
\hline Antworten & Prozent & Anzahl \\
\hline $\begin{array}{l}\text { Durchführung regelmäßiger Auftragskontrollen } \\
\text { (intern und extern) }\end{array}$ & $72 \%$ & 26 \\
\hline Einführung eines zentralen Korruptionsregisters & $72 \%$ & 26 \\
\hline $\begin{array}{l}\text { möglichst Nutzung öffentlicher/offener } \\
\text { Vergabeverfahren }\end{array}$ & $61 \%$ & 22 \\
\hline
\end{tabular}

(Fortsetzung) 
Tabelle 8.43 (Fortsetzung)

\begin{tabular}{l|c|c}
\hline Antworten & Prozent & Anzahl \\
\hline Schwarzliste zu Lieferanten (Blacklisting) & $58 \%$ & 21 \\
\hline $\begin{array}{l}\text { mehr Veröffentlichungen von Daten vor der Vergabe } \\
\text { (ex-ante) }\end{array}$ & $50 \%$ & 18 \\
\hline $\begin{array}{l}\text { Veröffentlichung der } \\
\text { Lieferantenleistung (Performance) }\end{array}$ & $39 \%$ & 14 \\
\hline Vereinbarung und Nutzung von „Code of Conducts“ & $31 \%$ & 11 \\
\hline keine Angabe & $3 \%$ & 1 \\
\hline sonstige & $3 \%$ & 1 \\
\hline Summe &.$\%$ & $\mathbf{1 4 0}$ \\
\hline Mittelwert & $\mathbf{4 3 \%}$ & $\mathbf{1 6}$ \\
\hline
\end{tabular}

\section{Bewertung:}

Auf die Frage (Mehrfachauswahl), welche weiteren Maßnahmen über die Öffnung der öffentlichen Einkaufsdaten hinaus notwendig sind, um Transparenz nachhaltig abzusichern beziehungsweise Korruption zu reduzieren, erhalten fünf der sieben Antwortmöglichkeiten Zustimmungswerte von mindestens $50 \%$ (siehe Abbildung 8.29/Tabelle 8.43). Am meisten Zustimmung finden mit jeweils $72 \%$ die Durchführung einer regelmäßigen internen und externen Auftragskontrolle sowie die Einführung eines zentralen Korruptionsregisters. Mit $61 \%$ wird die Nutzung öffentlicher/offener Vergabeverfahren sowie $58 \%$ eine Schwarzliste zu Lieferanten befürwortet. Die Veröffentlichung der Lieferantenleistung erhält hingegen nur $39 \%$ und die Nutzung von „Code of Conducts“ nur $31 \%$ Zustimmung.

Unter „sonstige“ wird noch auf die „Einbeziehung von Nutzern und Betroffenen in Planungsverfahren und die Einrichtung und Nutzung von Feedback-Mechanismen zwischen Öffentlichkeit und Aufsichtsbehörden“"verwiesen.

Auch hier wird - in Ergänzung zu Frage 28 - das Potential durch Kontrollmechanismen, sowohl intern wie auch extern, gesehen. 
Frage 31: Welche Mechanismen eignen sich gut, um das Engagement verschiedener Beteiligter rund um offene öffentliche Einkaufsdaten zu fördern?

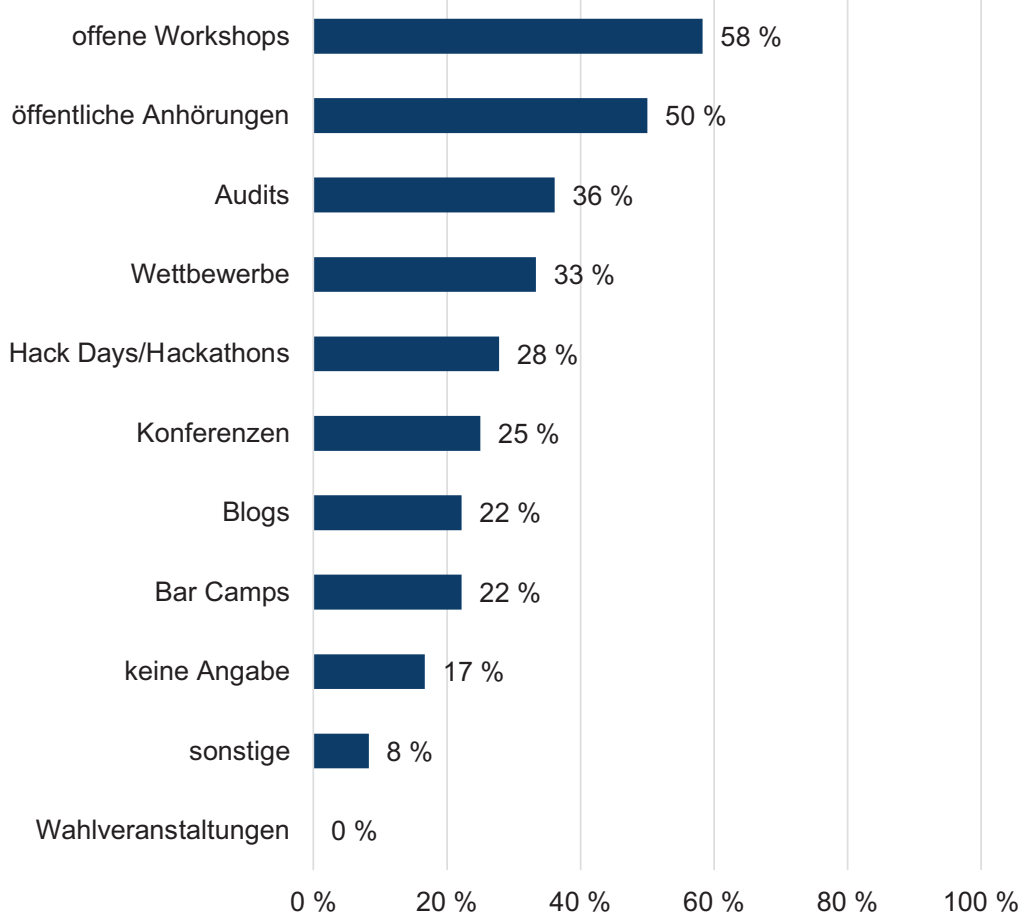

Abbildung 8.30 Mechanismen zur Stärkung des Engagements Dritter 
Tabelle 8.44 Mechanismen zur Stärkung des Engagements Dritter

\begin{tabular}{l|c|c}
\hline Antworten & Prozent & Anzahl \\
\hline offene Workshops & $58 \%$ & 21 \\
\hline öffentliche Anhörungen & $50 \%$ & 18 \\
\hline Audits & $36 \%$ & 13 \\
\hline Wettbewerbe & $33 \%$ & 12 \\
\hline Hack Days/Hackathons & $28 \%$ & 10 \\
\hline Konferenzen & $25 \%$ & 9 \\
\hline Bar Camps & $22 \%$ & 8 \\
\hline Blogs & $22 \%$ & 8 \\
\hline keine Angabe & $17 \%$ & 3 \\
\hline sonstige & $8 \%$ & 0 \\
\hline Wahlveranstaltungen & $0 \%$ & $\mathbf{1 0 8}$ \\
\hline Summe &.$\%$ & $\mathbf{1 0}$ \\
\hline Mittelwert & $\mathbf{2 7 \%}$ & 6 \\
\hline
\end{tabular}

\section{Bewertung:}

Auf die Frage (Mehrfachauswahl), welche Mechanismen sich gut eignen, um das Engagement verschiedener Beteiligter rund um offene öffentliche Einkaufsdaten zu fördern, spricht sich gemäß Abbildung 8.30/Tabelle 8.44 gut die Hälfte für offene Workshops und öffentliche Anhörungen aus (58\% beziehungsweise $50 \%$ ), etwa jeweils ein Drittel für Audits, Wettbewerbe und Hack Days/ Hackathons. Etwa ein Viertel befürwortet Konferenzen, Blogs und Bar Camps. Als völlig ungeeignet werden Wahlveranstaltungen mit $0 \%$ gesehen.

Unter "sonstige“ werden noch Bieterdialoge, normative Handlungsanweisungen und die Einbeziehung von Nutzern und Betroffenen in Planungsverfahren und die Einrichtung und Nutzung von Feedback-Mechanismen zwischen Öffentlichkeit und Aufsichtsbehörden genannt. Deutlich wird insgesamt die Abkehr von traditionellen hin zu den moderneren Formaten der E-/offenen Partizipation (Tabelle 8.45). 
Frage 32: Sind aus Ihrer Sicht zusätzliche Ressourcen (zum Beispiel Mitarbeiter, Budget) zur Öffnung des öffentlichen Einkaufswesens über die vorhandenen Bordmittel hinaus erforderlich?

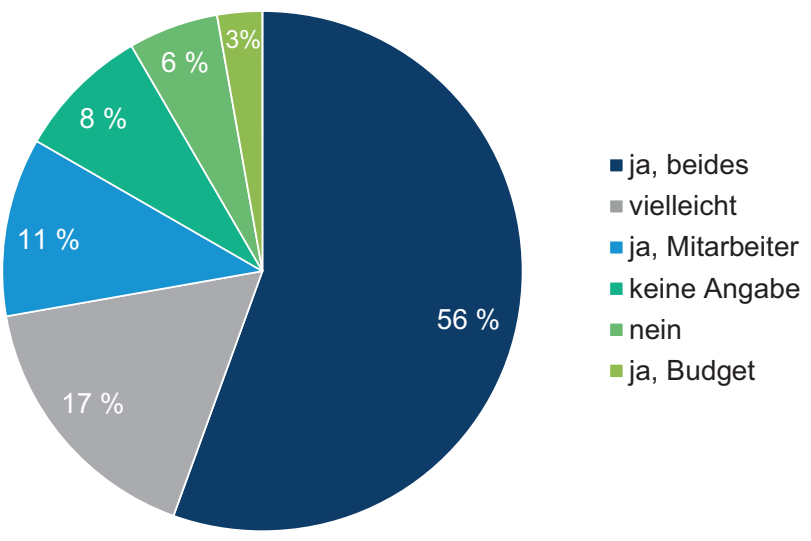

Abbildung 8.31 Benötigte Ressourcen zur Bereitstellung öffentlicher Einkaufsdaten

Tabelle 8.45 Benötigte Ressourcen nach Antworthäufigkeit

\begin{tabular}{l|c|c}
\hline Antworten & Prozent & Anzahl \\
\hline ja, beides & $56 \%$ & 20 \\
\hline vielleicht & $17 \%$ & 6 \\
\hline ja, Mitarbeiter & $11 \%$ & 4 \\
\hline keine Angabe & $8 \%$ & 3 \\
\hline nein & $6 \%$ & 2 \\
\hline ja, Budget & $3 \%$ & 1 \\
\hline Summe & $\mathbf{1 0 0 \%}$ & $\mathbf{3 6}$ \\
\hline
\end{tabular}


Tabelle 8.46 Benötigte Ressourcen nach Antwortreihenfolge

\begin{tabular}{l|c|c}
\hline Antworten & Prozent & Anzahl \\
\hline ja, Budget & $3 \%$ & 1 \\
\hline ja, Mitarbeiter & $11 \%$ & 4 \\
\hline ja, Beides & $56 \%$ & 20 \\
\hline vielleicht & $17 \%$ & 6 \\
\hline nein & $6 \%$ & 2 \\
\hline keine Angabe & $8 \%$ & 3 \\
\hline Summe & $\mathbf{1 0 0 \%}$ & $\mathbf{3 6}$ \\
\hline
\end{tabular}

\section{Bewertung:}

Hinsichtlich der Frage, inwieweit zusätzliche Ressourcen (zum Beispiel Mitarbeiter, Budget) zur Öffnung des öffentlichen Einkaufswesens über die vorhandenen Bordmittel hinaus erforderlich sind, sieht mehr als die Hälfte der Befragten (56\%) die Notwendigkeit, sowohl Budget als auch Mitarbeiter für die weitere Öffnung dieser Daten bereitzustellen. $11 \%$ der Befragten sehen die Notwendigkeit für weiteres Personal - noch vor zusätzlichem Budget (3\% der Befragten). Dies erscheint vor dem Hintergrund, dass zumindest zu Beginn mit erhöhtem Einführungsaufwand zu rechnen ist, realistisch. Unsicher im Sinne von ,vielleicht“ sind $17 \%$ der Befragten. Abbildung 8.31 und Tabelle 8.46 zeigen die Werte.

Frage 33: Was hat sich aus Ihrer Sicht mit der Umsetzung der EUVergaberichtlinien (2014/23/EU, 2014/24/EU und 2014/25/EU) in nationales Recht in 2016/2017 bezüglich der Offenlegung von öffentlichen Einkaufsdaten verändert - was wird sich noch verändern?

\section{Bewertung:}

Auf die Frage (offene Texteingabe), was sich mit der Umsetzung der EU-Vergaberichtlinien in nationales Recht in 2016/2017 bezüglich der Offenlegung von öffentlichen Einkaufsdaten verändert hat, bestätigen die Befragten übereinstimmend, dass es keine wesentlichen Änderungen gegeben habe. Begründet wird dies zum Beispiel damit, dass die im Vergaberecht enthaltenen Bekanntmachungspflichten bereits in der Vergangenheit bestanden hätten und mit der Reform im Wesentlichen der Zugriff auf Ausschreibungsunterlagen im Oberschwellenbereich verbessert worden sei.

Positiv wird angemerkt, dass mit der verpflichtenden eVergabe zumindest die Daten im Oberschwellenbereich einfacher elektronisch erzeugt, strukturiert und 
ausgewertet werden könnten. Dies trägt aus Sicht der Befragten zur erhöhten Transparenz und Rechenschaft für EU-weite Vergabeverfahren bei. Gleichzeitig erfolge so eine Angleichung einiger Vergabemodalitäten in Europa.

Die Befragten adressieren mit Blick auf künftige Veränderungen aber auch ihre Erwartung, dass im Unterschwellenbereich bald ähnlich gehandelt werden möge, sodass auch für diesen die entsprechenden Daten bereitstehen, um eine größere Transparenz und Rechenschaftspflicht zu erzielen.

\section{Frage 34: Was müssten weitere europäische und nationale Gesetzgebungen, Richtlinien und Verordnungen noch beinhalten, um die Vision eines offenen öffentlichen Einkaufs Realität werden zu lassen?}

\section{Bewertung:}

Auf die Frage (offene Texteingabe), was weitere europäische und nationale Gesetzgebungen, Richtlinien und Verordnungen noch beinhalten müssten, um die Vision eines offenen öffentlichen Einkaufs Realität werden zu lassen, wurden vielfältige Antworten gegeben. Diese können in die Kategorien ,keine wesentlichen Änderungen“, „konkrete Erweiterungen“ und „Fokus auf Transparenz und Gemeinwohl" eingeteilt werden.

Unter „keine wesentlichen Änderungen“ wird adressiert, dass es zunächst Umsetzungs- und Vollzugsdefizite zu beseitigen gelte, bevor man weitere Reformen anstrebt.

In die Kategorie „,konkrete Erweiterungen“ fallen die gesetzliche Ausdehnung der eVergabe sowie Datenöffnung entlang des gesamten öffentlichen Einkaufsprozesses von der Bedarfsfeststellung bis hin zur Rechnungsbegleichung, und zwar auch für den Unterschwellenbereich. Zugleich solle man die Resultate der Datenöffnung in Auditprozessen und Jahresabschlüssen verankern. Mit Blick auf den Umfang besteht Uneinigkeit und Diskussionsbedarf: Während einige Teilnehmer von bestimmten Daten sprechen, sprechen andere von einer vollständigen Öffnung. Die PSI-Richtlinie, IFG, IWG und Datenschutz sollten weiter durch die Normgeber präzisiert und verschärft werden. Als gutes Beispiel und möglicherweise Vorlage wird das HmbTG angeführt.

Unter dem „Fokus auf Transparenz und Gemeinwohl“" wird ebenfalls die Öffnung des Gesamtprozesses von der Planung bis zur Leistung des Unternehmens befürwortet und auch, dass das Gemeinwohl Vorrang vor unbegründeten Geheimhaltungsinteressen von Unternehmen haben solle. Die Bedeutung der proaktiven Veröffentlichungspflicht und „open by default“-Standards werden betont. Als konkrete Umsetzungswünsche werden die Einführung des OCDS, öffentlich zugängliche Unternehmens- und Lobbyregister sowie Register zum Staatsbesitz 
genannt. Außerdem werden der gesetzlich und praktisch umzusetzende Schutz der Hinweisgeber, die Nutzung einer Matrix zur Gemeinwohl-Ökonomie, die ein Unternehmen gesamthaft über verschiedene Kriterien hinweg bewertet, sowie eine verbesserte Strafverfolgung bei Missbrauch und Korruption gewünscht. Des Weiteren solle eine Verknüpfung der öffentlichen Einkaufsdaten mit Staatsbesitz und Umweltkriterien erfolgen, um so Zusammenhänge besser erkennen zu können.

Frage 35: Ist das Transparenzgesetz in Hamburg als Vorreiter oder Blaupause aus Ihrer Sicht ein guter Vorreiter und als vorbildhafte Umsetzung geeignet? Warum beziehungsweise warum nicht?

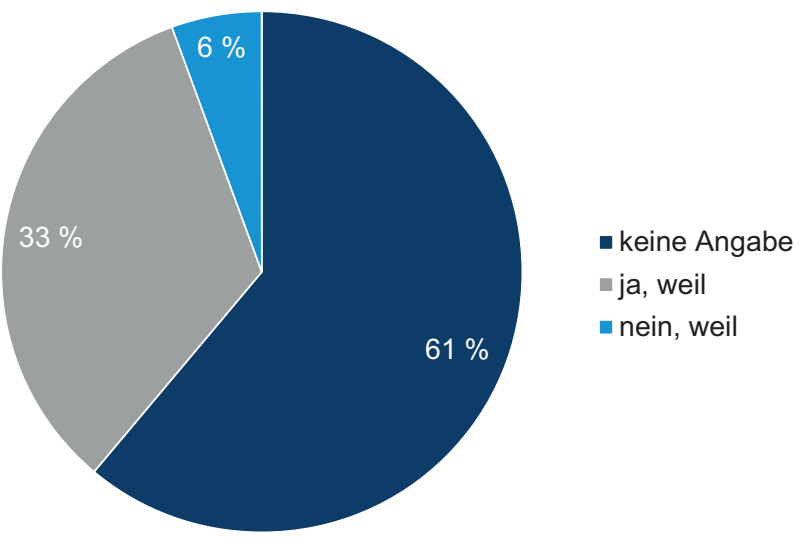

Abbildung 8.32 Bewertung des Transparenzgesetzes in Hamburg als Blaupause

Tabelle 8.47 Bewertung nach Antworthäufigkeit

\begin{tabular}{l|c|c}
\hline Antworten & Prozent & Anzahl \\
\hline keine Angabe & $61 \%$ & 22 \\
\hline ja, weil & $33 \%$ & 12 \\
\hline nein, weil & $6 \%$ & 2 \\
\hline Summe &. & $\mathbf{3 6}$ \\
\hline
\end{tabular}


Tabelle 8.48 Bewertung nach Antwortreihenfolge

\begin{tabular}{l|c|c}
\hline Antworten & Prozent & Anzahl \\
\hline ja, weil & $33 \%$ & 12 \\
\hline nein, weil & $6 \%$ & 2 \\
\hline keine Angabe & $61 \%$ & 22 \\
\hline Summe &. & $\mathbf{3 6}$ \\
\hline
\end{tabular}

\section{Bewertung:}

Auf die Frage, inwieweit das Transparenzgesetz in Hamburg als Vorreiter oder Blaupause geeignet ist, machen $61 \%$ der Befragten keine Angaben. Dies ist die Frage mit dem höchsten Anteil von „keine Angabe“ in dieser Studie. Möglicherweise deutet dies darauf hin, dass einer Mehrheit das Hamburger Transparenzgesetz und -portal gar nicht bekannt ist.

Immerhin $33 \%$ der Befragten bejahen die Frage. Aus ihrer Sicht stellen die mit dem HmbTG gesammelten positiven und negativen Erfahrungen eine gute Richtschnur dar und man habe einen Standard, auf dem man aufsetzen könne. Außerdem wird positiv angemerkt, dass die Informationen proaktiv per Gesetz verfügbar gemacht werden und nicht der expliziten Anfrage der Bürger bedürfen. Für einige ist das HmbTG mangels Alternativen in Deutschland ein gutes Beispiel. Kritisch wird - trotz Einordnung in der Kategorie ,ja“ jedoch angemerkt, dass vollständige Daten und nachvollziehbar redigierte Verträge benötigt werden, eine benutzerfreundliche Bedienbarkeit des Portals, noch umfangreichere Transparenzgesetze sowie eine gesamthafte Steuerbarkeit/Regulierung ausgehend von Aufsichtsbehörden, die hierfür gestärkt werden müssten.

Nur $6 \%$ der Befragten sehen im HmbTG definitiv keine Blaupause. Als Begründungen werden angeführt, dass Veröffentlichungen oftmals geschwärzt, die Informationen schwierig aufzufinden und unvollständig seien. Dies ist im Kern übereinstimmend mit der Kritik, die im Zusammenhang mit den „,ja, weil“-Begründungen geäußert wird. Abbildung 8.32, Tabelle 8.47 und Tabelle 8.48 zeigen die Werte. 
Frage 36: Welche Projekte beziehungsweise vorbildhaften Umsetzungen (Best Practices) bezogen auf die Öffnung öffentlicher Einkaufsdaten kennen Sie oder sollten wir noch ansprechen?

\section{Bewertung:}

Auf die Frage (offene Texteingabe), welche Projekte beziehungsweise Best Practices bekannt sind, reichen die Vorschläge von Hinweisen zur Finanzierung und Förderung, über erste Umsetzungsbeispiele, Portale, Projekte und Initiativen bis hin zu Länderbeispielen. Damit handelt es sich um zahlreiche Einzelnennungen, die sehr divers sind (siehe Tabelle 8.49). Demnach gibt es auch hier noch keine etablierten Standards, was einen Handlungsspielraum für alle Akteure eröffnet.

Tabelle 8.49 Vorgeschlagene Best Practices

\begin{tabular}{l|l}
\hline Kategorie & Best Practices \\
\hline Finanzierung/Förderung & - EU Fördermittelvorgaben \\
\hline Portale & - http://open.nrw.de \\
& - https://prozorro.gov.ua/ \\
& - https://dozorro.org/ \\
& - https://opendata.bonn.de/dataset/ausschreibungen- \\
& vergabeverfahren \\
& - Landesweit zentrales Vergabeportal des Landes Georgien \\
\hline Projekte/Initiativen & - http://www.contracting5.org \\
& - https://opentender.eu/ (Teil des DIGIWHIST-Projekts) \\
& - ICG (Institut für Corporate Governance) Initiative zur \\
& „Good Governance“ \\
\hline Länderbeispiele & - Großbritannien, Kanada, Ukraine, Paraguay, Kolumbien, \\
& Australien, Portugal, Finnland \\
\hline Standards & - https://standard.open-contracting.org \\
\hline Anwendungen & - Ausgabenanalyse \\
\hline
\end{tabular}


Frage 37: Welchen Zeithorizont halten Sie für die Öffnung des öffentlichen Einkaufswesens in Deutschland für realistisch?

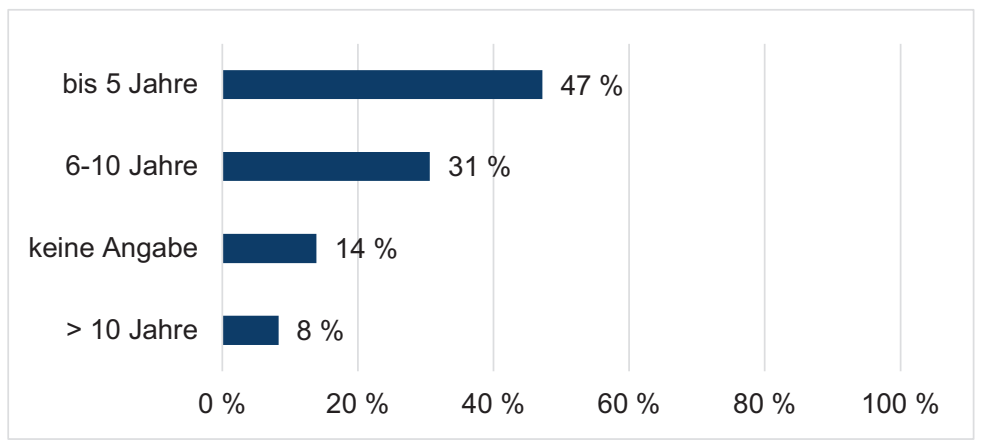

Abbildung 8.33 Geschätzter Zeithorizont zur Öffnung des öffentlichen Einkaufswesens

Tabelle 8.50 Geschätzter Zeithorizont nach Antworthäufigkeit

\begin{tabular}{l|c|c}
\hline Antworten & Prozent & Anzahl \\
\hline Bis 5 Jahre & $47 \%$ & 17 \\
\hline 6-10 Jahre & $31 \%$ & 11 \\
\hline keine Angabe & $14 \%$ & 5 \\
\hline 10 Jahre & $8 \%$ & 3 \\
\hline Summe & $\mathbf{1 0 0 \%}$ & $\mathbf{3 6}$ \\
\hline
\end{tabular}

Tabelle 8.51 Geschätzter Zeithorizont nach Antwortreihenfolge

\begin{tabular}{l|c|c}
\hline Antworten & Prozent & Anzahl \\
\hline Bis 5 Jahre & $47 \%$ & 17 \\
\hline 6-10 Jahre & $31 \%$ & 11 \\
\hline$>$ 10 Jahre & $8 \%$ & 3 \\
\hline keine Angabe & $14 \%$ & 5 \\
\hline Summe & $\mathbf{1 0 0 \%}$ & $\mathbf{3 6}$ \\
\hline
\end{tabular}




\section{Bewertung:}

Auf die Frage, welchen Zeithorizont die Befragten für die Öffnung des öffentlichen Einkaufswesens in Deutschland für realistisch halten, antworten nur $8 \%$ der Befragten, dass sie einen Horizont von $>10$ Jahren als realistisch einstufen. $31 \%$ schätzen einen Zeitraum von 6-10 Jahren als umsetzbar ein. Knapp die Hälfte der Befragten hält die Öffnung der öffentlichen Einkaufsdaten innerhalb von 5 Jahren für möglich $(47 \%)$. Abbildung 8.33 , Tabelle 8.50 und Tabelle 8.51 führen die entsprechenden Werte auf.

\subsubsection{Zusammenhangsanalysen und Vergleiche}

Wie unterscheiden sich die Werte der Zustimmung zwischen ,heutiger Veröffentlichung“ und unterstützter ,zukünftiger Veröffentlichung“ öffentlicher Einkaufsdaten?

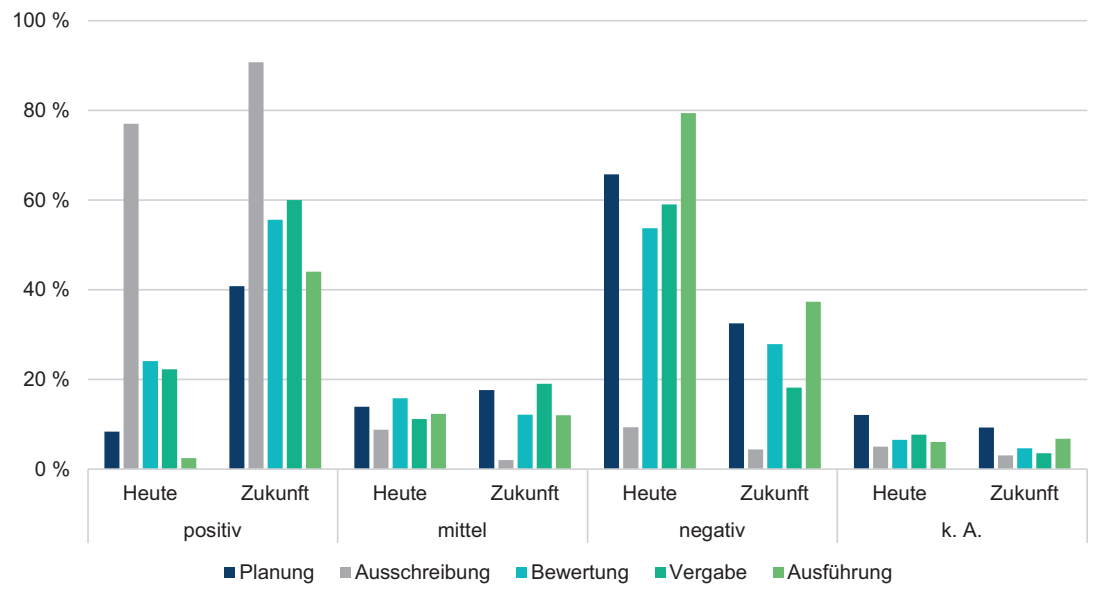

Abbildung 8.34 Vergleich Mittelwerte heutiger und künftiger Veröffentlichung öffentlicher Einkaufsdaten 
Tabelle 8.52 Vergleich Mittelwerte heutiger und künftiger Veröffentlichung öffentlicher Einkaufsdaten

\begin{tabular}{l|c|c|c|c|c|c|c|c}
\hline \multirow{2}{*}{ Antworten } & \multicolumn{2}{|c|}{ Positiv } & \multicolumn{2}{c|}{ Mittel } & \multicolumn{2}{c|}{ Negativ } & \multicolumn{2}{c}{ k. A. } \\
\cline { 2 - 10 } & Heute & Zukunft & Heute & Zukunft & Heute & Zukunft & Heute & Zukunft \\
\hline Planung & $8 \%$ & $41 \%$ & $14 \%$ & $18 \%$ & $66 \%$ & $32 \%$ & $12 \%$ & $9 \%$ \\
\hline Ausschreibung & $77 \%$ & $91 \%$ & $9 \%$ & $2 \%$ & $9 \%$ & $4 \%$ & $5 \%$ & $3 \%$ \\
\hline Bewertung & $24 \%$ & $56 \%$ & $16 \%$ & $12 \%$ & $54 \%$ & $28 \%$ & $7 \%$ & $5 \%$ \\
\hline Vergabe & $22 \%$ & $60 \%$ & $11 \%$ & $19 \%$ & $59 \%$ & $18 \%$ & $8 \%$ & $4 \%$ \\
\hline Ausführung & $2 \%$ & $44 \%$ & $12 \%$ & $12 \%$ & $79 \%$ & $37 \%$ & $6 \%$ & $7 \%$ \\
\hline
\end{tabular}

\section{Bewertung:}

Um zu verstehen, wie sich das Bild zwischen ,heutiger Veröffentlichung öffentlicher Einkaufsdaten“ (Frage 6) und ,künftiger Veröffentlichung öffentlicher Einkaufsdaten“ (Frage 13) verändert hat, ist ein Vergleich hilfreich. Dieser basiert auf den Mittelwerten der jeweiligen Teilprozesse in den Kategorien ,positiv“ (stimme einer Veröffentlichung zu), „mittel“ (unentschlossen) und ,negativ“ (stimme einer Veröffentlichung nicht zu). Die Ergebnisse sind in Abbildung 8.34 und Tabelle 8.52 dargestellt.

Mit Bezug auf den Teilprozess der Planung wächst die Zustimmung zu einer Veröffentlichung von Daten deutlich (von $8 \%$ im Mittel auf $41 \%$ im Mittel, dies bedeutet eine Steigerung um etwa das Fünffache). Gleichzeitig sinkt der Wert für eine entsprechende Ablehnung von im Mittel $66 \%$ auf $32 \%$. Mit Blick auf den Teilprozess der Ausschreibung hat sich die Zustimmung von im Mittel $77 \%$ auf im Mittel $91 \%$ sogar noch weiter erhöht. Die Teilprozesse Bewertung und Vergabe liegen laut den Befragten bei heute $24 \%$ respektive $22 \%$ Zustimmung - künftig befürworten dies $56 \%$ respektive $60 \%$. Auch hier ist eine deutliche Steigerung erkennbar (um mehr als das Doppelte). Der Teilprozess der Ausführung erfährt die deutlichste Zunahme in der Zustimmung von heute $2 \%$ im Mittel auf künftig $44 \%$ im Mittel - dies entspricht einer Zunahme um das 22-Fache.

Es wird deutlich, dass die Befragten eine stärkere Öffnung der öffentlichen Einkaufsdaten sämtlicher Teilprozesse unterstützen. 


\subsection{Zwischenfazit: Unterstützung einer differenzierten Öffnung öffentlicher Einkaufsdaten}

\subsubsection{Zentrale Einschätzungen zur Ausgangssituation}

Der Teilnehmerkreis dieser Studie bestand überwiegend aus fachkundigen Führungskräften und Experten, von denen sich fast alle (87\%) bereits moderat bis sehr stark mit offenen Verwaltungsdaten befassen. Konkreter nachgefragt, wie sich dies mit Blick auf öffentliche Einkaufsdaten verhält, antwortet gut die Hälfte, dass sie bereits Ansätze, Initiativen oder Programme nutze, um eine Öffnung dieser Daten zu fördern. Dies scheint damit zu tun zu haben, dass die Mehrheit hierin positive Effekte für ihre Organisation sieht. Nur eine Minderheit von $8 \%$ der Befragten sieht keinen positiven Effekt in der Öffnung öffentlicher Einkaufsdaten für ihre Organisation.

Die Prozessphasen Planung, Bewertung, Vergabe und Ausführung als Bestandteil des öffentlichen Gesamtbeschaffungsprozesses sind heute für Dritte überwiegend nicht zugänglich. Die meiste Zustimmung mit im Mittel $77 \%$ erfährt erwartungsgemäß der Teilprozess der Ausschreibung. Dieses Ergebnis geht konform mit der Portalanalyse in Abschnitt 7.3.9.

Obschon ein Großteil der öffentlichen Einkaufsdaten digital vorliegt, wird nur ein gutes Drittel der Daten heute online und maschinenlesbar bereitgestellt. Mit Blick auf eine künftige Öffnung der Daten müsste dieser Anteil ausgebaut werden. Dies entspricht ebenfalls der durchgeführten Portalanalyse (siehe Abschnitt 7.3.9), in welcher die häufigste Form die Bereitstellung der Daten in PDF-Dokumenten war.

Die Studie bestätigt ebenfalls die Resultate der Portalanalyse in Bezug auf die starke Fragmentierung der Portale und Plattformen, über die öffentliche Einkaufsdaten aktuell zugänglich gemacht werden: Es gibt kein überwiegend genutztes oder zentrales Portal. Vergleichbar fallen die Antworten auf die Frage nach den bekannten Anwendungen, Berichten und Visualisierungen, die auf öffentlichen Einkaufsdaten beruhen, aus. Ein gutes Viertel aller Nennungen (27\%) entfällt auf einmalige Angaben, was ebenfalls eine recht fragmentierte Anbieter- und Produktlandschaft widerspiegelt. Dieser Markt bietet demzufolge noch Geschäftsentwicklungspotential.

Die drei meistgenannten Hindernisse zur Öffnung der öffentlichen Einkaufsdaten sind die Vertraulichkeit der Informationen, der Datenschutz sowie das bestehende Vergaberecht. Wie bereits in den vorhergehenden Kapiteln diskutiert, sind hier sowohl Aufklärung als auch die Einbindung der Anforderungen in die 
jeweiligen Verwaltungsprozesse vonnöten sowie Schärfungen der Definitionen vorzunehmen.

Bei den nötigen Voraussetzungen wird der politische Wille zur Schaffung von Transparenz von überraschend vielen Befragten - nämlich von $92 \%$ - als hoch eingeschätzt. Das bedeutet, dass nahezu alle Befragten dieser Studie der Ansicht sind, dass der politische Wille für eine Öffnung öffentlicher Einkaufsdaten eine Grundvoraussetzung ist, die momentan zu fehlen scheint. Mit erheblichem Abstand folgt mit $47 \%$ die Forderung nach einer Veränderung der Rechtslage. An dritter Stelle steht die Einigkeit über Grenzen/Möglichkeiten der Öffnung. Auch dies bestätigt, dass es nicht alleine die bestehende Rechtslage oder gar eine unausgereifte Technologie sind, die eine Öffnung verhindern. Vielmehr scheint es an der Kommunikation, an dem Dialog, über gemeinsame Ziele zu mangeln.

\subsubsection{Erwartete Entwicklungen}

Der Vergleich der Antworten auf die Frage: „Wer hat heute Zugriff auf öffentliche Einkaufsdaten?“, und die Frage: „Wer sollte künftig Zugriff hierauf haben?“, zeigt, dass sich die Befragten eindeutig für eine weitere Öffnung der öffentlichen Einkaufsdaten aussprechen, und zwar durchaus über alle Teilprozesse hinweg, jedoch in unterschiedlichem Maße. Mit einer Zunahme um das 22-Fache ist der Teilprozess der Ausführung Spitzenreiter, mit einer Zunahme um mindestens das Doppelte folgen die Teilprozesse der Bewertung und Vergabe. Uneinigkeit herrscht beim Teilprozess der Planung - Zustimmung und Ablehnung befinden sich hier auf ähnlichem Niveau (trotz Steigerung der Zustimmung um das 5-Fache), was größeren Diskussionsbedarf andeutet.

Wenn es darum geht, wer Zugriff auf welche Teilprozesse haben sollte, zeichnet sich ab, dass in der Sicht der Befragten nicht alle Akteure gleichermaßen Zugriff auf alle öffentlichen Einkaufsdaten haben sollten - eine Differenzierung ist gewünscht, möglicherweise in Abhängigkeit von der vorhandenen und möglichen Kompetenz, die die entsprechenden Akteure einbringen können. So befürworten fast drei Viertel, dass die Verwaltungen Zugriff auf alle Teilprozesse haben sollten. Das ist nicht überraschend. Schaut man auf die anderen Akteure und die einzelnen Teilprozesse, so fällt auf, dass Wirtschaft und Öffentlichkeit in erster Linie Zugriff auf Daten der Ausschreibung erhalten sollen, gefolgt von der Planung und Vergabe. Die Teilprozesse Ausführung und Bewertung stehen hier weniger im Fokus. Die Wissenschaft steht insgesamt an letzter Stelle, man räumt ihr lediglich noch vor Wirtschaft und Öffentlichkeit Zugriff auf die Teilprozesse der Bewertung und Ausführung ein. 
Die Mehrheit der Teilnehmer wünscht sich eine gewisse Selektion der zu veröffentlichenden Einkaufsdaten. Ersichtlich wird das daran, dass sich nur ein Drittel für die Offenlegung der Daten aller Projekte und der Daten unterhalb eines bestimmten Volumens ausspricht. Gleichzeitig wird von $75 \%$ der Teilnehmer aber die Offenlegung der Daten von Projekten mit hoher beziehungsweiser negativer Außenwirkung befürwortet. In Bezug auf die Einschränkung mit Blick auf bestimmte Warengruppen oder Sektoren ist Diskussionsbedarf zu vermuten, da dies von etwa der gleichen Zahl der Teilnehmer verneint als auch bejaht wird (vor allem hinsichtlich Sicherheit und Verteidigung).

Die Mehrheit der Befragten spricht sich für eine künftige Bereitstellung offener öffentlicher Einkaufsdaten über ein zentrales, einheitliches Portal aus. Dieser Wunsch ist vor dem Hintergrund der derzeit bestehenden stark fragmentierten Portal- und Anbieterlandschaft nachvollziehbar.

Wirtschaftliche Konsequenzen der Bereitstellung offener öffentlicher Einkaufsdaten über den govdata.de-Verbund sind laut der Mehrheit der Befragten die Zeitersparnis für Recherchen öffentlicher Ausschreibungen, die Kooperation über Vergabestellen hinweg sowie die Erhöhung der Datenqualität. Interessant und unerwartet ist, dass nur $14 \%$ eine Erhöhung des Verwaltungsaufwands mit der Öffnung der öffentlichen Einkaufsdaten verbinden.

Als wesentlicher Impuls wird die Auswertung der Daten gesehen, gefolgt von der Förderung innovativer Beschaffungslösungen sowie der Verknüpfung öffentlicher Einkaufsdaten mit anderen Daten. Dies findet sich auch in den Antworten auf die Fragen nach sinnvollen Anwendungen, Berichten oder Visualisierungen sowie Lösungen und Geschäftsmodelle wieder. Die Nennungen signalisieren einerseits den Bedarf und verweisen andererseits auf die Kreativität und das (unternehmerische) Potential im Umfeld öffentlicher Einkaufsdaten. Der Bedarf nach Statistiken, Auswertungsmöglichkeiten und sogar der Verknüpfung öffentlicher Einkaufsdaten mit anderen Daten ist vorhanden, ebenso wie Ansätze, dieses Potential zu heben. Abschließend wird für alle Akteure ein deutlicher Nutzen konstatiert - an erster Stelle liegen Wirtschaft, Verwaltung und Wissenschaft. Politik und Medien liegen an zweiter Stelle, an letzter Stelle die Bürger und NGOs.

\subsubsection{Rand- und Rahmenbedingungen}

Auf die Frage nach Maßnahmen in der Beteiligung Dritter zur künftigen Verbesserung der Legitimation von Einkaufsentscheidungen erfahren die Einbeziehung neutraler Dritter in den Auswahlprozess, das Engagement in frühen Phasen sowie externes Vertrags- und Leistungsmonitoring am meisten Zuspruch. 
Um das Engagement verschiedener Beteiligter zu fördern, spricht sich eine gute Hälfte für offene Formate aus. Dies entspricht den dargestellten Ergebnissen aus der Literatur in Abschnitt 6.5.1, Abschnitt 6.5.3 und Abschnitt 7.4.3.

Etwa zwei Drittel der Befragten stimmen zu, dass durch die Öffnung der öffentlichen Einkaufsdaten Korruption am ehesten bei der Ausschreibungsbekanntmachung, der Vergabe und der Projektkontrolle reduziert werden kann. Die geringsten Möglichkeiten werden bei Planung, Projektdurchführung sowie Rechnungs- und Zahlungsabwicklung gesehen. Hier wäre es interessant, wissenschaftliche Erhebungen zu nutzen, um diese Zusammenhänge zu erforschen.

Als Maßnahmen, Korruption nachhaltig zu reduzieren, werden in erster Linie die Durchführung regelmäßiger interner und externer Auftragskontrollen sowie die Einführung eines zentralen Korruptionsregisters bestätigt. Außerdem befürwortet eine Mehrheit die Nutzung öffentlicher Vergabeverfahren sowie eine Schwarzliste zu Lieferanten. Demnach sehen die Experten insbesondere in der Nutzung Dritter beziehungsweise der Öffentlichkeit einen Mehrwert, was durch die Literatur untermauert wird.

In prinzipieller Übereinstimmung konstatieren die Befragten, es habe „keine wesentlichen Änderungen“ infolge der Vergaberechtsreform gegeben. Doch wird positiv angemerkt, dass mit der eVergabe nun Daten im Oberschwellenbereich verbindlich bereitstehen und so eine gute Basis für eine elektronische Auswertung und Strukturierung bilden. Hieran wird die Erwartung geknüpft, dass damit bald für den Unterschwellenbereich ähnlich gehandelt wird. Künftige Vergaberechtsreformen sollten vor allem die gesetzliche Verankerung der Datenöffnung entlang des gesamten Einkaufsprozesses und im Unterschwellenbereich beinhalten. IFG und IWG, aber auch der Datenschutz, sollten weiter präzisiert und verschärft werden.

Die Vorschläge zu Best Practices bestehen aus 13 Einzelnennungen bei 72 Antwortoptionen (zwei optionale Freitextfelder pro Teilnehmer), was einer Rücklaufquote von $18 \%$ entspricht. Die niedrige Quote dürfte darauf zurückzuführen sein, dass es noch keine allgemein anerkannten vorbildhaften Umsetzungen in diesem Bereich gibt. Diese sollten somit noch stärker gefördert, verankert und kommuniziert werden, vor allem vor dem Hintergrund, dass sich mehr als zwei Drittel der Teilnehmer eine Umsetzung dieses Themas in den nächsten zehn Jahren vorstellen können.

Dass es auf internationaler Ebene durchaus Best Practices gibt, wird das nächste Kapitel aufzeigen. So gibt es diverse Lösungsansätze, mit Blick auf Europa zum Beispiel in der Slowakei und in Frankreich. Hier werden Vorgehensweisen erprobt, die für Deutschland wichtige Impulse geben könnten. Gleiches gilt für die drei zukunftsweisenden Technologie-Beispiele, aber auch alle anderen gelisteten Best Practices. 
Open Access Dieses Kapitel wird unter der Creative Commons Namensnennung 4.0 International Lizenz (http://creativecommons.org/licenses/by/4.0/deed.de) veröffentlicht, welche die Nutzung, Vervielfältigung, Bearbeitung, Verbreitung und Wiedergabe in jeglichem Medium und Format erlaubt, sofern Sie den/die ursprünglichen Autor(en) und die Quelle ordnungsgemäß nennen, einen Link zur Creative Commons Lizenz beifügen und angeben, ob Änderungen vorgenommen wurden.

Die in diesem Kapitel enthaltenen Bilder und sonstiges Drittmaterial unterliegen ebenfalls der genannten Creative Commons Lizenz, sofern sich aus der Abbildungslegende nichts anderes ergibt. Sofern das betreffende Material nicht unter der genannten Creative Commons Lizenz steht und die betreffende Handlung nicht nach gesetzlichen Vorschriften erlaubt ist, ist für die oben aufgeführten Weiterverwendungen des Materials die Einwilligung des jeweiligen Rechteinhabers einzuholen.

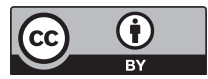

